\title{
What is the prevalence of musculoskeletal problems in the elderly population in developed countries? A systematic critical literature review
}

\author{
René Fejer ${ }^{1 *}$ and Alexander Ruhe ${ }^{2}$
}

\begin{abstract}
Background: The proportion of older people will be tripled by the year 2050. In addition, the incidence of chronic musculoskeletal (MSK) conditions will also increase among the elderly people. Thus, in order to prepare for future health care demands, the magnitude and impact of MSK conditions from this growing population is needed. The objective of this literature review is to determine the current prevalence of MSK disorders in the elderly population.

Methods: A systematic literature search was conducted in Pubmed on articles in English, published between January 2000 and July 2011. Studies from developed countries with prevalence estimates on elderly people (60+) on the following MSK conditions were included: Non-specific extremity pain, rheumatoid arthritis, osteoarthritis, osteoporosis, and back pain. The included articles were extracted for information and assessed for risk of bias.

Results: A total of 85 articles were included with 173 different prevalence estimates. Musculoskeletal disorders are common in the elderly population, but due to heterogeneity of the studies, no general estimate on the prevalence of MSK can be determined. Women report more often MSK pain than men. Overall, prevalence estimates either remain fairly constant or increase slightly with increasing age, but with a tendency to decrease in the oldest (80+) people.

Conclusions: Musculoskeletal disorders remain prevalent in the elderly population. Given the increasing proportion of elderly population in the world population and the burden of MSK diseases among the elderly people, efforts must be made to maintain their functional capacity for as long as possible through optimal primary and secondary health care.
\end{abstract}

Keywords: Systematic literature review, Musculoskeletal disease, Elderly population, Osteoarthritis, Rheumatoid arthritis, Osteoporosis, Back pain

\section{Background}

According to the United Nations (UN), the proportion of older people (i.e. aged 60 and over) will triple over the next 40 years and will account for more than $20 \%$ of the world's population by year 2050 [1]. In addition, it is estimated that one in five of the elderly population will be more than 80 years old in 2050. The exponential increase of elderly people is mainly due to a rise in life expectancy, especially in the developing countries. Along with the rise in the life expectancy there is also a rise in

\footnotetext{
* Correspondence: rene.fejer@slb.regionsyddanmark.dk

${ }^{1}$ The Research Department, the Spine Centre of Southern Denmark, Hospital Lillebaelt, Middelfart, Denmark

Full list of author information is available at the end of the article
}

the incidence of non-communicable chronic conditions which again leads to increasing morbidity and disability [2]. According to the World Health Organization (WHO), one of the major disabling conditions among the elderly population is musculoskeletal (MSK) disorders $[3,4]$. The WHO has specifically identified four major disabling MSK conditions: osteoarthritis (OA), rheumatoid arthritis (RA), osteoporosis (OP), and back pain (BP) [4].

In 1998, the Bone and Joint Decade (BJD) 2000-2010 collaboration was initiated and endorsed by the UN and WHO, with the overall goal to reduce the burden and cost of MSK diseases [5,6]. In 2003, the WHO's Global Burden of Disease study and the Bone and Joint

\section{Biomed Central}


Monitoring Project conducted a large report on the burden of MSK disorders through the existing data on the four major MSK conditions (OA, RA, OP, and low back pain (LBP)) $[4,5]$. From this report, it is clear that the burden of these major MSK conditions increases with age.

From a health care perspective, the rising proportion and burden of older people demands that health care professionals increase their awareness of the health and disability of this particular population. Accordingly, there is a need to better understand the current magnitude and impact of MSK conditions from this growing population.

The aim of this paper is to estimate the current prevalence of musculoskeletal disorders in the elderly population by conducting a systematic literature review. Specifically, the objective was to estimate the prevalence of non-specific musculoskeletal pain, OA, RA, OP, and BP among older people in developed countries. Any methodological shortcomings will be discussed and future recommendations will be provided.

\section{Methods \\ Definitions}

Musculoskeletal pain in this review refers to the following five overall conditions: 1) non-specific MSK pain in the extremities, 2) RA, 3) OA, 4) OP (either spine or hip or a combination of both), and 5) BP (i.e. neck pain (NP), mid back pain (MBP), and LBP). The older population is defined as people aged 60 and over according to the UN's cut-off criterion [1]. The term "magnitude" in this review refers to the relative size (i.e. prevalence) of the selected MSK conditions. Hence, the quality of life, cost-of-illness, or social/personal burden of MSK disorders is not included. Developed countries are defined as countries with an advanced economy according to the International Monetary Fund, which includes 35 countries (Additional file 1) [7].

\section{Search design}

A systematic literature search was conducted in Pubmed (www.pubmed.org) and included studies published between January $1^{\text {st }} 2000$ and July $1^{\text {st }} 2011$. The timeperiod was chosen in order to only include studies published after the WHO reports [3,4]. Search terms included both free text and MeSH terms and were combined by Boolean terms (AND, OR, NOT) (Additional file 2). The following main terms were included: "musculoskeletal", "rheumatoid arthritis", "osteoarthritis", and "osteoporosis". The MeSH terms were limited to only include studies containing "epidemiology", "etiology", or "diagnosis". These were again combined with "prevalence", "cross-sectional studies". The search was limited by type of papers (review, government publications, technical reports or journal articles), age (MeSH terms: "aged" and "aged, 60 and over") and finally restricted to English language only. No additional search was conducted. The retrieval of potentially relevant articles was conducted in two phases by one examiner. The first phase focused on identifying relevant studies through

Table 1 Inclusion and exclusion criteria

\begin{tabular}{|c|c|}
\hline Inclusion & Exclusion \\
\hline $\begin{array}{l}\text { - Original observational studies or reports; primarily cross-sectional } \\
\text { and cohort studies }\end{array}$ & $\begin{array}{l}\text { - If more than one article presenting results from the same study existed } \\
\text { then only the most relevant article was included. }\end{array}$ \\
\hline - Studies reporting results specifically on people aged 60 and over & $\begin{array}{l}\text { - No reviews, experimental or clinical trials, or studies with subsample of } \\
\text { the original study sample, unless it is still a representative sample and } \\
\text { reports new relevant information }\end{array}$ \\
\hline $\begin{array}{l}\text { - Representative of the general population (study samples from } \\
\text { nursing homes, etc. are accepted) }\end{array}$ & - No working populations \\
\hline $\begin{array}{l}\text { - Only following musculoskeletal (MSK) conditions: } \\
\text { 1)Non-specific extremity MSK } \\
\text { 2)Back pain (+ divided by region) } \\
\text { 3)Osteoarthritis in larger joints of the extremities (i.e. shoulder, } \\
\text { elbow, hand/wrist, hip, knee, ankle/foot) } \\
\text { 4) Rheumatoid arthritis } \\
\text { 5) Osteoporosis }\end{array}$ & - No native/aboriginal populations \\
\hline $\begin{array}{l}\text { - Studies from developed countries only (e.g. countries with } \\
\text { "advanced economies" according to IMF) }\end{array}$ & - Studies reporting general MSK pain with no specific anatomical area \\
\hline - Any type of prevalence/incidence & - No traumatic related injuries \\
\hline $\begin{array}{l}\text { - Prevalence/incidence estimates specifically on people aged } \\
60 \text { and over }\end{array}$ & - No secondary MSK conditions (i.e. osteoporotic fractures) \\
\hline $\begin{array}{l}\text { - In studies with results from more than one period/survey, } \\
\text { only the latest }\end{array}$ & $\begin{array}{l}\text { - No combined anatomical sites (e.g. neck + shoulder pain), except for back } \\
\text { pain which is usually low back pain. }\end{array}$ \\
\hline \multirow[t]{2}{*}{ - year was included } & - No OA in minor joints (such as in a single phalanx joint, facet joints, etc.) \\
\hline & - Indirect/weighted/adjusted prevalence estimates. \\
\hline
\end{tabular}


the title and abstract. This was followed by retrieval of all full-text articles for further eligibility. As Pubmed adds papers or change $\mathrm{MeSH}$ terms retrospectively, the search was repeated after July $1^{\text {st }}$. The last search was conducted September $1^{\text {st }}$ 2011. No additional searches were conducted, nor were any authors contacted.

\section{Eligibility criteria}

Only observational studies from developed countries that reported specific MSK disorders on older people aged 60 and over were included. Thus, studies reporting general MSK pain were excluded. Preferably, the study sample had to represent the general population, but as some individuals may live in nursing homes etc., such studies were also accepted. Table 1 lists the full inclusion and exclusion criteria used in this literature review.

\section{Extraction of information}

All core information from the included studies was extracted by an unblinded examiner. The most relevant information were: Article details, study objective(s), study design, method of data collection, sampling method and sample data, disease definition, and outcome data (Table 2). If the included study referred to another reference (i.e. another paper, report, or website) for a more detailed description of the study cohort, then that reference was perused for additional information if it was accessible.

\section{Risk of bias assessment}

The quality of each study was determined by assessing the risk of bias [8]. Recently, Viswanathan et al. have identified 29 practical and validated items that may be used to evaluate the risk of bias and precision of observational studies [9]. This bank of items covers a range of different study designs and the authors have provided instructions as to what items to use depending on the studies under assessment. Thus, only items related to our main objectives were identified and criteria for each

\section{Table 2 List of items extracted from each article}

\begin{tabular}{ll}
\hline 1. & Article details (author(s), title, country, source) \\
2. & Objective(s) of study \\
3. & Study design (cross-sectional or cohort/longitudinal) \\
4. & Method of data collection (registry, questionnaire, interview, \\
5. & Sampling method and sample data (age, gender ratio, target \\
6. & Description of MSK condition (definition, type and validation \\
7. & Outcome data (type of prevalence/incidence, results \\
8. & Own remaing
\end{tabular}

item were defined to fit our main objective (Table 3). The layout of the questionnaire was slightly modified for practical reasons, but no other changes were made. The chosen items focused on selection bias, information bias, and the overall interpretation of each study. Relevant criteria to assist in determining the risk of bias in a study were specified to each item. No validation of the included items was performed.

\section{Data analysis}

The extracted data was presented in separate tables for each of the included MSK conditions. In studies where the results were only presented graphically, best effort was made to determine the prevalence estimates from the graphs (without decimals). Both total and gender prevalence estimates as well as age related changes were reported when possible. In addition, the attempt was made to present pooled means of prevalence estimates on fairly homogeneous studies.

\section{Results \\ Search results}

In total, 5097 articles were found through the search strategy (Figure 1). Based on either their title or abstract, 185 were subsequently retrieved and reviewed. Of these, 100 articles were rejected, mainly because prevalence estimates on elderly aged 60 and over was not reported or could not be determined (82\%) (Additional file 3). Other reasons for exclusions were 1 ) the studies did not fulfil the inclusion/exclusion criteria (14\%) and 2) articles reporting results that were already published in other articles (i.e. duplicate publications) (4\%). Thus, in all 85 articles were included in this review.

\section{Study characteristics}

The included articles were published in 39 different journals of which 4 journals (Spine (26\%), Rheumatology (18\%), Annals of Rheumatic Diseases (18\%), Arthritis \& Rheumatism (15\%)) accounted for approximately three quarters of all journals. There was an uneven distribution of publications between 2000 and 2011, but with no clear patterns across the decade. The majority of the studies were from Europe (58\%) followed by Australasia (21\%), North America (18\%) and Middle East (4\%).

\section{Risk of bias within each study and across studies}

Overall, $25 \%$ of the studies were determined as having a low risk of bias and $11 \%$ were deemed as having a high bias risk (Figure 2 and Additional file 4). Thus, in approximately $65 \%$ of the studies it was unclear if risk of bias were either low or high, mainly because it was difficult to determine if the final study sample was truly representative of the target population. The risk of bias for 
Table 3 Items chosen to assess risk of bias of the included studies

\begin{tabular}{|c|c|c|c|c|}
\hline $\begin{array}{l}\text { Item number } \\
\text { from original } \\
\text { study* }\end{array}$ & $\begin{array}{l}\text { Dimension } \\
\text { of bias }\end{array}$ & $\begin{array}{l}\text { Methods } \\
\text { domain }\end{array}$ & $\begin{array}{l}\text { Assessment } \\
\text { question }\end{array}$ & Criteria / definitions / categories \\
\hline \multirow[t]{6}{*}{ Q2 } & \multirow{6}{*}{$\begin{array}{l}\text { Selection } \\
\text { bias }\end{array}$} & \multirow{6}{*}{$\begin{array}{l}\text { Sample } \\
\text { definition and } \\
\text { selection }\end{array}$} & \multirow{6}{*}{$\begin{array}{l}\text { - Are critical inclusion/exclusion criteria } \\
\text { clearly stated? }\end{array}$} & - Target population described? \\
\hline & & & & $\begin{array}{l}\text { - Ascertainment procedure for target sample } \\
\text { described? }\end{array}$ \\
\hline & & & & $\begin{array}{l}\text { - Study sample representative of the target } \\
\text { population described? }\end{array}$ \\
\hline & & & & - Age range, gender, etc. described? \\
\hline & & & & - Specific inclusion/exclusion criteria stated? \\
\hline & & & & - Sample size described? \\
\hline \multirow[t]{5}{*}{ Q3 } & \multirow[t]{5}{*}{$\begin{array}{l}\text { Information } \\
\text { bias }\end{array}$} & \multirow{5}{*}{$\begin{array}{l}\text { Sample } \\
\text { definition and } \\
\text { selection }\end{array}$} & \multirow{5}{*}{$\begin{array}{l}\text { - Are the inclusion/exclusion criteria } \\
\text { measured using valid and reliable } \\
\text { measures }\end{array}$} & $\begin{array}{l}\text { - Ascertainment procedure: Random, stratified, } \\
\text { cluster, etc. (if applicable) }\end{array}$ \\
\hline & & & & $\begin{array}{l}\text { - Registry (census, GP databases) (reporting bias?) } \\
\text { (if applicable) }\end{array}$ \\
\hline & & & & $\begin{array}{l}\text { - Medical records (clinical or hospital records) } \\
\text { (if applicable) }\end{array}$ \\
\hline & & & & $\begin{array}{l}\text { - Non-response analysis (non-response bias) } \\
\text { (if applicable) }\end{array}$ \\
\hline & & & & $\begin{array}{l}\text { - Sample size: is it justified or is a power calculation } \\
\text { provided? }\end{array}$ \\
\hline \multirow[t]{5}{*}{ Q14 } & \multirow[t]{5}{*}{$\begin{array}{l}\text { Information } \\
\text { bias }\end{array}$} & \multirow[t]{5}{*}{$\begin{array}{l}\text { Soundness of } \\
\text { information }\end{array}$} & \multirow[t]{5}{*}{$\begin{array}{l}\text { - Are outcome measures assessed using } \\
\text { valid and reliable measures? }\end{array}$} & $\begin{array}{l}\text { - Questionnaire (is it valid and/or reproducible?) } \\
\text { (if applicable) }\end{array}$ \\
\hline & & & & $\begin{array}{l}\text { - Registry (i.e. census, GP databases) (reporting bias?) } \\
\text { (if applicable) }\end{array}$ \\
\hline & & & & $\begin{array}{l}\text { - Interviewing bias (i.e. structured, semi-structured, } \\
\text { objective) (if applicable) }\end{array}$ \\
\hline & & & & $\begin{array}{l}\text { - Self-reporting (risk of recall bias; shorter recall better } \\
\text { than longer recall) (if applicable) }\end{array}$ \\
\hline & & & & $\begin{array}{l}\text { - Observation, examination procedure (observer bias?) } \\
\text { (if applicable) }\end{array}$ \\
\hline \multirow[t]{7}{*}{ • Q7 } & \multirow[t]{7}{*}{$\begin{array}{l}\text { - Performance } \\
\text { bias }\end{array}$} & \multirow[t]{7}{*}{ - Exposure } & \multirow[t]{4}{*}{$\begin{array}{l}\text { - What is the level of detail in describing } \\
\text { the outcome? }\end{array}$} & $\begin{array}{l}\text { - Definition of the MSK condition; anatomical, } \\
\text { physiological. (required) }\end{array}$ \\
\hline & & & & $\begin{array}{l}\text { - Definition of symptom(s) (pain, problem, other) } \\
\text { (required) }\end{array}$ \\
\hline & & & & $\begin{array}{l}\text { - Definition of period of symptom(s) (required, only } \\
\text { if applicable) }\end{array}$ \\
\hline & & & & $\begin{array}{l}\text { - Description of pain intensity (if applicable, not } \\
\text { required) }\end{array}$ \\
\hline & & & \multirow[t]{3}{*}{ - Overall judgment } & $\begin{array}{l}\text { - Low risk of bias: Bias, if present, is unlikely to alter the } \\
\text { results seriously }\end{array}$ \\
\hline & & & & $\begin{array}{l}\text { - Unclear risk of bias: Impossible to determine risk of } \\
\text { bias (either missing or not described well enough) }\end{array}$ \\
\hline & & & & - High risk of bias: Bias may alter the results seriously \\
\hline
\end{tabular}

* Viswanathan M, Berkman ND. Development of the RTI item bank on risk of bias and precision of observational studies. J Clin Epidemiol 2011, 65:163-178.

each of the included studies is presented within each of the musculoskeletal conditions.

\section{Prevalence of musculoskeletal disorders in the elderly population}

A total of 173 different prevalence estimates were extracted from the 85 included studies. The most commonly reported MSK condition (i.e. number of prevalence estimates) was BP (29\%), OA and OP (17\%), followed by RA (8\%), ankle/foot pain (8\%), knee pain (6\%), hip pain (5\%), shoulder pain (5\%), hand/wrist pain (3\%), and elbow pain (3\%).

\section{Prevalence of RA}

Rheumatoid arthritis was described in 12 studies with a total of 13 different point prevalence estimates [10-21] 


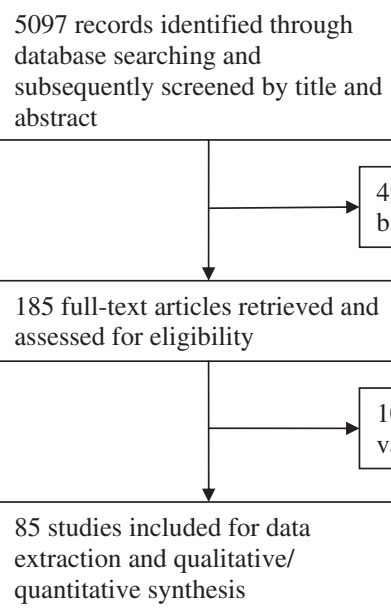

185 full-text articles retrieved and assessed for eligibility

85 studies included for data

extraction and qualitative/

quantitative synthesis

Figure 1 Flow chart of search results.

(Table 4). Seven (58\%) were of low risk of bias $[10,11,13,16,17,19,20]$ and only one study [18] was deemed as being of high risk of bias (Table 4 and Additional file 4).

The prevalence estimates that were based on clearly defined criteria (typically the 1987 American College of Rheumatology (ACR) criteria [22]) ranged between $0.4 \%$ and $2.2 \%$. The prevalence of RA was higher among women. No clear age related differences could be determined, but generally the prevalences were minimal across ages.

\section{Prevalence of $O A$}

Sixteen studies reported prevalence estimates on OA in four different anatomical sites (knee, hand, hip, and lumbar spine) either based on symptomatic findings only, radiographic findings only, or on a combination of both [11,18,23-36] (Table 5). Of these studies, five (31\%) were judged as being of low risk [11,23-25,30] and only one study (6\%) of high risk of bias [18] (Table 5 and Additional file 4).

\section{Lumbar spine $O A$}

Two Japanese studies on lumbar spine radiographic OA, using a higher Kellgren-Lawrence (K-L) grade $(\geq 3)$, reported point prevalences of $40 \%-75 \%$ in the $60-69$ year olds to $80 \%-90 \%$ in the $80+$ age group $[33,35]$.

\section{Hip $O A$}

Only three studies on hip OA were found in this review $[18,23,37]$, two studies on symptomatic hip OA $[18,37]$ and one on combined symptomatic/radiographic hip OA [23]. The self reported hip OA were about three times higher (17-22\%) than found through clinical examination (approx. 8\%) and more common in women than in men [23]. Combined symptomatic/radiographic hip OA increased from $2 \%$ in the $60-64$ year olds to $3 \%$ in the 75-79 year olds, but then decreased slightly in the $80+$ year olds.

\section{Knee OA}

Knee OA was reported in 11 studies [11,18,23,25,27,28,30-34] and presented 14 different prevalence estimates (Table 5). The ACR clinical criteria [38] for knee OA was used in two out of three studies on symptomatic knee pain and showed fairly similar prevalence estimates (28-33\%).

All studies on radiographic knee OA only (i.e. without reported pain) either used the K-L grade $2[39,40]$ or higher criteria for OA [27,28,31-34]. Nevertheless, great variations in point prevalence estimates were reported. For example, in women in their sixties, OA was present in $40 \%$ to $57 \%$, and in the seventies it ranged between $54 \%$ and $74 \%$. In men, larger differences were found (60s: $4 \%-35 \%)$ and (70s: $18 \%-51 \%)$. Overall, higher OA estimates were reported with increasing age.

For the combined knee $\mathrm{OA}$ and reported pain, generally larger gender differences were seen (Table 5) and more variation in age trends were also noted $[23,27,28,31,32]$. Painful knee OA increased with age until approximately at age $80+$ where a slight decrease was reported in two out of the four studies $[11,18,25,30]$.

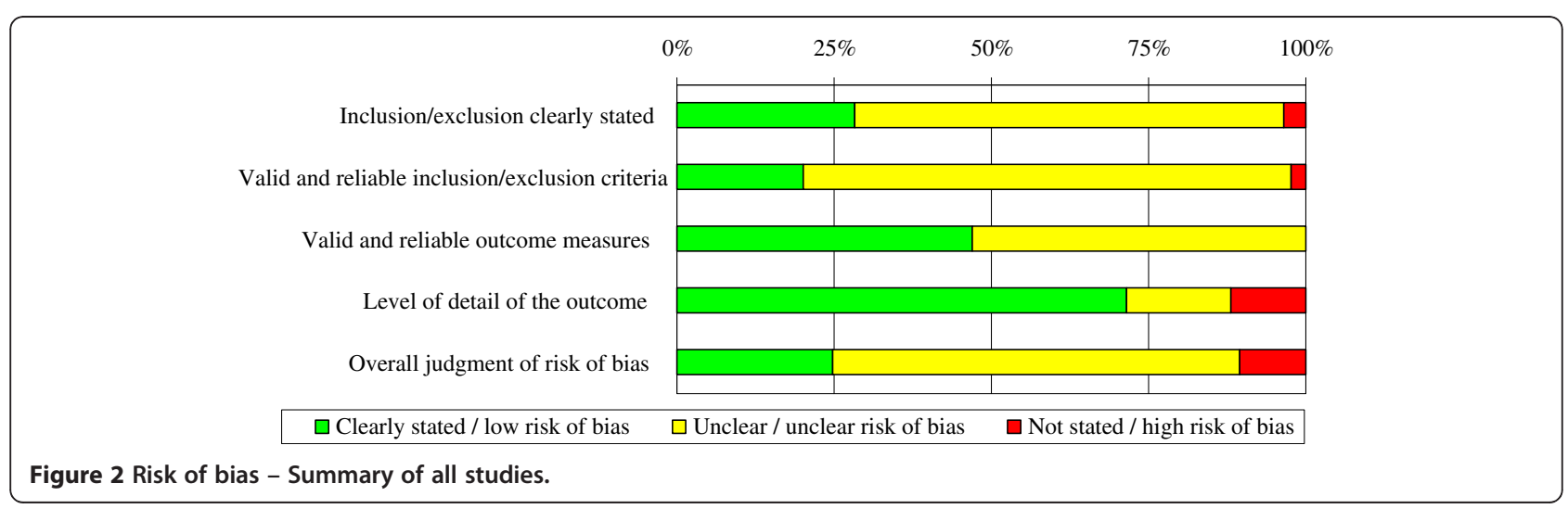


Table 4 Description of studies on rheumatoid arthritis (RA)

\begin{tabular}{|c|c|c|c|c|c|c|c|c|c|c|c|c|c|}
\hline \multirow{2}{*}{$\begin{array}{l}\text { First author } \\
\text { Publ. year } \\
\text { Country }\end{array}$} & \multirow{2}{*}{$\begin{array}{l}\text { Study design / } \\
\text { Population /Method } \\
\text { of collection }\end{array}$} & \multicolumn{3}{|c|}{ Sample size } & \multirow{2}{*}{$\begin{array}{l}\text { Crude } \\
\text { response } \\
\text { rate }(\%)\end{array}$} & \multirow{2}{*}{$\begin{array}{l}\text { Outcome } \\
\text { definition }\end{array}$} & \multirow{2}{*}{$\begin{array}{l}\text { Outcome } \\
\text { assessment } \\
\text { method }\end{array}$} & \multirow{2}{*}{$\begin{array}{l}\text { Prevalence } \\
\text { period }\end{array}$} & \multirow[t]{2}{*}{ Age } & \multicolumn{3}{|c|}{ Prevalence* $(95 \% \mathrm{Cl})$} & \multirow{2}{*}{$\begin{array}{l}\text { Risk } \\
\text { of } \\
\text { bias }\end{array}$} \\
\hline & & Total & $M$ & $F$ & & & & & & $M$ & $\mathrm{~F}$ & Total & \\
\hline \multirow{2}{*}{$\begin{array}{l}\text { Andrianakos [10] } \\
2006 \text { Greece }\end{array}$} & \multirow{2}{*}{$\begin{array}{l}\text { 1966-99, (19+ yo), the total adult } \\
\text { population in } 7 \text { mixed } \\
\text { communities + random sample } \\
\text { in another } 2 \text { mixed communities } \\
\text { (the ESORDIG study). Home visit } \\
\text { by a rheumatologist. Interview } \\
\text { and examination }\end{array}$} & \multirow[t]{2}{*}{8740} & \multirow[t]{2}{*}{4269} & \multirow[t]{2}{*}{4471} & \multirow[t]{2}{*}{82} & \multirow{2}{*}{$\begin{array}{l}\text { ACR } 1987 \\
\text { criteria }\end{array}$} & \multirow{2}{*}{$\begin{array}{l}\text { Self report + } \\
\text { examination }\end{array}$} & \multirow[t]{2}{*}{ Point } & $60-69$ & & & 0.9 & L \\
\hline & & & & & & & & & $70+$ & & & 0.9 & \\
\hline \multirow{3}{*}{$\begin{array}{l}\text { Carmona [11] } \\
2001 \text { Spain }\end{array}$} & \multirow{3}{*}{$\begin{array}{l}\text { ( } 20+\text { yo), a stratified multistage } \\
\text { cluster sampling from the } \\
\text { censuses of } 20 \text { municipalities. } \\
\text { Postal questionnaire + interview } \\
\text { by a rheumatologist }\end{array}$} & \multirow[t]{3}{*}{2192} & \multirow[t]{3}{*}{1014} & \multirow[t]{3}{*}{1178} & \multirow[t]{3}{*}{73} & \multirow{3}{*}{$\begin{array}{l}\text { ACR criteria, } \\
\text { based on modified } \\
\text { questionnaire }\end{array}$} & \multirow[t]{3}{*}{ Self report } & \multirow[t]{3}{*}{ Point } & $60-69$ & & & 1.0 & L \\
\hline & & & & & & & & & 70-79 & & & 0.5 & \\
\hline & & & & & & & & & $80+$ & & & 2.7 & \\
\hline $\begin{array}{l}\text { Collerton [12] } \\
2009 \text { UK }\end{array}$ & $\begin{array}{l}\text { 2006-7, (85+ yo), all people born } \\
\text { in 1921, permanently registered } \\
\text { with a participating GP in } \\
\text { Newcastle upon Tyne or North } \\
\text { Tyneside primary care trusts } \\
\text { (the Newcastle } 85+\text { Study). } \\
\text { Medical records at the GP }\end{array}$ & 853 & 323 & 530 & 59 & Not stated & Medical record & Point & 85 & 0.5 & 5.1 & 3.5 & $U$ \\
\hline \multirow{3}{*}{$\begin{array}{l}\text { Englund [13] } \\
2010 \text { Sweden }\end{array}$} & \multirow{3}{*}{$\begin{array}{l}\text { 2008, }(20+\text { yo), all patients } \\
\text { diagnosed with RA registered } \\
\text { in the Skåne Health Care } \\
\text { Register, southern Sweden. } \\
\text { Data from a national registry }\end{array}$} & \multirow[t]{3}{*}{931316} & \multirow[t]{3}{*}{$27 \%$} & $73 \%$ & N/A & Diagnosis of RA & National register & Point & $65-74$ & 1.0 & 1.9 & 1.5 & L \\
\hline & & & & & & $\begin{array}{l}\text { given by a specialist } \\
\text { in rheumatology or }\end{array}$ & & & $75-84$ & 1.0 & 1.7 & 1.5 & \\
\hline & & & & & & internal medicine & & & $85+$ & 1.2 & 1.2 & 1.1 & \\
\hline Hanova [14] & 2002, (16+ yo), all patients & $?$ & $?$ & $?$ & N/A & ACR 1987 clinical & Medical record & Point & $60-69$ & 0.6 & 2.3 & & $U$ \\
\hline $\begin{array}{l}2000 \text { Czech } \\
\text { Republic }\end{array}$ & $\begin{array}{l}\text { diagnosed betore } 28 t h \text { rebruary } \\
\text { reported by all }\end{array}$ & & & & & & & & $70-79$ & 0.6 & 2.9 & & \\
\hline & $\begin{array}{l}\text { rheumatologists, other specialists, } \\
\text { and almost all GPs. Medical } \\
\text { records from GPs. }\end{array}$ & & & & & & & & $80+$ & 0.5 & 0.8 & & \\
\hline Laiho [15] & $1989,(75,80 \& 85$ yo), a & 1317 & 484 & 833 & $76-96$ & ACR 1987 clinical & Self report + & Point & 75 & 2.8 & 1.2 & 1.7 & $U$ \\
\hline & $\begin{array}{l}\text { computer-generated random } \\
\text { sample from the population }\end{array}$ & & & & & & examination & & 80 & 0 & 1.4 & 1.0 & \\
\hline & $\begin{array}{l}\text { register, Helsinki \& Vantaa } \\
\text { (the Helsinki Ageing Study \& } \\
\text { the Vantaa study). Interview } \\
\text { and examination }\end{array}$ & & & & & & & & 85 & 0 & 1.3 & 1.0 & \\
\hline Neovius [16] & 2008, (16+ yo), patients with a & 58102 & $?$ & $?$ & $?$ & Any visit listing an & National register & Point & $60-69$ & 0.9 & 2.1 & 1.5 & L \\
\hline & $\begin{array}{l}\text { Clinical visit listing an RA } \\
\text { diagnosis were identified in }\end{array}$ & & & & & $\begin{array}{l}\text { RA diagnosis was } \\
\text { used to define RA. }\end{array}$ & & & 70-79 & 1.3 & 2.6 & 2.0 & \\
\hline & $\begin{array}{l}\text { inpatient and outpatient specialist } \\
\text { care in the National Patient } \\
\text { Register (1964-2007) together } \\
\text { with patients listed in the } \\
\text { Swedish Rheumatology } \\
\text { Quality Register (SRQ; } \\
\text { 1995-2007). National register }\end{array}$ & & & & & & & & $80+$ & 1.5 & 2.7 & 2.2 & \\
\hline
\end{tabular}


Table 4 Description of studies on rheumatoid arthritis (RA) (Continued)

\begin{tabular}{|c|c|c|c|c|c|c|c|c|c|c|c|c|c|}
\hline \multirow{3}{*}{$\begin{array}{l}\text { Ollivier [17] } \\
2004 \text { France }\end{array}$} & \multirow{3}{*}{$\begin{array}{l}\text { 1996, (18+ yo), a random } \\
\text { sample from the official list } \\
\text { of phone numbers in Brittany. } \\
\text { Telephone interviews by a } \\
\text { rheumatologist }\end{array}$} & \multirow[t]{3}{*}{1672} & \multirow[t]{3}{*}{0} & \multirow[t]{3}{*}{1672} & \multirow[t]{3}{*}{92} & \multirow{3}{*}{$\begin{array}{l}\text { ACR } 1987 \text { clinical } \\
\text { criteria }\end{array}$} & \multirow{3}{*}{$\begin{array}{l}\text { Self report + } \\
\text { examination }\end{array}$} & \multirow[t]{3}{*}{ Point } & \multicolumn{2}{|l|}{$60-69$} & \multicolumn{2}{|l|}{1.5} & \multirow[t]{3}{*}{ L } \\
\hline & & & & & & & & & $70-79$ & & 1.1 & & \\
\hline & & & & & & & & & 80-89 & & 1.4 & & \\
\hline \multirow{2}{*}{$\begin{array}{l}\text { Picavet [18] } \\
2003 \text { The } \\
\text { Netherlands }\end{array}$} & \multirow[b]{2}{*}{$\begin{array}{l}\text { 1998, ( } 25+\text { yo), a } 6 \text { months } \\
\text { follow-up on a baseline stratified } \\
\text { random sample taken from the } \\
\text { population register (the Dutch } \\
\text { population-based } \\
\text { Musculoskeletal Complaints } \\
\text { and Consequences Cohort } \\
\text { study, DMC3-study). Postal } \\
\text { questionnaire }\end{array}$} & \multirow[t]{2}{*}{2338} & \multirow[t]{2}{*}{$?$} & \multirow[t]{2}{*}{$?$} & \multirow[t]{2}{*}{85} & \multirow{2}{*}{$\begin{array}{l}\text { "Please indicate whether } \\
\text { a physician or medical } \\
\text { specialist has ever told } \\
\text { you that you have one } \\
\text { or more of the following } \\
\text { diseases [RA]" }\end{array}$} & \multirow[t]{2}{*}{ Self repot } & \multirow[t]{2}{*}{ Point } & \multicolumn{3}{|l|}{$65-74$} & 6 & \multirow[t]{2}{*}{$\mathrm{H}$} \\
\hline & & & & & & & & & \multicolumn{2}{|l|}{$75+$} & & 10 & \\
\hline $\begin{array}{l}\text { Rasch [19] } \\
2003 \text { USA }\end{array}$ & $\begin{array}{l}\text { 1988-94, }(60+\text { yo), a multistage, } \\
\text { stratified probability sample } \\
\text { representative of the civilian } \\
\text { non-institutionalized population } \\
\text { residing in the } 50 \text { states of } \\
\text { the USA. Home interviews } \\
\text { and examination at mobile } \\
\text { centers }\end{array}$ & 5302 & $?$ & $?$ & 80 & $\begin{array}{l}\text { ACR } 1987 \text { clinical criteria: } \\
3 \text { out of } 6 \text { criteria met } \\
\text { (" } \mathrm{n} \text { of k") }\end{array}$ & $\begin{array}{l}\text { Self report + } \\
\text { laboratory } \\
\text { results }\end{array}$ & Point & $60+$ & $1.6(0.8-2.4)$ & $2.4(1.4-3.4)$ & & L \\
\hline $\begin{array}{l}\text { Rasch [19] } \\
2003 \text { USA }\end{array}$ & $\begin{array}{l}\text { 1988-94, }(60+\text { yo), a multistage, } \\
\text { stratified probability sample } \\
\text { representative of the civilian } \\
\text { non-institutionalized population } \\
\text { residing in the } 50 \text { states of the } \\
\text { USA. Home interviews and } \\
\text { examination at mobile } \\
\text { centers }\end{array}$ & 5302 & $?$ & $?$ & 80 & $\begin{array}{l}\text { ACR } 1987 \text { clinical criteria: } \\
\text { allowing surrogate } \\
\text { classification variables } \\
\text { when a primary } \\
\text { classification variable is } \\
\text { unavailable ("classification } \\
\text { tree") }\end{array}$ & $\begin{array}{l}\text { Self report + } \\
\text { laboratory } \\
\text { results }\end{array}$ & Point & $60+$ & $1.6(0.8-2.4)$ & $2.6(1.6-3.6)$ & & L \\
\hline \multirow{4}{*}{$\begin{array}{l}\text { Riise [20] } \\
2000 \text { Norway }\end{array}$} & \multirow{4}{*}{$\begin{array}{l}1987 \& 1996,(20+) \text {, all records } \\
\text { of patients registered at the } \\
\text { Department of Rheumatology } \\
\text { at the University Hospital of } \\
\text { Tromsø [only } 1996 \text { prevalence } \\
\text { reported here] }\end{array}$} & \multirow[t]{4}{*}{2282} & \multirow[t]{4}{*}{$?$} & \multirow[t]{4}{*}{$?$} & \multirow[t]{4}{*}{$?$} & \multirow{4}{*}{$\begin{array}{l}\text { ACR } 1987 \text { clinical criteria } \\
\text { (ICD-9 diagnoses } 714.0 \\
\text { and } 714.9 \text { ) in medical } \\
\text { records and subsequently } \\
\text { critical reviews by a senior } \\
\text { consultant }\end{array}$} & \multirow[t]{4}{*}{ Medical record } & \multirow[t]{4}{*}{ Point } & $60-69$ & 0.9 & 1.4 & 1.2 & \multirow[t]{4}{*}{ L } \\
\hline & & & & & & & & & $70-79$ & 0.9 & 1.9 & 1.5 & \\
\hline & & & & & & & & & $80-89$ & 1.3 & 1.3 & 1.5 & \\
\hline & & & & & & & & & $90+$ & 0.2 & 0.6 & 0.4 & \\
\hline \multirow{2}{*}{$\begin{array}{l}\text { Symmons [21] } \\
2002 \text { UK }\end{array}$} & $(16+)$, a two-stage stratified & 5424 & $?$ & $?$ & 77 & A modified version of the & Clinical & Point & $65-74$ & $1.5(0.8-3.0)$ & $3.3(1.9-5.9)$ & & $U$ \\
\hline & $\begin{array}{l}\text { in Norfolk (GPs allowed to } \\
\text { exclude certain patients). } \\
\text { Postal questionnaire and } \\
\text { examination at the GP }\end{array}$ & & & & & $\begin{array}{l}\text { symptomatic RA followed } \\
\text { by a clinical assessment }\end{array}$ & & & $75+$ & $3.1(1.7-5.5)$ & $5.4(3.1-9.3)$ & & \\
\hline
\end{tabular}

*Prevalence estimates without decimals are obtained from figures/graphs in the article and should be interpreted with caution.

I: Interview, Q: Questionnaire; E: Examination, R: Register. L: Low, U: Unclear, H: High

GP: General practitioner; ACR: The American College of Rheumatology (ACR clinical criteria for RA [22]). 
Table 5 Description of studies on osteoarthritis (OA)

\begin{tabular}{|c|c|c|c|c|c|c|c|c|c|c|c|c|c|c|}
\hline & \multirow{2}{*}{$\begin{array}{l}\text { First author } \\
\text { Publ. year } \\
\text { Country }\end{array}$} & \multirow{2}{*}{$\begin{array}{l}\text { Study design / } \\
\text { Population /Method } \\
\text { of collection }\end{array}$} & \multicolumn{3}{|c|}{ Sample size } & \multirow{2}{*}{$\begin{array}{l}\text { Crude } \\
\text { response } \\
\text { rate }(\%)\end{array}$} & \multirow{2}{*}{$\begin{array}{l}\text { Outcome } \\
\text { definition }\end{array}$} & \multirow{2}{*}{$\begin{array}{l}\text { Outcome } \\
\text { assessment } \\
\text { method }\end{array}$} & \multirow{2}{*}{$\begin{array}{l}\text { Prevalence } \\
\text { period }\end{array}$} & \multirow[t]{2}{*}{ Age } & \multicolumn{3}{|c|}{ Prevalence* $(95 \% \mathrm{Cl})$} & \multirow{2}{*}{$\begin{array}{l}\text { Risk } \\
\text { of } \\
\text { bias }\end{array}$} \\
\hline & & & Total & $M$ & $F$ & & & & & & $M$ & $F$ & Total & \\
\hline \multirow[t]{3}{*}{ Knee, symptomatic } & \multirow{3}{*}{$\begin{array}{l}\text { Carmona [11] } \\
2001 \text { Spain }\end{array}$} & \multirow{3}{*}{$\begin{array}{l}\text { ( } 20+\text { yo), a stratified } \\
\text { multistage cluster } \\
\text { sample from the } \\
\text { censuses of } 20 \\
\text { municipalities. } \\
\text { Postal } \\
\text { questionnaire + } \\
\text { interview } \\
\text { (rheumatologist) }\end{array}$} & \multirow[t]{3}{*}{2192} & \multirow[t]{3}{*}{1014} & \multirow[t]{3}{*}{1178} & \multirow[t]{3}{*}{73} & \multirow{3}{*}{$\begin{array}{l}\text { ACR clinical } \\
\text { criteria }\end{array}$} & \multirow[t]{3}{*}{ Self report } & \multirow[t]{3}{*}{ Point } & $60-69$ & & & 28.1 & L \\
\hline & & & & & & & & & & 70-79 & & & 33.7 & \\
\hline & & & & & & & & & & $80+$ & & & 21.3 & \\
\hline \multirow[t]{3}{*}{ Knee, symptomatic } & \multirow{3}{*}{$\begin{array}{l}\text { Fernandez-Lopez } \\
\text { [25] } 2008 \text { Spain }\end{array}$} & \multirow{3}{*}{$\begin{array}{l}\text { 2000, ( } 20+\text { yo), stratified } \\
\text { poly-stage cluster } \\
\text { sampling from } 20 \\
\text { city censuses, } \\
\text { home visit } \\
\text { questionnaire + } \\
\text { interview } \\
\text { (rheumatologist) }\end{array}$} & \multirow[t]{3}{*}{2192} & \multirow[t]{3}{*}{1014} & \multirow[t]{3}{*}{1178} & \multirow[t]{3}{*}{73} & \multirow{3}{*}{$\begin{array}{l}\text { ACR clinical } \\
\text { criteria }\end{array}$} & \multirow[t]{3}{*}{ Self report } & \multirow[t]{3}{*}{ Point } & $60-69$ & 18.1 & 37.2 & 28.1 & \multirow[t]{3}{*}{$L$} \\
\hline & & & & & & & & & & $70-79$ & 16.7 & 44.1 & 33.7 & \\
\hline & & & & & & & & & & $80+$ & 14.3 & 25.5 & 21.3 & \\
\hline Knee, symptomatic & $\begin{array}{l}\text { Mannoni [30] } \\
2003 \text { Italy }\end{array}$ & $\begin{array}{l}\text { 1995, (65+ yo), the } \\
\text { entire population } \\
\text { of } 65+\text { yo in } \\
\text { Dicomano (The } \\
\text { ICARe Dicomano } \\
\text { study). Home interview } \\
\text { and examination } \\
\text { (geriatrician) }\end{array}$ & 697 & 406 & 291 & 81 & $\begin{array}{l}\text { ACR clinical } \\
\text { criteria }\end{array}$ & $\begin{array}{l}\text { Clinical } \\
\text { examination }\end{array}$ & Point & $65+$ & & & 29.8 & L \\
\hline \multirow[t]{2}{*}{ Knee, symptomatic } & \multirow{2}{*}{$\begin{array}{l}\text { Picavet [18] } \\
2003 \text { The } \\
\text { Netherlands }\end{array}$} & \multirow[b]{2}{*}{$\begin{array}{l}\text { 1998, ( } 25+\text { yo), a } 6 \\
\text { months follow-up on } \\
\text { a baseline stratified } \\
\text { random sample taken } \\
\text { from the population } \\
\text { register (the DMC3- } \\
\text { study). Postal } \\
\text { questionnaire }\end{array}$} & \multirow[t]{2}{*}{2338} & $?$ & $?$ & 85 & "Please indicate & Self report & Point & $65-74$ & & & 27 & $\mathrm{H}$ \\
\hline & & & & & & & $\begin{array}{l}\text { whether a physician } \\
\text { or medical specialist } \\
\text { has ever told you } \\
\text { that you have one } \\
\text { or more of the } \\
\text { following diseases } \\
\text { [OA]" }\end{array}$ & & & $75+$ & & & 28 & \\
\hline Knee, radiographic & Jordan [27] & 1991-7, (45+ yo), & 3690 & $?$ & $?$ & 72 & $K-L \geq 2$ & Radiograph & Point & $65-74$ & & & $36.1(33.8-38.6)$ & U \\
\hline & 2007 USA & $\begin{array}{l}\text { stratified simple random } \\
\text { sampling of streets as } \\
\text { primary sampling units } \\
\text { and stratified subsampling } \\
\text { of Caucasian women } \\
\text { age } 65 \text { years or older } \\
\text { residents of one of } 6 \\
\text { townships (the Johnston } \\
\text { County Osteoarthritis } \\
\text { Project). Home interview } \\
\text { and clinical examination }\end{array}$ & & & & & & & & $75+$ & & & $49.9(45.4-54.4)$ & \\
\hline
\end{tabular}




\begin{tabular}{|c|c|c|c|c|c|c|c|c|c|c|c|c|c|c|}
\hline \multirow[t]{3}{*}{ Knee, radiographic } & \multirow{3}{*}{$\begin{array}{l}\text { Kim [28] } 2010 \\
\text { South Korea }\end{array}$} & \multirow{3}{*}{$\begin{array}{l}2007,(50+\text { yo), a } \\
\text { follow-up study of a } \\
\text { random proportional } \\
\text { sample from the Korean } \\
\text { National Census of elderly } \\
\text { community residents in } \\
\text { Chuncheon city. Home } \\
\text { interview, Questionnaire } \\
\text { and examination }\end{array}$} & \multirow[t]{3}{*}{504} & \multirow[t]{3}{*}{230} & \multirow[t]{3}{*}{274} & \multirow[t]{3}{*}{55} & \multirow[t]{3}{*}{$K-L \geq 2$} & \multirow[t]{3}{*}{ Radiograph } & \multirow[t]{3}{*}{ Point } & 60-69 & 4 & 40 & 26 & U \\
\hline & & & & & & & & & & 70-79 & 18 & 65 & 42 & \\
\hline & & & & & & & & & & 80-89 & 34 & 98 & 65 & \\
\hline \multirow[t]{4}{*}{ Knee, radiographic } & \multirow{4}{*}{$\begin{array}{l}\text { Muraki [31] } \\
2009 \text { Japan }\end{array}$} & \multirow{4}{*}{$\begin{array}{l}\text { 2002, (65+yo), random } \\
\text { samples of community- } \\
\text { dwelling people from } \\
\text { listings of resident } \\
\text { registration in three } \\
\text { communities (Itabashi-ku, } \\
\text { Hidakagawa-cho, } \\
\text { Taiji-cho). Interview, } \\
\text { Questionnaire and } \\
\text { examination }\end{array}$} & \multirow[t]{4}{*}{2282} & \multirow[t]{4}{*}{817} & \multirow[t]{4}{*}{1465} & \multirow[t]{4}{*}{$29-76$} & \multirow[t]{4}{*}{$K-L \geq 2$} & \multirow[t]{4}{*}{ Radiograph } & \multirow[t]{4}{*}{ Point } & 65-69 & 42 & 61 & & \multirow[t]{4}{*}{ U } \\
\hline & & & & & & & & & & 70-74 & 46 & 71 & & \\
\hline & & & & & & & & & & 75-79 & 51 & 74 & & \\
\hline & & & & & & & & & & $80+$ & 53 & 81 & & \\
\hline \multirow[t]{3}{*}{ Knee, radiographic } & \multirow{3}{*}{$\begin{array}{l}\text { Sudo [32] } \\
2008 \text { Japan }\end{array}$} & \multirow{3}{*}{$\begin{array}{l}(65+\text { yo), all community } \\
\text { inhabitants recruited in } \\
\text { Miyagawa village, in } \\
\text { central Mie Prefecture. } \\
\text { Questionnaire and } \\
\text { interview (hospital) }\end{array}$} & \multirow[t]{3}{*}{598} & \multirow[t]{3}{*}{205} & \multirow[t]{3}{*}{393} & \multirow[t]{3}{*}{40} & \multirow[t]{3}{*}{$K-L \geq 2$} & \multirow[t]{3}{*}{ Radiograph } & \multirow[t]{3}{*}{ Point } & $65-74$ & 14 & 33 & & u \\
\hline & & & & & & & & & & 75-84 & 26 & 41 & & \\
\hline & & & & & & & & & & $85+$ & 23 & 47 & & \\
\hline Knee, radiographic & Yoshida [34] & 2000, (40+ yo), all & 586 & & 586 & 30 & $K-L \geq 2$ & Radiograph & Point & 63-69 & & 35.8 & & U \\
\hline & & $\begin{array}{l}\text { women Identified by } \\
\text { the municipal electroral }\end{array}$ & & & & & & & & 70-79 & & 54.0 & & \\
\hline & & $\begin{array}{l}\text { list of Oshima town, } \\
\text { Nagasaki (The Hizen- } \\
\text { Oshima Study). } \\
\text { examination }\end{array}$ & & & & & & & & 80-89 & & 63.3 & & \\
\hline Knee, radiographic & Yoshimura [33] & $2005-7,(40+$ yo), recruited & 3040 & 1061 & 1979 & 76 & $K-L \geq 2$ & Radiograph & Point & 60-69 & 35.2 & 57.1 & & u \\
\hline & & $\begin{array}{l}\text { from the resident- } \\
\text { registration lists of the }\end{array}$ & & & & & & & & 70-79 & 48.2 & 71.9 & & \\
\hline & & $\begin{array}{l}\text { Hidakagawa \& } \\
\text { Taiji regions or from a } \\
\text { randomly selected } \\
\text { cohort study from the } \\
\text { Itabashi (Tokyo) Ward } \\
\text { resident registration } \\
\text { database (the ROAD } \\
\text { study). Examination }\end{array}$ & & & & & & & & $80+$ & 51.6 & 80.7 & & \\
\hline Knee, symptomatic + & Andrianakos [23] & 1966-99, (19+ yo), the & 8740 & 4269 & 4471 & 82 & ACR clinical & Self report + & Point & 60-64 & 5.4 & 21.4 & 13.3 & L \\
\hline & & $\begin{array}{l}\text { total adult population } \\
\text { in } 7 \text { mixed }\end{array}$ & & & & & $\begin{array}{l}\text { Criteria + radiograph } \\
\text { (unknown }\end{array}$ & & & $65-69$ & 8.4 & 21.1 & 15.3 & \\
\hline & & communities + random & & & & & definition) & & & 70-74 & 11.7 & 28.0 & 20.4 & \\
\hline & & 2 mixed communities & & & & & & & & 75-79 & 19.3 & 33.3 & 27.6 & \\
\hline
\end{tabular}




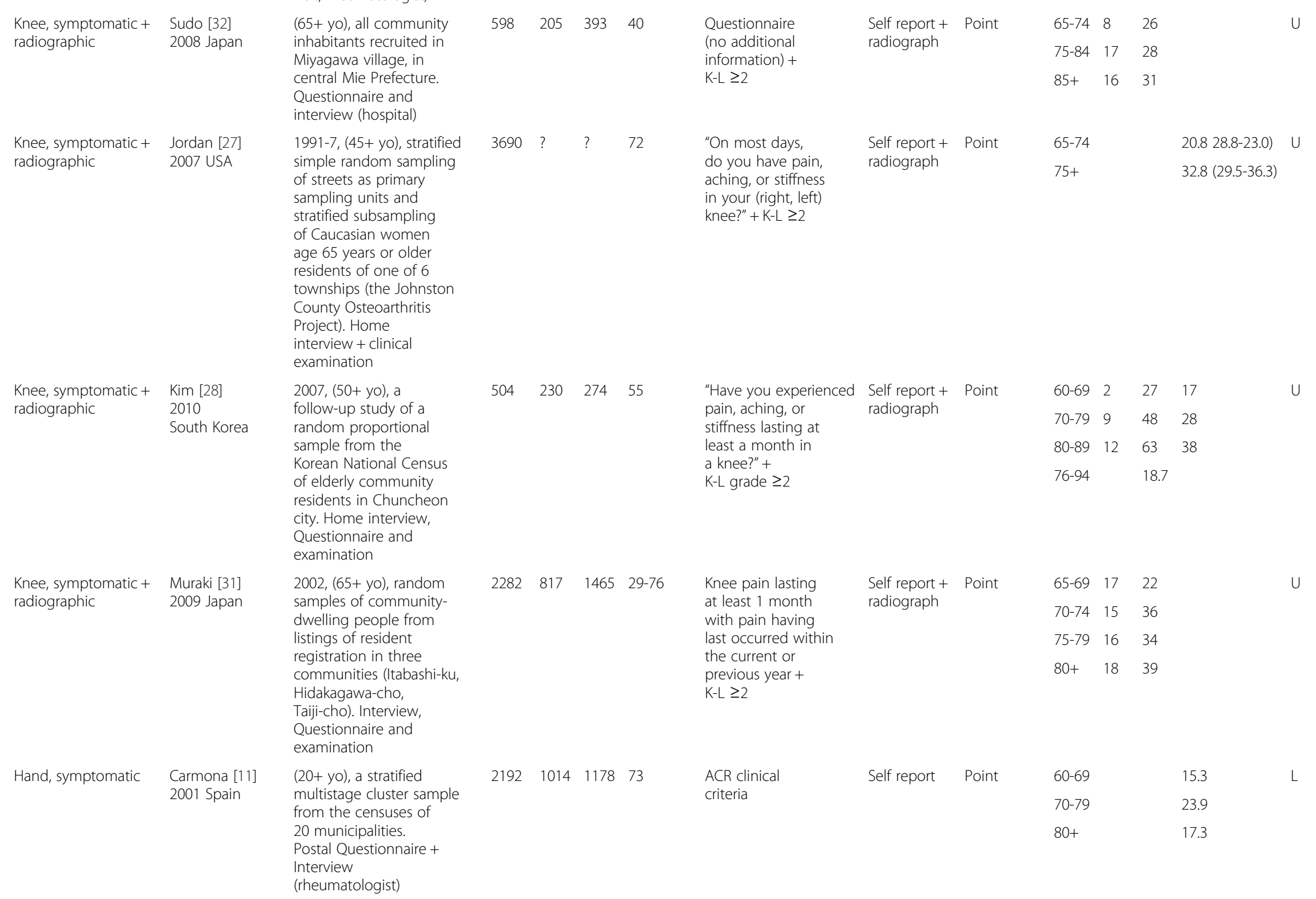

\section{(the ESORDIG study).}

Interview,

Questionnaire and

examination (home

visit, rheumatologist)

7, (45+ yo), stratified

m sampling

llow-up study of a

Korean National Census

city. Home interview,

Qxamination

egistration in three

Hidakagawa-cho,
ACR clinic

Sefreport

$80+$ 
Table 5 Description of studies on osteoarthritis (OA) (Continued)

\begin{tabular}{|c|c|c|c|c|c|c|c|c|c|c|c|c|c|c|}
\hline Hand, symptomatic & $\begin{array}{l}\text { Mannoni [37] } \\
2003 \text { Italy }\end{array}$ & $\begin{array}{l}\text { 1995, (65+ yo), the } \\
\text { entire population of } \\
65+\text { yo in Dicomano } \\
\text { (The ICARe Dicomano } \\
\text { study). Home interview } \\
\text { and examination } \\
\text { (geriatician) }\end{array}$ & 697 & 406 & 291 & 81 & $\begin{array}{l}\text { ACR clinical; } \\
\text { criteria }\end{array}$ & $\begin{array}{l}\text { Clinical } \\
\text { examination }\end{array}$ & Point & $65+$ & & & 14.9 & $L$ \\
\hline \multirow[t]{3}{*}{ Hand, radiographic } & \multirow{3}{*}{$\begin{array}{l}\text { Dillon [24] } \\
2007 \text { USA }\end{array}$} & \multirow{3}{*}{$\begin{array}{l}\text { 1991-4, }(60+\text { yo), a } \\
\text { multistage, cluster and } \\
\text { stratified representative } \\
\text { sample of US civilians } \\
\text { (NHANES III). Home } \\
\text { Questionnaire and } \\
\text { Interview, Examination } \\
\text { in mobile examination } \\
\text { centre }\end{array}$} & \multirow[t]{3}{*}{2498} & \multirow[t]{3}{*}{$?$} & \multirow[t]{3}{*}{$?$} & \multirow[t]{3}{*}{62} & \multirow{3}{*}{$\begin{array}{l}\text { NHANES III criteria, } \\
\text { but with no history } \\
\text { of persistent } \\
\text { symptoms }\end{array}$} & \multirow{3}{*}{$\begin{array}{l}\text { Self report + } \\
\text { clinical } \\
\text { examination }\end{array}$} & \multirow[t]{3}{*}{ Point } & $60-69$ & & & 31.5 & \multirow[t]{3}{*}{ L } \\
\hline & & & & & & & & & & 70-79 & & & 43.9 & \\
\hline & & & & & & & & & & $80+$ & & & 41.2 & \\
\hline \multirow[t]{5}{*}{ Hand, radiographic } & \multirow{5}{*}{$\begin{array}{l}\text { Haugen [26] } \\
2011 \text { Norway }\end{array}$} & \multirow{5}{*}{$\begin{array}{l}\text { 1992-5 \& 2002-5, } \\
\text { (28-92 yo), baseline } \\
\text { data from the 1992-5 } \\
\text { Community cohort of } \\
\text { the Framingham } \\
\text { Heart Study selected } \\
\text { through random-digit } \\
\text { dialing and from the } \\
\text { 2002-5 Offspring } \\
\text { cohort, Massachusetts. } \\
\text { Postal questionnaire + } \\
\text { examination }\end{array}$} & \multirow[t]{5}{*}{2300} & \multirow[t]{5}{*}{$?$} & \multirow[t]{5}{*}{$?$} & \multirow[t]{5}{*}{43} & \multirow{5}{*}{$\begin{array}{l}\text { Modified K-L grade } \\
\geq 2 \text { ( } 2=\text { mild HOA, } \\
\text { i.e. small OP(s) } \\
\text { and/or mild } \\
\text { JSN, sclerosis } \\
\text { may be present) }\end{array}$} & \multirow[t]{5}{*}{ Radiograph } & \multirow[t]{5}{*}{ Point } & $60-64$ & 56 & 63 & & \multirow[t]{5}{*}{ U } \\
\hline & & & & & & & & & & $65-69$ & 71 & 82 & & \\
\hline & & & & & & & & & & $70-74$ & 78 & 91 & & \\
\hline & & & & & & & & & & $75-79$ & 72 & 92 & & \\
\hline & & & & & & & & & & $80+$ & 96 & 100 & & \\
\hline \multirow[t]{3}{*}{ Hand, radiographic } & \multirow{3}{*}{$\begin{array}{l}\text { Kwok [29] } \\
2011 \text { The } \\
\text { Netherlands }\end{array}$} & \multirow{3}{*}{$\begin{array}{l}\text { 1997-3, (55+ yo), } \\
\text { responders from } \\
\text { follow-up of 1990-3 } \\
\text { random sample of } \\
\text { inhabitants living } \\
\text { in the Ommoord } \\
\text { district, Rotterdam } \\
\text { (the Rotterdam } \\
\text { Study). Questionnaire } \\
\text { and Interview } \\
\text { (home), Examination }\end{array}$} & \multirow[t]{3}{*}{3430} & \multirow[t]{3}{*}{1509} & \multirow[t]{3}{*}{1921} & \multirow[t]{3}{*}{43} & \multirow{3}{*}{$\begin{array}{l}\text { 'Mild' OA defined } \\
\text { as } K L \text { grade } \geq 2 \\
\text { in at least one } \\
\text { finger joint }\end{array}$} & \multirow[t]{3}{*}{ Radiograph } & \multirow[t]{3}{*}{ Point } & $65-74$ & 56.3 & 68.4 & 62.9 & \multirow[t]{3}{*}{ U } \\
\hline & & & & & & & & & & $75-84$ & 63.3 & 78.9 & 72.8 & \\
\hline & & & & & & & & & & $85+$ & 66.7 & 68.4 & 67.9 & \\
\hline Hand, symptomatic + & Andrianakos [23] & 1966-99, (19+ yo), the & 8740 & 4269 & 4471 & 82 & ACR clinical & Self report + & Point & $60-64$ & 0.9 & 7.0 & 3.9 & L \\
\hline & 2006 Greece & $\begin{array}{l}\text { total adult population } \\
\text { in } 7 \text { mixed }\end{array}$ & & & & & $\begin{array}{l}\text { Criteria + radiograph } \\
\text { (unknown }\end{array}$ & radiograph & & $65-69$ & 2.1 & 8.8 & 5.7 & \\
\hline & & communities + & & & & & definition) & & & $70-74$ & 3.3 & 7.8 & 5.8 & \\
\hline & & another 2 mixed & & & & & & & & 75-79 & 4.0 & 8.1 & 6.5 & \\
\hline & & $\begin{array}{l}\text { communities (the } \\
\text { ESORDIG study). } \\
\text { Interview, Questionnaire } \\
\text { and examination } \\
\text { (home visit, } \\
\text { rheumatologist). }\end{array}$ & & & & & & & & $80+$ & 1.8 & 5.5 & 4.2 & \\
\hline
\end{tabular}




\begin{tabular}{|c|c|c|c|c|c|c|c|c|c|c|c|c|c|c|}
\hline \multirow{3}{*}{$\begin{array}{l}\text { Hand, symptomatic + } \\
\text { radiographic }\end{array}$} & \multirow{3}{*}{$\begin{array}{l}\text { Dillon [24] } \\
2007 \text { USA }\end{array}$} & \multirow{3}{*}{$\begin{array}{l}\text { 1991-4, (60+ yo), a } \\
\text { multistage, cluster and } \\
\text { stratified representative } \\
\text { sample of US civilians } \\
\text { (NHANES III). Home } \\
\text { Questionnaire and } \\
\text { Interview, Examination } \\
\text { (mobile examination } \\
\text { centre). }\end{array}$} & \multirow[t]{3}{*}{2498} & \multirow[t]{3}{*}{$?$} & \multirow[t]{3}{*}{$?$} & \multirow[t]{3}{*}{62} & \multirow{3}{*}{$\begin{array}{l}\text { NHANES III } \\
\text { criteria }\end{array}$} & \multirow{3}{*}{$\begin{array}{l}\text { Self report + } \\
\text { clinical } \\
\text { examination }\end{array}$} & \multirow[t]{3}{*}{ Point } & \multicolumn{3}{|l|}{$60-69$} & 6.1 & \multirow[t]{3}{*}{$\mathrm{L}$} \\
\hline & & & & & & & & & & $70-79$ & & & 9.9 & \\
\hline & & & & & & & & & & $80+$ & & & 9.7 & \\
\hline \multirow{3}{*}{$\begin{array}{l}\text { Hand, symptomatic + } \\
\text { radiographic }\end{array}$} & \multirow{3}{*}{$\begin{array}{l}\text { Kwok [29] } \\
2011 \text { The } \\
\text { Netherlands }\end{array}$} & \multirow{3}{*}{$\begin{array}{l}\text { 1997-3, (55+ yo), responders } \\
\text { from follow-up of } \\
\text { 1990-3 random sample } \\
\text { of inhabitants living in } \\
\text { the Ommoord district, } \\
\text { Rotterdam (the } \\
\text { Rotterdam Study). } \\
\text { Questionnaire and } \\
\text { Interview (home), } \\
\text { Examination }\end{array}$} & \multirow{3}{*}{3430} & \multirow[t]{3}{*}{1509} & \multirow[t]{3}{*}{1921} & \multirow[t]{3}{*}{43} & \multirow{3}{*}{$\begin{array}{l}\text { 'Did you have } \\
\text { any pain in the } \\
\text { right or left hand } \\
\text { during the last } \\
\text { month?' + 'Mild' } \\
\mathrm{OA} \text { defined as } \\
\mathrm{KL} \text { grade } \geq 2 \text { in } \\
\text { at least one } \\
\text { finger joint }\end{array}$} & \multirow{3}{*}{$\begin{array}{l}\text { Self report + } \\
\text { radiograph }\end{array}$} & \multirow[t]{3}{*}{ Point } & $65-74$ & 6.1 & 18.9 & 13.1 & \multirow[t]{3}{*}{ U } \\
\hline & & & & & & & & & & $75-84$ & 5.3 & 14.2 & 10.7 & \\
\hline & & & & & & & & & & $85+$ & 0.0 & 21.1 & 14.3 & \\
\hline \multirow{3}{*}{$\begin{array}{l}\text { Hand, symptomatic + } \\
\text { radiographic }\end{array}$} & \multirow{3}{*}{$\begin{array}{l}\text { Zhang [36] } \\
2002 \text { USA }\end{array}$} & \multirow{3}{*}{$\begin{array}{l}\text { 1992-3, (71+ yo), all } \\
\text { participants from } \\
\text { the original cohort in } \\
1948 \text { aged } 26-62 \text { (the } \\
\text { Framingham Study). } \\
\text { Questionnaire and } \\
\text { Interview, } \\
\text { Examination }\end{array}$} & \multirow[t]{3}{*}{1032} & \multirow[t]{3}{*}{369} & \multirow[t]{3}{*}{663} & \multirow[t]{3}{*}{89} & \multirow{3}{*}{$\begin{array}{l}\text { "On most days, } \\
\text { do you have pain, } \\
\text { aching, or stiffness } \\
\text { in any of your } \\
\text { joints?" }+ \text { K-L } \geq 2\end{array}$} & \multirow{3}{*}{$\begin{array}{l}\text { Self report + } \\
\text { radiograph }\end{array}$} & \multirow[t]{3}{*}{ Point } & $71-74$ & 16.4 & 27.2 & & U \\
\hline & & & & & & & & & & $75-79$ & 11.9 & 26.1 & & \\
\hline & & & & & & & & & & $80+$ & 13.5 & 26.0 & & \\
\hline Hip, symptomatic & Picavet [18] & 1998, (25+ yo), a 6 & 2338 & $?$ & $?$ & 85 & "Please indicate & Self report & Life time & $65-74$ & & & 17 & $\mathrm{H}$ \\
\hline & Netherlands & $\begin{array}{l}\text { montss follow-up on } \\
\text { random sample taken } \\
\text { from the population } \\
\text { register (the DMC3- } \\
\text { study). Postal } \\
\text { questionnaire }\end{array}$ & & & & & $\begin{array}{l}\text { or medical specialist } \\
\text { has ever told you } \\
\text { that you have one } \\
\text { or more of the } \\
\text { following diseases } \\
{[\mathrm{OA}]^{\prime}}\end{array}$ & & & $75+$ & & & 22 & \\
\hline Hip, symptomatic & $\begin{array}{l}\text { Mannoni [37] } \\
2003 \text { Italy }\end{array}$ & $\begin{array}{l}\text { 1995, (65+ yo), the } \\
\text { entire population of } \\
65+\text { yo in Dicomano } \\
\text { (The ICARe Dicomano } \\
\text { study). Interview and } \\
\text { examination (home } \\
\text { visit, geriatrician) }\end{array}$ & 697 & 406 & 291 & 81 & $\begin{array}{l}\text { ACR clinical } \\
\text { criteria }\end{array}$ & $\begin{array}{l}\text { Clinical } \\
\text { examination }\end{array}$ & Point & $65+$ & & & 7.7 & L \\
\hline Hip, symptomatic + & Andrianakos [23] & 1966-99, $(19+$ yo), the & 8740 & 4269 & 4471 & 82 & ACR clinical & Self report + & Point & $60-64$ & 0.7 & 3.5 & 2.1 & L \\
\hline & zovo Gleece & in 7 mixed & & & & & (unknown & Taurograpil & & $65-69$ & 0.5 & 4.1 & 2.4 & \\
\hline & & communities + random & & & & & definition) & & & 70-74 & 1.2 & 3.9 & 2.6 & \\
\hline & & 2 mixed communities & & & & & & & & 75-79 & 0.6 & 4.3 & 3.0 & \\
\hline & & (the ESORDIG & & & & & & & & $80+$ & 0.6 & 2.8 & 1.8 & \\
\hline
\end{tabular}


Table 5 Description of studies on osteoarthritis (OA) (Continued)

\begin{tabular}{|c|c|c|c|c|c|c|c|c|c|c|c|c|c|}
\hline & & $\begin{array}{l}\text { study). Interview, } \\
\text { Questionnaire and } \\
\text { examination (home } \\
\text { visit, geriatrician) }\end{array}$ & & & & & & & & & & & \\
\hline \multirow{3}{*}{$\begin{array}{l}\text { Lumbar spine } \\
\text { radiographic }\end{array}$} & \multirow{3}{*}{$\begin{array}{l}\text { Yoshimura [33] } \\
2009 \text { Japan }\end{array}$} & \multirow{3}{*}{$\begin{array}{l}2005-7,(40+\text { yo), recruited } \\
\text { from the resident- } \\
\text { registration lists of the } \\
\text { Hidakagawa \& Taiji } \\
\text { regions or from a } \\
\text { randomly selected } \\
\text { cohort study from the } \\
\text { Itabashi (Tokyo) Ward } \\
\text { resident registration } \\
\text { database (the ROAD } \\
\text { study). Examination }\end{array}$} & \multirow[t]{3}{*}{3040} & \multirow[t]{3}{*}{1061} & \multirow[t]{3}{*}{1979} & \multirow[t]{3}{*}{76} & \multirow[t]{3}{*}{$K-L \geq 3$} & \multirow[t]{3}{*}{ Radiograph } & \multirow[t]{3}{*}{ Point } & $60-69$ & 74.6 & 64.3 & U \\
\hline & & & & & & & & & & 70-79 & 85.3 & 76.1 & \\
\hline & & & & & & & & & & $80+$ & 89.9 & 79.6 & \\
\hline \multirow{2}{*}{$\begin{array}{l}\text { Lumbar spine } \\
\text { radiographic }\end{array}$} & \multirow{2}{*}{$\begin{array}{l}\text { Yoshimura [35] } \\
2009 \text { Japan }\end{array}$} & \multirow{2}{*}{$\begin{array}{l}\text { 1990, (40-79yo), all } \\
\text { inhabitants from the } \\
\text { register of residents in } \\
\text { Miyama village were } \\
\text { invited (the Miyama } \\
\text { Study). Examination }\end{array}$} & \multirow[t]{2}{*}{400} & \multirow[t]{2}{*}{200} & \multirow[t]{2}{*}{200} & \multirow[t]{2}{*}{100} & \multirow[t]{2}{*}{$K-L \geq 3$} & \multirow[t]{2}{*}{ Radiograph } & \multirow[t]{2}{*}{ Point } & $60-69$ & 39.6 & 38.0 & \multirow[t]{2}{*}{ U } \\
\hline & & & & & & & & & & 70-79 & 38.3 & 34.7 & \\
\hline
\end{tabular}

*Prevalence estimates without decimals are obtained from figures/graphs in the article and should be interpreted with caution. R: Register. L: Low, U: Unclear, H: High.

GP: General practitioner; ACR: The American College of Rheumatology (ACR clinical criteria for RA [22]). 


\section{Hand $O A$}

Seven studies included data on hand OA [11,23,24,26,29,36,37] with a total of eight prevalence estimates on symptomatic $[11,37]$, radiographic [26,29], and combined symptomatic/ radiographic hand OA [23,24,29,36] (Table 5).

Regardless of hand OA definitions, women had more OA than men and overall, OA increased with age, although several studies also reported a slight decrease in the oldest age groups.

Five studies reported either symptomatic hand OA only [11,37] or radiographic hand OA only [24,26,29], all with different definitions and age ranges. Nevertheless, similar point prevalences were noted: Approximately $15 \%$ of the "younger" elderly population reported symptomatic hand OA. Radiographic hand OA ranged from approximately $56 \%$ in the "youngest" elderly men to $100 \%$ in the oldest women.

The point prevalence estimates of combined symptomatic/radiographic hand OA ranged from approximately $4 \%$ in the "youngest" elderly population to approximately $14 \%$ in the oldest people and were therefore less common than radiographic hand $\mathrm{OA}$ alone.

\section{Prevalence of OP}

Twenty-one studies reported prevalence estimates on OP of which 14 studies measured the bone mineral density (BMD) in five well-defined anatomical areas (lumbar spine/hip, lumbar spine only, hip/femoral neck only, hand, and heel) [33,35,41-52]. Seven studies used other definitions and were mostly based on self reported data [12,18,53-57] (Table 6). Four studies (19\%) were of high risk of bias $[18,47,51,54]$, whereas only two studies (10\%) were of low risk of bias [41,52] (Table 6 and Additional file 4).

Regardless of the anatomical site, a steady increase in $\mathrm{OP}$ with increasing age for all types of OP definitions was seen. Generally, OP was two-three times more common in women than in men.

\section{Lumbar spine $O P$}

Eight studies included data on lumbar OP [33,35,44,45,49-52], all using the WHO BMD T-score of -2.5 SD or less [58], except for two studies [33,51] (Table 6). While the Spanish and Danish OP age related prevalences in women were similar (ranging 17\%-66\%), greater age related variations were noted in women in the Asian countries. For example, in South Korean women, markedly higher estimates across ages (51\%-61\%) were reported by Cui et al. [44] compared to Shin et al. (29\%-48\%) [50].

\section{Hip or femoral neck OP}

Seven studies reported either hip or femoral neck OP $[33,44-46,49,51]$. Fairly similar results were noted in
South Korea and Australia (range: 11\%-37\% for 6079 year olds) $[44,45]$, but the UK and Spanish estimates were slightly lower (range: $7 \%-15 \%$ for $60-74$ year olds) $[46,49]$.

\section{Combined lumbar spine and/or hip OP}

Lumbar spine and/or hip OP was reported in five studies $[41,43,48,49,52]$ which all, except for one study [43], used the WHO bone mineral density (BMD) threshold (T-score) of $-2.5 \mathrm{SD}$ or less (Table 6). The prevalence of OP was slightly higher in Danish women [52] (range: 30\%-92\%) than in Spanish women [49] (range: $23 \%-49 \%)$.

\section{Prevalence of BP}

In all, BP 31 studies were included [11,41,59-87] of which seven (23\%) studies were of low risk of bias $[11,41,73-75,78,80]$ and three $(10 \%)$ of high risk of bias $[59,81,83]$ (Table 7 and Additional file 4).

\section{Low back pain}

Low back pain was reported in 20 studies all with different LBP definitions and with eight different prevalence periods (Table 7) [11,41,59,60,64,66,69,71,74,75,77-83,85-87].

The one-month prevalence was the most common prevalence period reported and ranged between 27\% and $49 \%$. The lowest estimates were based on more restricted definitions, whereas the larger estimates (47-49\%) had less restricted LBP definitions.

Overall, the prevalence estimates increased up to 80 years of age and then dropped slightly after that. With one exception [83], women reported LBP more often than men.

\section{Back pain}

Back pain was used in six studies $[62,63,68,70,72,73]$ on five different prevalence estimates, all with different BP definitions and with a wide range in prevalence estimates. Thus, one-month BP prevalence ranged between $18 \%$ and $29 \%$, and the point prevalence ranged from $27 \%$ to $58 \%$. Interestingly, in two studies where 100 year olds were included, the point and one-month BP was roughly the same (27\%-29\%) [63,70]. Prevalence estimates were all higher among women, but age-related changes are inconclusive as most studies did not demonstrate any major changes across ages.

\section{Neck pain}

Sixteen studies on NP reported six different prevalence periods $[41,61,67-70,73,75-78,82,84-87]$ of which the one-month prevalence was the most commonly used period. No identical NP definitions were used and/or different age intervals were reported, although some definitions and intervals were fairly similar. 
Table 6 Description of studies on osteoporosis (OP)

\begin{tabular}{|c|c|c|c|c|c|c|c|c|c|c|c|c|c|c|}
\hline & \multirow{2}{*}{$\begin{array}{l}\text { First author } \\
\text { Publ. year } \\
\text { Country }\end{array}$} & \multirow{2}{*}{$\begin{array}{l}\text { Study design / } \\
\text { Population /Method } \\
\text { of collection }\end{array}$} & \multicolumn{3}{|c|}{ Sample size } & \multirow{2}{*}{$\begin{array}{l}\text { Crude } \\
\text { response } \\
\text { rate (\%) }\end{array}$} & \multirow{2}{*}{$\begin{array}{l}\text { Outcome } \\
\text { definition }\end{array}$} & \multirow{2}{*}{$\begin{array}{l}\text { Outcome } \\
\text { assessment } \\
\text { method }\end{array}$} & \multirow{2}{*}{$\begin{array}{l}\text { Prevalence } \\
\text { period }\end{array}$} & \multirow[t]{2}{*}{ Age } & \multicolumn{3}{|c|}{ Prevalence* $(95 \% \mathrm{Cl})$} & \multirow{2}{*}{$\begin{array}{l}\text { Risk } \\
\text { of } \\
\text { bias }\end{array}$} \\
\hline & & & Total & $M$ & $F$ & & & & & & $M$ & $F$ & Total & \\
\hline \multirow{2}{*}{$\begin{array}{l}\text { Lumbar } \\
\text { spine } \\
\text { or hip }\end{array}$} & \multirow{2}{*}{$\begin{array}{l}\text { Andrianakos } \\
\text { [41] } 2006 \\
\text { Greece }\end{array}$} & \multirow[b]{2}{*}{$\begin{array}{l}\text { 1966-99, }(19+\text { yo), } \\
\text { the total population } \\
\text { in } 7 \text { mixed } \\
\text { communities + } \\
\text { random sample in } \\
\text { another } 2 \text { mixed } \\
\text { communities. } \\
\text { Examination } \\
\text { (rheumatological } \\
\text { centers) }\end{array}$} & \multirow[t]{2}{*}{8740} & \multirow[t]{2}{*}{4269} & \multirow[t]{2}{*}{4471} & \multirow[t]{2}{*}{82} & \multirow{2}{*}{$\begin{array}{l}\text { WHO BMD } \\
\text { T-score }-2.5 \\
\text { SD or less }\end{array}$} & \multirow[t]{2}{*}{ DXA } & \multirow[t]{2}{*}{ Point } & $59-64$ & & & 7 & L \\
\hline & & & & & & & & & & $69+$ & & & 10 & \\
\hline \multirow{5}{*}{$\begin{array}{l}\text { Lumbar } \\
\text { spine } \\
\text { or hip }\end{array}$} & \multirow{5}{*}{$\begin{array}{l}\text { Bleicher [43] } \\
2010 \text { Australia }\end{array}$} & \multirow{5}{*}{$\begin{array}{l}\text { 2005-07, }(70+\text { yo), } \\
\text { community-dwelling } \\
\text { in three local } \\
\text { government areas } \\
\text { around Sydney } \\
\text { (CHAMP). } \\
\text { Questionnaire + } \\
\text { Examination }\end{array}$} & \multirow[t]{5}{*}{1626} & \multirow[t]{5}{*}{1626} & \multirow[t]{5}{*}{0} & \multirow[t]{5}{*}{45} & \multirow{5}{*}{$\begin{array}{l}\text { Pharmaceutical } \\
\text { Benefits Scheme } \\
\text { criteria for OP: } \\
\text { BMD T-score -3 } \\
\text { SD or less }\end{array}$} & \multirow[t]{5}{*}{ Hologic DXA } & \multirow[t]{5}{*}{ Point } & 70-74 & & & 5.0 & $U$ \\
\hline & & & & & & & & & & 75-79 & & & 4.0 & \\
\hline & & & & & & & & & & $80-84$ & & & 5.0 & \\
\hline & & & & & & & & & & $85-89$ & & & 5.0 & \\
\hline & & & & & & & & & & $90+$ & & & 14.0 & \\
\hline $\begin{array}{l}\text { Lumbar } \\
\text { spine } \\
\text { or hip }\end{array}$ & $\begin{array}{l}\text { Naves [48] } \\
2005 \text { Spain }\end{array}$ & $\begin{array}{l}(50+\text { yo), randomly } \\
\text { selected from the } \\
\text { Oviedo municipal } \\
\text { register. Postal } \\
\text { questionnaire + } \\
\text { examination }\end{array}$ & 229 & 229 & 0 & 74 & $\begin{array}{l}\text { The Int. Society } \\
\text { of Clinical } \\
\text { Densitometry: } \\
\text { BMD with a } \\
\text { T-score }-2.5 \\
\text { SD or less }\end{array}$ & $\begin{array}{l}\text { Hologic DXA, } \\
\text { QDR } 1000 \\
\text { densitometer }\end{array}$ & Point & $80+$ & 12.5 & & & $U$ \\
\hline \multirow{4}{*}{$\begin{array}{l}\text { Lumbar } \\
\text { spine } \\
\text { or hip }\end{array}$} & Sanfélix- & 2006-7, (50+ yo), & 824 & 0 & 824 & 47 & WHO BMD & Norland \& & Point & $60-64$ & & $22.5(16.3-28.8)$ & & U \\
\hline & $\begin{array}{l}\text { Genoves [4] } \\
2010 \text { Spain }\end{array}$ & strample of women & & & & & $\begin{array}{l}1-5 c o r e-2.5 \\
\text { SD or less }\end{array}$ & $\begin{array}{l}\text { Hologic } \\
\text { densitometer }\end{array}$ & & $65-69$ & & $32.4(25.2-39.4)$ & & \\
\hline & & included in the & & & & & & & & 70-74 & & $39.9(31.8-47.9)$ & & \\
\hline & & $\begin{array}{l}\text { Information } \\
\text { System of the } \\
\text { Valencia Healthcare } \\
\text { Agency, Valencia } \\
\text { (the FRAVO Study). } \\
\text { Interview, } \\
\text { questionnaire + } \\
\text { examination }\end{array}$ & & & & & & & & $75+$ & & $49.3(37.4-61.2)$ & & \\
\hline Lumbar & Vestergaard [52] & 1995-9, all in- and & 11359 & 1426 & 9933 & N/A & WHO BMD & The National & Point & $60-64$ & 14.7 & & 29.6 & L \\
\hline $\begin{array}{l}\text { Spine } \\
\text { or hip }\end{array}$ & 2005 Denmark & $\begin{array}{l}\text { outpatients recorded } \\
\text { in The National }\end{array}$ & & & & & $\begin{array}{l}\text { I-score }-2.5 \\
\text { SD or less }\end{array}$ & $\begin{array}{l}\text { Hospital } \\
\text { Discharge }\end{array}$ & & $65-69$ & 19.9 & & 44.0 & \\
\hline & & Hospital Discharge & & & & & & Register & & 70-74 & 26.1 & & 59.1 & \\
\hline & & based on all ICD-10 & & & & & & & & $75-79$ & 33.1 & & 72.2 & \\
\hline & & codes on OP. & & & & & & & & 80-84 & 40.4 & & 81.3 & \\
\hline & & & & & & & & & & $85-89$ & 47.8 & & 85.8 & \\
\hline & & & & & & & & & & $90-94$ & 55.3 & & 88.6 & \\
\hline & & & & & & & & & & $95+$ & 64.3 & & 92.3 & \\
\hline
\end{tabular}


Table 6 Description of studies on osteoporosis (OP) (Continued)

\begin{tabular}{|c|c|c|c|c|c|c|c|c|c|c|c|c|c|c|}
\hline \multirow{2}{*}{$\begin{array}{l}\text { Lumbar } \\
\text { spine }\end{array}$} & \multirow{2}{*}{$\begin{array}{l}\text { Cui [44] } 2008 \\
\text { South Korea }\end{array}$} & \multirow[b]{2}{*}{$\begin{array}{l}2004-5,(20-79 \text { yo), } \\
\text { from the Namwon } \\
\text { study and the } \\
\text { Thyroid Disease } \\
\text { Prevalence study } \\
\text { and from two } \\
\text { provinces. } \\
\text { Interview + } \\
\text { questionnaire + } \\
\text { clinical examination }\end{array}$} & \multirow[t]{2}{*}{4148} & \multirow[t]{2}{*}{1810} & \multirow[t]{2}{*}{2338} & \multirow[t]{2}{*}{39} & \multirow{2}{*}{$\begin{array}{l}\text { WHO BMD } \\
\text { T-score }-2.5 \\
\text { SD or less }\end{array}$} & \multirow[t]{2}{*}{ Lunar DXA } & \multirow[t]{2}{*}{ Point } & $60-69$ & 8.7 & \multicolumn{2}{|l|}{51.3} & \multirow[t]{2}{*}{ U } \\
\hline & & & & & & & & & & 70-79 & 12.8 & 60.2 & & \\
\hline \multirow{4}{*}{$\begin{array}{l}\text { Lumbar } \\
\text { spine }\end{array}$} & \multirow{4}{*}{$\begin{array}{l}\text { Henry [45] } \\
2000 \text { Australia }\end{array}$} & \multirow{4}{*}{$\begin{array}{l}\text { 1997, (20-94 yo), } \\
\text { age-stratified, } \\
\text { random, population- } \\
\text { based sample of } \\
\text { women registered } \\
\text { (compulsory) in the } \\
\text { Commonwealth of } \\
\text { Australia Electoral } \\
\text { Rolls, Geelong. } \\
\text { Questionnaire + } \\
\text { examination. }\end{array}$} & \multirow[t]{4}{*}{1494} & \multirow[t]{4}{*}{0} & \multirow[t]{4}{*}{1494} & \multirow[t]{4}{*}{63} & \multirow{4}{*}{$\begin{array}{l}\text { WHO BMD } \\
\text { T-score }-2.5 \\
\text { SD or less }\end{array}$} & \multirow{4}{*}{$\begin{array}{l}\text { Lunar DXA, } \\
\text { DPX-L } \\
\text { densitometer }\end{array}$} & \multirow[t]{4}{*}{ Point } & $60-64$ & & 10.5 & & \multirow[t]{4}{*}{ U } \\
\hline & & & & & & & & & & $65-69$ & & 15.2 & & \\
\hline & & & & & & & & & & $70-79$ & & 28.8 & & \\
\hline & & & & & & & & & & $80+$ & & & & \\
\hline \multirow{4}{*}{$\begin{array}{l}\text { Lumbar } \\
\text { spine }\end{array}$} & \multirow{4}{*}{$\begin{array}{l}\text { Sanfélix- } \\
\text { Genovés [49] } \\
2010 \text { Spain }\end{array}$} & \multirow{4}{*}{$\begin{array}{l}\text { 2006-7, (50+ yo), } \\
\text { stratified random } \\
\text { sample of women } \\
\text { included in the } \\
\text { Population } \\
\text { Information } \\
\text { System of the } \\
\text { Valencia Healthcare } \\
\text { Agency, Valencia } \\
\text { (the FRAVO Study). } \\
\text { Interview, } \\
\text { questionnaire + } \\
\text { examination }\end{array}$} & \multirow[t]{4}{*}{824} & \multirow[t]{4}{*}{0} & \multirow[t]{4}{*}{824} & \multirow[t]{4}{*}{47} & \multirow{4}{*}{$\begin{array}{l}\text { WHO BMD } \\
\text { T-score }-2.5 \\
\text { SD or less }\end{array}$} & \multirow{4}{*}{$\begin{array}{l}\text { Norland \& } \\
\text { Hologic } \\
\text { densitometer }\end{array}$} & \multirow[t]{4}{*}{ Point } & $60-64$ & & $18.5(12.7-24.3)$ & & \multirow[t]{4}{*}{ U } \\
\hline & & & & & & & & & & $65-69$ & & $28.2(21.4-35.0)$ & & \\
\hline & & & & & & & & & & $70-74$ & & $37.8(28.8-45.7)$ & & \\
\hline & & & & & & & & & & $75+$ & & $39.1(27.5-50.7)$ & & \\
\hline \multirow{2}{*}{$\begin{array}{l}\text { Lumbar } \\
\text { spine }\end{array}$} & Shin [50] 2010 & 2006-7, (40+ yo), & 3538 & 1547 & 1991 & 71 & WHO BMD & Lunar Prodigy & Point & $60-69$ & 13.7 & 28.5 & & $U$ \\
\hline & south Korea & $\begin{array}{l}\text { selected group trom } \\
\text { the } 2001 \text { cohort of } \\
\text { residents in the } \\
\text { farming community } \\
\text { of Ansung through } \\
\text { mailing, door- } \\
\text { to-door } \\
\text { and telehpone } \\
\text { solicitations (the } \\
\text { Korean Health and } \\
\text { Genome Study, } \\
\text { KHGS). Examination }\end{array}$ & & & & & $\begin{array}{l}\text { 1-score }-2.5 \\
\text { SD or less }\end{array}$ & & & $70-79$ & 22.4 & 47.5 & & \\
\hline Lumbar & Vestergaard [52] & 1995-9, all in- and & 11359 & 1426 & 9933 & N/A & WHO BMD & The National & Point & $60-64$ & 3.4 & & 17.3 & L \\
\hline spine & 2005 Denmark & $\begin{array}{l}\text { outpatients recorded } \\
\text { in The National }\end{array}$ & & & & & $\begin{array}{l}\text { T-score }-2.5 \\
\text { SD or less }\end{array}$ & $\begin{array}{l}\text { Hospital Discharge } \\
\text { Register }\end{array}$ & & $65-69$ & 4.6 & & 27.7 & \\
\hline
\end{tabular}


Table 6 Description of studies on osteoporosis (OP) (Continued)

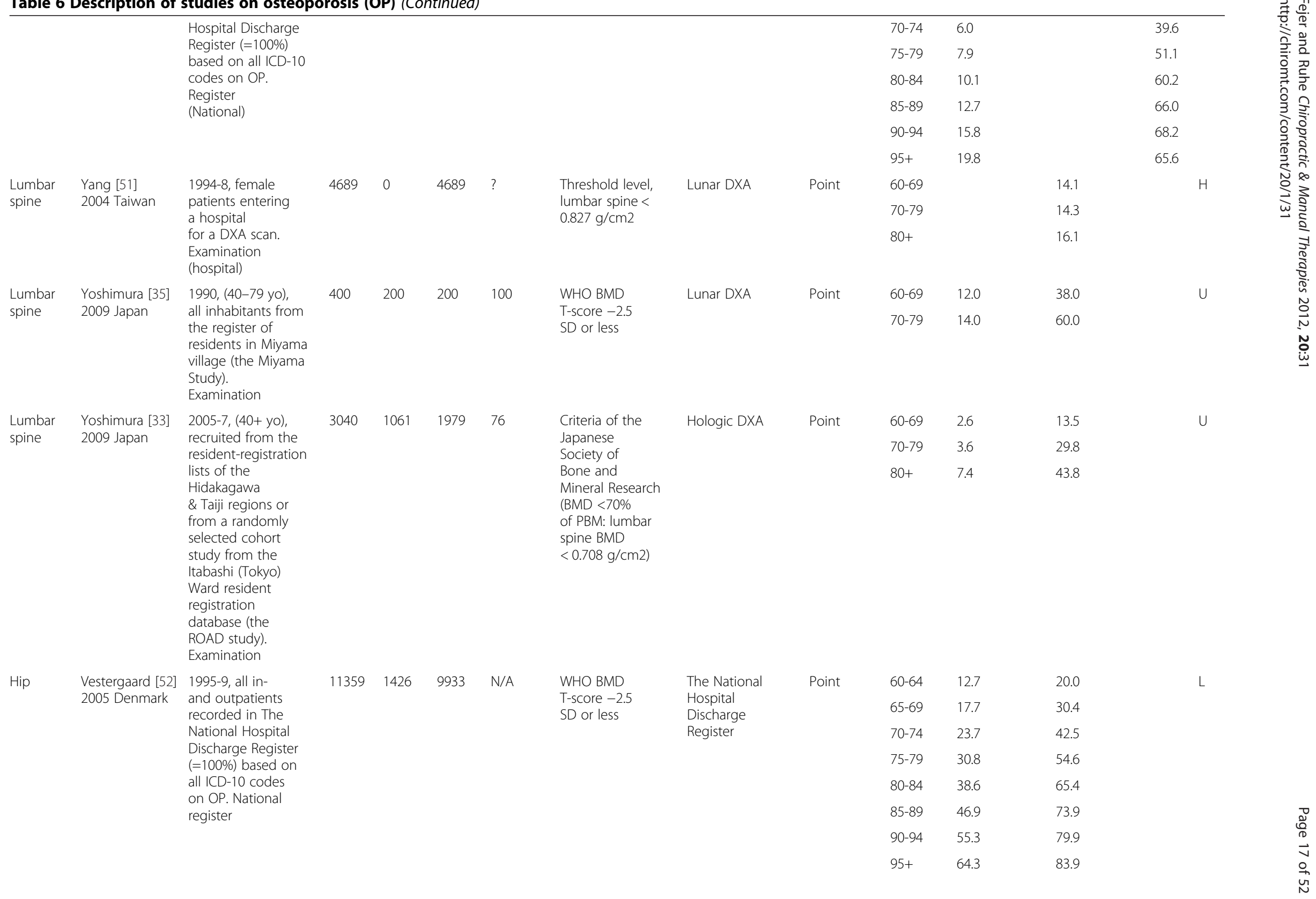


Table 6 Description of studies on osteoporosis (OP) (Continued)

\begin{tabular}{|c|c|c|c|c|c|c|c|c|c|c|c|c|c|}
\hline \multirow{2}{*}{$\begin{array}{l}\text { Femoral } \\
\text { neck }\end{array}$} & \multirow{2}{*}{$\begin{array}{l}\text { Cui [44] } 2008 \\
\text { South Korea }\end{array}$} & \multirow[b]{2}{*}{$\begin{array}{l}\text { 2004-5, (20-79 yo), } \\
\text { from the Namwon } \\
\text { study and the } \\
\text { Thyroid Disease } \\
\text { Prevalence study } \\
\text { invited to clinical } \\
\text { examination and } \\
\text { interview, from two } \\
\text { provinces. Interview, } \\
\text { questionnaire + } \\
\text { examination }\end{array}$} & \multirow[t]{2}{*}{4148} & \multirow[t]{2}{*}{1810} & \multirow[t]{2}{*}{2338} & \multirow[t]{2}{*}{39} & \multirow{2}{*}{$\begin{array}{l}\text { WHO BMD } \\
\text { T-score }-2.5 \\
\text { SD or less }\end{array}$} & \multirow[t]{2}{*}{ Lunar DXA } & \multirow[t]{2}{*}{ Point } & $60-69$ & 7.3 & 11.4 & \multirow[t]{2}{*}{ U } \\
\hline & & & & & & & & & & 70-79 & 15.2 & 36.7 & \\
\hline \multirow{4}{*}{$\begin{array}{l}\text { Femoral } \\
\text { neck }\end{array}$} & \multirow{4}{*}{$\begin{array}{l}\text { Henry [45] } \\
2000 \text { Australia }\end{array}$} & \multirow{4}{*}{$\begin{array}{l}\text { 1997, (20-94 yo), } \\
\text { age-stratified, } \\
\text { random, population- } \\
\text { based sample of } \\
\text { women registered } \\
\text { (compulsory) in the } \\
\text { Commonwealth of } \\
\text { Australia Electoral } \\
\text { Rolls, Geelong. } \\
\text { Questionnaire + } \\
\text { examination }\end{array}$} & \multirow[t]{4}{*}{1494} & \multirow[t]{4}{*}{0} & \multirow[t]{4}{*}{1494} & \multirow[t]{4}{*}{63} & \multirow{4}{*}{$\begin{array}{l}\text { WHO BMD } \\
\text { T-score - } 2.5 \\
\text { SD (NB. Hip: } \\
\text { femoral neck } \\
\text { used in this } \\
\text { review) }\end{array}$} & \multirow{4}{*}{$\begin{array}{l}\text { Lunar DXA, } \\
\text { DPX-L } \\
\text { densitometer }\end{array}$} & \multirow[t]{4}{*}{ Point } & $60-64$ & & 15.2 & \multirow[t]{4}{*}{$U$} \\
\hline & & & & & & & & & & $65-69$ & & 20.8 & \\
\hline & & & & & & & & & & 70-79 & & 31.6 & \\
\hline & & & & & & & & & & $80+$ & & 36.5 & \\
\hline $\begin{array}{l}\text { Femoral } \\
\text { neck }\end{array}$ & $\begin{array}{l}\text { Holt [46] } \\
2002 \text { UK }\end{array}$ & $\begin{array}{l}(50+\text { yo), random } \\
\text { sample from seven } \\
\text { health centres } \\
\text { (Aberdeen, Bath, } \\
\text { rural Cambridgeshire, } \\
\text { Harrow, Truro, } \\
\text { Norfolk, and } \\
\text { Cambridge City). } \\
\text { Questionnaire + } \\
\text { examination }\end{array}$ & 7426 & 2253 & 5173 & 48 & $\begin{array}{l}\text { WHO BMD } \\
\text { T-score - } 2.5 \\
\text { SD (NB. Hip: } \\
\text { femoral neck } \\
\text { used in this } \\
\text { review) }\end{array}$ & $\begin{array}{l}\text { Hologic DXA, } \\
\text { QDR } 1000 \\
\text { densitometer }\end{array}$ & Point & $65+$ & 2.7 & 8.1 & U \\
\hline \multirow{4}{*}{$\begin{array}{l}\text { Femoral } \\
\text { neck }\end{array}$} & \multirow{4}{*}{$\begin{array}{l}\text { Sanfélix- } \\
\text { Genovés [49] } \\
2010 \text { Spain }\end{array}$} & \multirow{4}{*}{$\begin{array}{l}\text { 2006-7, (50+ yo), } \\
\text { stratified random } \\
\text { sample of women } \\
\text { included in the } \\
\text { Population } \\
\text { Information } \\
\text { System of the } \\
\text { Valencia } \\
\text { Healthcare Agency, } \\
\text { Valencia (the FRAVO } \\
\text { Study). Interview, } \\
\text { questionnaire + } \\
\text { examination }\end{array}$} & \multirow[t]{4}{*}{824} & \multirow[t]{4}{*}{0} & \multirow[t]{4}{*}{824} & \multirow[t]{4}{*}{47} & \multirow{4}{*}{$\begin{array}{l}\text { WHO BMD } \\
\text { T-score }-2.5 \\
\text { SD or less }\end{array}$} & \multirow{4}{*}{$\begin{array}{l}\text { Norland \& } \\
\text { Hologic } \\
\text { Densitometer. }\end{array}$} & \multirow[t]{4}{*}{ Point } & $60-64$ & $6.9(3.1-10.7)$ & & \multirow[t]{4}{*}{$U$} \\
\hline & & & & & & & & & & $65-69$ & $10.1(9.4-21.3)$ & & \\
\hline & & & & & & & & & & 70-74 & $15.4(9.4-21.3)$ & & \\
\hline & & & & & & & & & & $75+$ & $34.8(23.4-46.1)$ & & \\
\hline
\end{tabular}


Table 6 Description of studies on osteoporosis (OP) (Continued)

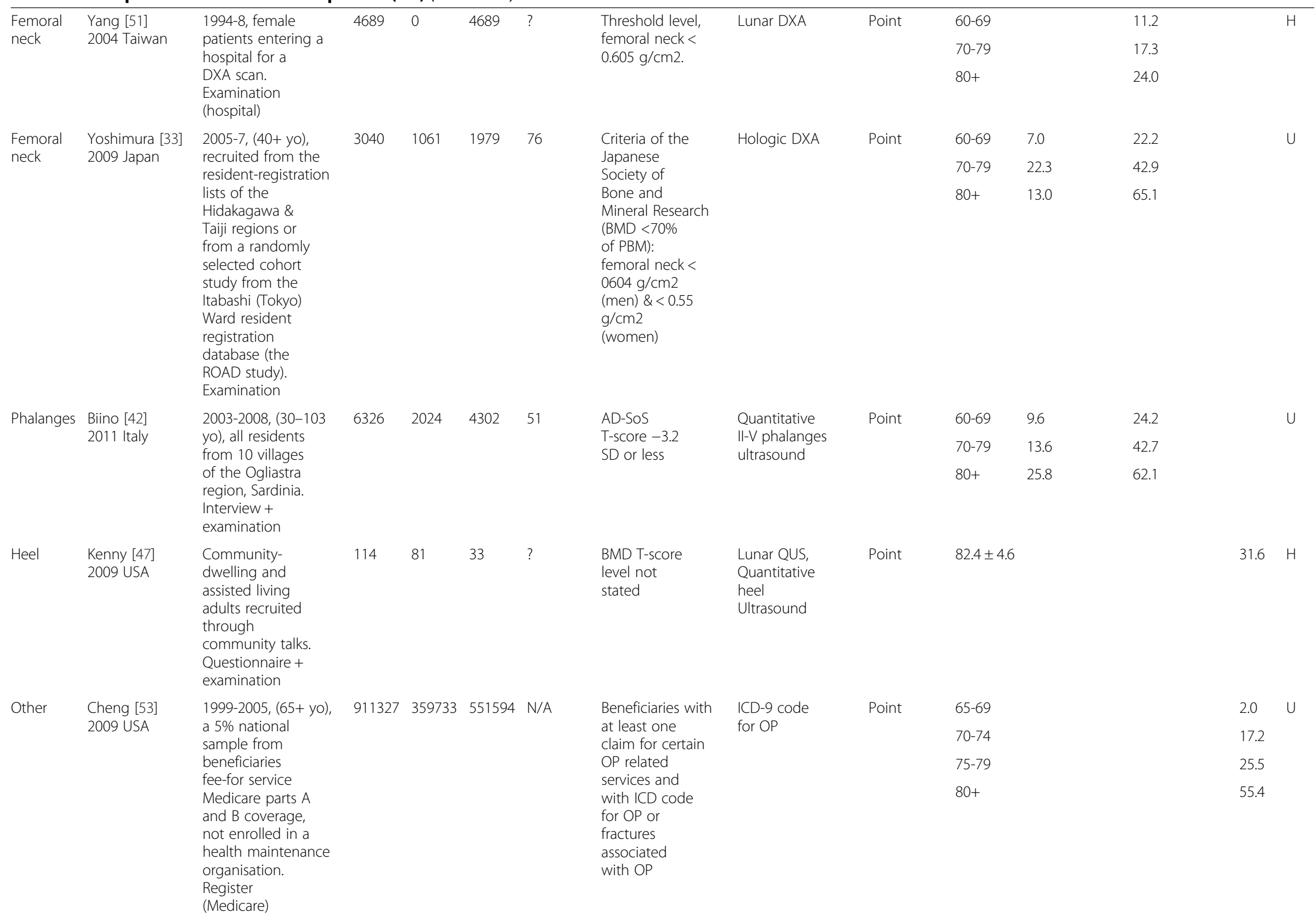




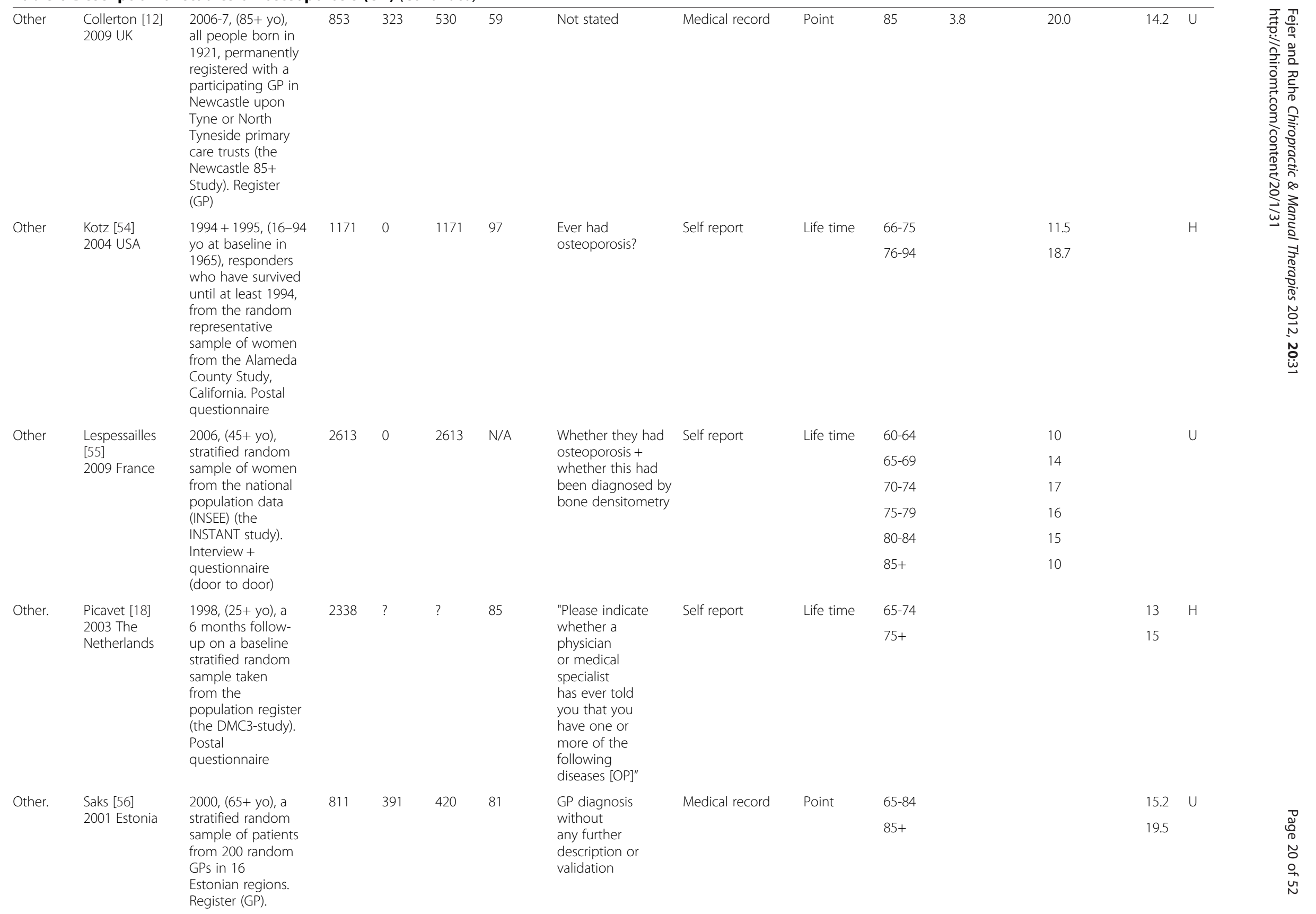


Table 6 Description of studies on osteoporosis (OP) (Continued)

\begin{tabular}{|c|c|c|c|c|c|c|c|c|c|c|c|c|c|c|}
\hline \multirow[t]{3}{*}{ Other } & \multirow{3}{*}{$\begin{array}{l}\text { Werner [57] } \\
2003 \text { Israel }\end{array}$} & \multirow[b]{3}{*}{$\begin{array}{l}\text { 1997-8, (60+ yo), a } \\
\text { stratified random } \\
\text { sample of Jewish } \\
\text { and Arab } \\
\text { community- } \\
\text { dwelling persons } \\
\text { from the Central } \\
\text { Bureau of Statistics } \\
\text { (the Israeli Survey } \\
\text { of Elderly Persons } \\
\text { Aged } 60 \text { and } \\
\text { Over Study). } \\
\text { Interview + } \\
\text { questionnaire } \\
\text { (home visit) }\end{array}$} & \multirow[t]{3}{*}{3022} & \multirow{3}{*}{\multicolumn{2}{|c|}{1688}} & \multirow[t]{3}{*}{1334} & \multirow[t]{3}{*}{60} & \multirow{3}{*}{$\begin{array}{l}\text { Whether a } \\
\text { physician } \\
\text { had ever } \\
\text { diagnosed } \\
\text { them as having } \\
\text { osteoporosis }\end{array}$} & \multirow[t]{3}{*}{ Self report } & \multirow[t]{3}{*}{ Life time } & $60-69$ & 5.0 & 20.5 & \multirow[t]{3}{*}{ U } \\
\hline & & & & & & & & & & & 70-79 & 8.0 & 26.3 & \\
\hline & & & & & & & & & & & $80+$ & 7.9 & 28.3 & \\
\hline
\end{tabular}

*Prevalence estimates without decimals are obtained from figures/graphs in the article and should be interpreted with caution.

R: Register. L: Low, U: Unclear, H: High.

BMD: Bone mineral density; WHO: World Health Organization, GP: general practitioner, DXA: Dual X-ray absorptometry 
Table 7 Description of back pain (BP) and neck pain (NP)

\begin{tabular}{|c|c|c|c|c|c|c|c|c|c|c|c|c|c|c|}
\hline & \multirow{2}{*}{$\begin{array}{l}\text { First author } \\
\text { Publ. year } \\
\text { Country }\end{array}$} & \multirow{2}{*}{$\begin{array}{l}\text { Study design / } \\
\text { Population / Method } \\
\text { of collection }\end{array}$} & \multicolumn{3}{|c|}{ Sample size } & \multirow{2}{*}{$\begin{array}{l}\text { Crude } \\
\text { response } \\
\text { rate }(\%)\end{array}$} & \multirow{2}{*}{$\begin{array}{l}\text { Outcome } \\
\text { definition }\end{array}$} & \multirow{2}{*}{$\begin{array}{l}\text { Outcome } \\
\text { assessment } \\
\text { method }\end{array}$} & \multirow{2}{*}{$\begin{array}{l}\text { Prevalence } \\
\text { period }\end{array}$} & \multirow[t]{2}{*}{ Age } & \multicolumn{3}{|c|}{ Prevalence* $(95 \% \mathrm{Cl})$} & \multirow{2}{*}{$\begin{array}{l}\text { Risk } \\
\text { of } \\
\text { bias }\end{array}$} \\
\hline & & & Total & $M$ & $F$ & & & & & & $M$ & $F$ & Total & \\
\hline \multirow[t]{2}{*}{ LBP } & \multirow{2}{*}{$\begin{array}{l}\text { Andrianakos } \\
\text { [41] } 2006 \\
\text { Greece }\end{array}$} & \multirow[b]{2}{*}{$\begin{array}{l}\text { 1966-99, (19+ yo), } \\
\text { the total population } \\
\text { in } 7 \text { mixed } \\
\text { communities + } \\
\text { random sample } \\
\text { in another } 2 \\
\text { mixed communities. } \\
\text { Interview, } \\
\text { questionnaire + } \\
\text { examination } \\
\text { (home visit, } \\
\text { rheumatologist) }\end{array}$} & \multirow[t]{2}{*}{8740} & \multirow[t]{2}{*}{4269} & \multirow[t]{2}{*}{4471} & \multirow[t]{2}{*}{82} & \multirow[b]{2}{*}{$\begin{array}{l}\text { LBP localized in } \\
\text { the back area } \\
\text { between the lower } \\
\text { limits of the chest } \\
\text { and the gluteal folds, } \\
\text { either radiating or } \\
\text { not along a lower } \\
\text { extremity. Past LBP } \\
\text { included if recurrent } \\
\text { and chronic causes }\end{array}$} & Self report & Life time & $59-64$ & & & 18 & L \\
\hline & & & & & & & & & & $69+$ & & & 19 & \\
\hline \multirow[t]{2}{*}{ LBP } & \multirow{2}{*}{$\begin{array}{l}\text { Salaffi [79] } \\
2005 \text { Italy }\end{array}$} & \multirow[b]{2}{*}{$\begin{array}{l}2004,(18+\text { yo), } \\
\text { stratified randomised } \\
\text { sample selected } \\
\text { from the practice } \\
\text { lists of } 16 \text { general } \\
\text { practitioner-GPs } \\
\text { representative of the } \\
\text { practices in the } \\
\text { Marches, central } \\
\text { Italy. Postal } \\
\text { questionnaire }\end{array}$} & \multirow[t]{2}{*}{2155} & \multirow[t]{2}{*}{$?$} & \multirow[t]{2}{*}{$?$} & \multirow[t]{2}{*}{54} & \multirow[b]{2}{*}{$\begin{array}{l}\text { LBP defined as pain } \\
\text { localized in the back } \\
\text { area between the } \\
\text { lower limits of the } \\
\text { chest and the gluteal } \\
\text { folds, either radiating } \\
\text { or not along a } \\
\text { lower extremity. } \\
\text { Three satisfactory } \\
\text { screening criteria: } \\
\text { I) Report of ever } \\
\text { having had LBP, } \\
\text { II) A health care } \\
\text { provider visit for LBP } \\
\text { in the previous six } \\
\text { months, and } \\
\text { III) LBP that began } \\
\text { more than } 3 \text { months } \\
\text { previously }\end{array}$} & Self report & Life time & $65-74$ & & & 29 & U \\
\hline & & & & & & & & & & $75+$ & & & 26 & \\
\hline \multirow[t]{3}{*}{ LBP } & \multirow{3}{*}{$\begin{array}{l}\text { Cecchi [60] } \\
2006 \text { Italy }\end{array}$} & \multirow{3}{*}{$\begin{array}{l}\text { 1998-2000, }(65+), \text { a } \\
\text { representative cohort } \\
\text { was selected from } \\
\text { the registries of } \\
\text { Greve in Chianti } \\
\text { (rural area) and } \\
\text { Bagno a Ripoli } \\
\text { (urban area near } \\
\text { Florence). } \\
\text { Interview, } \\
\text { questionnaire + } \\
\text { examination } \\
\text { (home visit, } \\
\text { rheumatologist). }\end{array}$} & \multirow[t]{3}{*}{1008} & \multirow[t]{3}{*}{443} & \multirow[t]{3}{*}{565} & \multirow[t]{3}{*}{80} & \multirow{3}{*}{$\begin{array}{l}\text { Any frequent BP } \\
\text { episodes (defined as } \\
\text { quite often-almost } \\
\text { every day) over the } \\
\text { past } 12 \text { months }\end{array}$} & \multirow[t]{3}{*}{ Self report } & \multirow[t]{3}{*}{ One year } & $65-74$ & 20.7 & 38.1 & & U \\
\hline & & & & & & & & & & $75-84$ & 26.3 & 44.4 & & \\
\hline & & & & & & & & & & $85+$ & 25.0 & 25.0 & & \\
\hline
\end{tabular}


Table 7 Description of back pain (BP) and neck pain (NP) (Continued)

\begin{tabular}{|c|c|c|c|c|c|c|c|c|c|c|c|c|c|c|}
\hline LBP & $\begin{array}{l}\text { Hartvigsen [69] } \\
2006 \text { Denmark }\end{array}$ & $\begin{array}{l}\text { 2003, (70-102 yo), } \\
\text { twins from the } \\
\text { populations-based } \\
\text { twin study } \\
\text { (LSADT). } \\
\text { Interview + } \\
\text { questionnaire } \\
\text { (home) }\end{array}$ & 1844 & $?$ & $?$ & 84 & $\begin{array}{l}\text { Modified version of } \\
\text { the standardised } \\
\text { Nordic } \\
\text { Questionnaire } \\
\text { (SNQ) on } \\
\text { Musculoskeletal } \\
\text { Pain }\end{array}$ & $\begin{array}{l}\text { Self } \\
\text { report }\end{array}$ & One year & $72-102$ & $\begin{array}{l}21 \\
(19-23)\end{array}$ & $\begin{array}{l}32 \\
(29-35)\end{array}$ & & U \\
\hline \multirow[t]{3}{*}{ LBP } & \multirow{3}{*}{$\begin{array}{l}\text { Hicks [71] } \\
2008 \text { USA }\end{array}$} & \multirow{3}{*}{$\begin{array}{l}(62+\text { yo), community- } \\
\text { dwellers from } 4 \\
\text { retirement } \\
\text { communities (The } \\
\text { Retirement } \\
\text { Community Back } \\
\text { Pain Study). } \\
\text { Postal } \\
\text { questionnaire }\end{array}$} & \multirow[t]{3}{*}{522} & \multirow[t]{3}{*}{170} & \multirow[t]{3}{*}{352} & \multirow[t]{3}{*}{52} & \multirow{3}{*}{$\begin{array}{l}\text { "In the past year, } \\
\text { have you had } \\
\text { any low } \\
\text { back pain? If yes, } \\
\text { please rate your } \\
\text { usual back pain } \\
\text { over the past year } \\
\text { on a scale from } \\
0 \text { to } 10 \text { " }\end{array}$} & \multirow{3}{*}{$\begin{array}{l}\text { Self } \\
\text { report }\end{array}$} & \multirow[t]{3}{*}{ One year } & $60-69$ & & & 26.7 & \multirow[t]{3}{*}{ U } \\
\hline & & & & & & & & & & $70-79$ & & & 30.5 & \\
\hline & & & & & & & & & & $80+$ & & & 24.8 & \\
\hline \multirow[t]{2}{*}{ LBP } & \multirow{2}{*}{$\begin{array}{l}\text { Picavet [78] } \\
2003 \text { The } \\
\text { Netherlands }\end{array}$} & \multirow[b]{2}{*}{$\begin{array}{l}\text { 1998, }(25+\text { yo), } \\
\text { stratified random } \\
\text { sample taken } \\
\text { from the population } \\
\text { register (the } \\
\text { DMC3-study). } \\
\text { Postal } \\
\text { questionnaire }\end{array}$} & \multirow[t]{2}{*}{3664} & \multirow[t]{2}{*}{$45 \%$} & \multirow[t]{2}{*}{$55 \%$} & \multirow[t]{2}{*}{46} & \multirow{2}{*}{$\begin{array}{l}\text { "Did you have } \\
\text { pain [in the lower } \\
\text { part of the back] } \\
\text { during the past } \\
12 \text { months?" }\end{array}$} & \multirow{2}{*}{$\begin{array}{l}\text { Self } \\
\text { report }\end{array}$} & \multirow[t]{2}{*}{ One year } & $65-74$ & & & 48 & \multirow[t]{2}{*}{ U } \\
\hline & & & & & & & & & & $75+$ & & & 32 & \\
\hline LBP & $\begin{array}{l}\text { Santos- } \\
\text { Eggimann } \\
\text { [80] } 2000 \\
\text { Switzerland }\end{array}$ & $\begin{array}{l}\text { 1992-3, (25-74 yo), } \\
\text { two-stage probabilistic } \\
\text { stratified random } \\
\text { sample of inhabitants } \\
\text { from the population } \\
\text { files of the Vaud- } \\
\text { Fribourg \& Ticino } \\
\text { communes (the } \\
\text { WHO MONICA } \\
\text { study). Questionnaire } \\
\text { (postal)+ } \\
\text { examination. }\end{array}$ & 3227 & $?$ & $?$ & 61 & $\begin{array}{l}\text { The Standardized } \\
\text { Nordic } \\
\text { Questionnaire: } \\
\text { any ache, pain, or } \\
\text { discomfort located } \\
\text { in the lower back } \\
\text { (indicated by the } \\
\text { shaded area on a } \\
\text { diagram), with or } \\
\text { without radiation } \\
\text { to one or both } \\
\text { legs (sciatica) the } \\
\text { preceding } 12 \text { months }\end{array}$ & $\begin{array}{l}\text { Self } \\
\text { report }\end{array}$ & $\begin{array}{l}\text { One year } \\
\text { (>7 days) }\end{array}$ & $65-74$ & 28.5 & 38.5 & & L \\
\hline LBP & $\begin{array}{l}\text { Goubert [66] } \\
2004 \text { Belgium }\end{array}$ & $\begin{array}{l}\text { 2001, (17+ yo), a } \\
\text { representative access } \\
\text { panel of individuals } \\
\text { who regularly } \\
\text { participate in postal } \\
\text { surveys. Postal } \\
\text { questionnaire }\end{array}$ & 1624 & $?$ & $?$ & 65 & $\begin{array}{l}\text { Participants indicated } \\
\text { whether they had } \\
\text { experienced LBP } \\
\text { pain in the past } \\
\text { six months (The } \\
\text { Graded Chronic } \\
\text { Pain Scale) }\end{array}$ & $\begin{array}{l}\text { Self } \\
\text { report }\end{array}$ & $\begin{array}{l}\text { Six } \\
\text { months }\end{array}$ & $65+$ & & & 36.7 & U \\
\hline \multirow[t]{3}{*}{ LBP } & \multirow{3}{*}{$\begin{array}{l}\text { Miro [75] } \\
2007 \text { Spain }\end{array}$} & \multirow{3}{*}{$\begin{array}{l}(65+\text { yo), stratified } \\
\text { random sample } \\
\text { taken from the } \\
\text { population census }\end{array}$} & \multirow[t]{3}{*}{592} & \multirow[t]{3}{*}{274} & \multirow[t]{3}{*}{318} & \multirow[t]{3}{*}{99} & \multirow{3}{*}{$\begin{array}{l}\text { The Chronic Pain } \\
\text { Grade: "In the past } \\
3 \text { months have you } \\
\text { had pain that has }\end{array}$} & \multirow{3}{*}{$\begin{array}{l}\text { Self } \\
\text { report }\end{array}$} & \multirow{3}{*}{$\begin{array}{l}\text { Three } \\
\text { months }\end{array}$} & $65-74$ & & & 61.0 & \multirow[t]{3}{*}{ L } \\
\hline & & & & & & & & & & $75-84$ & & & 62.6 & \\
\hline & & & & & & & & & & $85+$ & & & 44.2 & \\
\hline
\end{tabular}


Table 7 Description of back pain (BP) and neck pain (NP) (Continued)

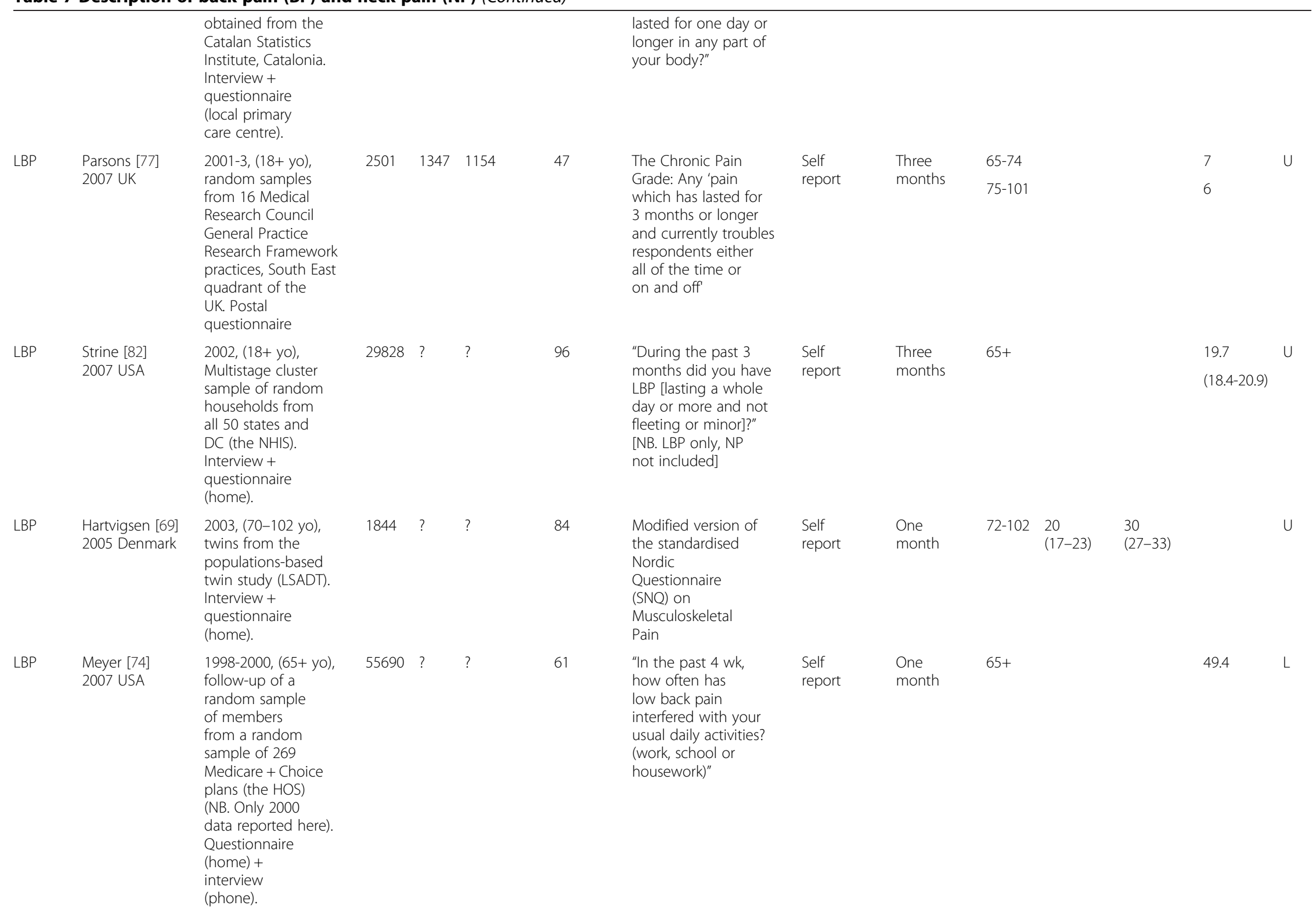


Table 7 Description of back pain (BP) and neck pain (NP) (Continued)

\begin{tabular}{|c|c|c|c|c|c|c|c|c|c|c|c|c|c|c|}
\hline LBP & $\begin{array}{l}\text { Stranjalis [81] } \\
2004 \text { Greece }\end{array}$ & $\begin{array}{l}2000,(15+\text { yo), a } \\
2000 \text { person sample, } \\
\text { selected via a } \\
\text { multi-stage sampling } \\
\text { of rural, semi-urban } \\
\text { and urban residents } \\
\text { through "random } \\
\text { numbers" of starting } \\
\text { points followed by } \\
\text { "statistical step of } \\
\text { five" in } 47 \text { cities, } \\
\text { towns or villages } \\
\text { (until reaching a } \\
\text { total of } 2000 \\
\text { persons). } \\
\text { Interview + } \\
\text { questionnaire } \\
\text { (home). }\end{array}$ & 1846 & $?$ & $?$ & 92 & $\begin{array}{l}\text { "Did you have low } \\
\text { back pain during } \\
\text { the last month?" }\end{array}$ & $\begin{array}{l}\text { Self } \\
\text { report }\end{array}$ & One month & $65+$ & & & 46.9 & $\mathrm{H}$ \\
\hline LBP & $\begin{array}{l}\text { Suka [83] } \\
2009 \text { Japan }\end{array}$ & $\begin{array}{l}\text { 2005, About } 1000 \\
\text { persons from five } \\
\text { different healthcare } \\
\text { facilities were asked } \\
\text { to participate. } \\
\text { Questionnaire } \\
\text { (Health care } \\
\text { facility). }\end{array}$ & 5652 & $?$ & $?$ & $?$ & $\begin{array}{l}\text { Musculoskeletal pain } \\
\text { for more than } 1 \text { week } \\
\text { during the last } \\
\text { month (marked on } \\
\text { a drawing with } \\
\text { predefined body } \\
\text { regions) }\end{array}$ & $\begin{array}{l}\text { Self } \\
\text { report }\end{array}$ & $\begin{array}{l}\text { One } \\
\text { month }\end{array}$ & $60-69$ & 23.8 & 23.2 & & $\mathrm{H}$ \\
\hline \multirow[t]{3}{*}{ LBP } & \multirow{3}{*}{$\begin{array}{l}\text { Thomas [87] } \\
2004 \text { UK }\end{array}$} & \multirow{3}{*}{$\begin{array}{l}\text { ( } 50+\text { yo), all patients } \\
\text { from three GPs } \\
\text { from the North } \\
\text { Staffordshire } \\
\text { Primary Care } \\
\text { Research Consortium } \\
\text { (the NorStOP). } \\
\text { Postal } \\
\text { questionnaire }\end{array}$} & \multirow[t]{3}{*}{7878} & \multirow[t]{3}{*}{$?$} & \multirow[t]{3}{*}{$?$} & \multirow[t]{3}{*}{70} & \multirow{3}{*}{$\begin{array}{l}\text { "In the past } 4 \text { weeks } \\
\text { have you had pain } \\
\text { that has lasted for } \\
\text { one day or longer } \\
\text { in any part of your } \\
\text { body?" [supplemented } \\
\text { by a full body } \\
\text { manikin] }\end{array}$} & \multirow{3}{*}{$\begin{array}{l}\text { Self } \\
\text { report }\end{array}$} & \multirow{3}{*}{$\begin{array}{l}\text { One } \\
\text { month }\end{array}$} & $60-69$ & & & 35.1 & U \\
\hline & & & & & & & & & & 70-79 & & & 29.9 & \\
\hline & & & & & & & & & & $80+$ & & & 27.3 & \\
\hline \multirow[t]{2}{*}{ LBP } & \multirow{2}{*}{$\begin{array}{l}\text { Webb [85] } \\
2003 \text { UK }\end{array}$} & \multirow{2}{*}{$\begin{array}{l}\text { (16+ yo), stratified } \\
\text { sample of patients } \\
\text { from three GP in } \\
\text { West Pennine, } \\
\text { East of Manchester. } \\
\text { Questionnaire. }\end{array}$} & \multirow[t]{2}{*}{4515} & \multirow[t]{2}{*}{$?$} & \multirow[t]{2}{*}{$?$} & \multirow[t]{2}{*}{78} & \multirow[b]{2}{*}{$\begin{array}{l}\text { Pain lasting for more } \\
\text { than } 1 \text { week, over } \\
\text { the last month, in } \\
\text { any of seven areas } \\
\text { (back, neck, shoulder, } \\
\text { elbow, hand, hip, } \\
\text { knee) or in } \\
\text { multiple joints }\end{array}$} & \multirow{2}{*}{$\begin{array}{l}\text { Self } \\
\text { report }\end{array}$} & \multirow{2}{*}{$\begin{array}{l}\text { One } \\
\text { month }\end{array}$} & $65-74$ & 20.6 & 32.1 & & U \\
\hline & & & & & & & & & & $75+$ & 17.4 & 30.9 & & \\
\hline LBP & $\begin{array}{l}\text { Yaron [86] } \\
2011 \text { Israel }\end{array}$ & $\begin{array}{l}\text { 2002, 2006, 2008, } \\
\text { (20+ yo), stratified } \\
\text { sample drawn from } \\
\text { a telephone database } \\
\text { on different } \\
\text { population sectors. }\end{array}$ & 2520 & $47 \%$ & $53 \%$ & $59-66$ & $\begin{array}{l}\text { The Community } \\
\text { Oriented Program } \\
\text { for the Control of } \\
\text { Rheumatic Diseases } \\
\text { core questionnaire } \\
\text { (CCQ): "In the past } 7\end{array}$ & Self report & One week & $61+$ & & & 67.2 & U \\
\hline
\end{tabular}


Table 7 Description of back pain (BP) and neck pain (NP) (Continued)

\begin{tabular}{|c|c|c|c|}
\hline $\begin{array}{l}\text { Telephone } \\
\text { interview + } \\
\text { questionnaire }\end{array}$ & & & \\
\hline $\begin{array}{l}\text { 2005-06, (65+ yo), } \\
\text { residents of } \\
\text { Seongnam City. } \\
\text { Questionnaire + } \\
\text { examination } \\
\text { (hospital). }\end{array}$ & 714 & 299 & 415 \\
\hline
\end{tabular}

days have you

experienced pain

in any of the

following sites:

[ankles]?"

$\begin{array}{ll}\text { Baek [59] } & \text { 2005-06, (65+ yo), } \\ 2010 & \text { residents of } \\ \text { South Korea } & \text { Seongnam City. } \\ & \begin{array}{l}\text { Questionnaire + } \\ \text { examination } \\ \text { (hospital). }\end{array}\end{array}$

64

moment"

LBP

$\begin{array}{ll}\text { Carmona [11] } & \text { (20+ yo), a stratified } \\ \text { 2001 Spain } & \text { multistage cluster } \\ & \text { sample from the } \\ & \text { censuses of } 20 \\ & \text { municipalities. } \\ \text { Questionnaire } & \text { (home) }+ \\ \text { interview } & \text { (rheumatologist) }\end{array}$

LBP

Freburger [64] 2009 USA

1992 + 2006, $(21+$ yo), $2723 \quad ? \quad$ ?
two-staged stratified
probability sample
of North Carolina
households with
telephone numbers
(NB only data from
2006 survey is
included).
Interview +
questionnaire
(phone).

$2192 \quad 1014 \quad 1178$

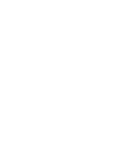

(rheumatologist).

$\begin{array}{lllll}\text { Picavet [78] } & \text { 1998, (25+ yo), } & 3664 & 45 \% & 55 \% \\ 2003 \text { The } & \text { stratified random } \\ \text { sample taken from } \\ \text { the population } \\ \text { register (the } \\ \text { DMC3-study). } \\ \text { Postal } \\ \text { questionnaire }\end{array}$

83
LBP defined as pain at the level of the
waist or below, with
or without buttock
and/or leg pain.
Chronic LBP:
1) pain and activity
limitations nearly
every day for the
past 3 months or
2) more than 24
episodes of pain
that limited activity
for 1 day or more

in the past year

[Lower part of the
back] pain during the survey
The Oswestry

Disability

Index on LBP:

"pain at the

Self report Point

(n)

understood by

ask about pain 
Table 7 Description of back pain (BP) and neck pain (NP) (Continued)

\begin{tabular}{|c|c|c|c|c|c|c|c|c|c|c|c|c|c|c|}
\hline $\mathrm{BP}$ & $\begin{array}{l}\text { Denard [62] } \\
2010 \text { UK }\end{array}$ & $\begin{array}{l}2000-2,(65+\text { yo), } \\
\text { a random sample } \\
\text { of } 300 \text { community } \\
\text { dwelling men } \\
\text { recruited at } 6 \text { US } \\
\text { academic medical } \\
\text { centers (The MrOS } \\
\text { cohort). } \\
\text { Questionnaire } \\
\text { (postal) + } \\
\text { examination. }\end{array}$ & 300 & 295 & 0 & 98 & $\begin{array}{l}\text { Any } \mathrm{BP} \text { in the } \\
\text { past } 12 \text { months }\end{array}$ & $\begin{array}{l}\text { Self } \\
\text { report }\end{array}$ & $\begin{array}{l}\text { One } \\
\text { year }\end{array}$ & $65+$ & 65 & & & U \\
\hline \multirow[t]{2}{*}{$\mathrm{BP}$} & \multirow[t]{2}{*}{$\begin{array}{l}\text { Keenan [73] } \\
2006 \text { UK }\end{array}$} & \multirow[t]{2}{*}{$\begin{array}{l}\text { 1993, (55+ yo), a } \\
\text { two-stage random } \\
\text { sample from the } \\
\text { North Yorkshire } \\
\text { Family Health } \\
\text { Services Authority. } \\
\text { Postal } \\
\text { questionnaire }\end{array}$} & \multirow[t]{2}{*}{16222} & \multirow[t]{2}{*}{$?$} & \multirow[t]{2}{*}{$?$} & \multirow[t]{2}{*}{86} & \multirow[t]{2}{*}{$\begin{array}{l}\text { Any swelling, pain, } \\
\text { or stiffness in any } \\
\text { of their joints that } \\
\text { lasted }>6 \text { weeks in } \\
\text { the previous } 3 \\
\text { months (identified } \\
\text { on a manikin) }\end{array}$} & \multirow[t]{2}{*}{$\begin{array}{l}\text { Self } \\
\text { report }\end{array}$} & \multirow[t]{2}{*}{$\begin{array}{l}\text { Three } \\
\text { months }\end{array}$} & $65-74$ & $\begin{array}{l}13.5 \\
(12.2-14.8)\end{array}$ & $\begin{array}{l}18.2 \\
(16.8-19.7)\end{array}$ & $\begin{array}{l}16.1 \\
(14.7-17.5)\end{array}$ & \multirow[t]{2}{*}{ L } \\
\hline & & & & & & & & & & $75+$ & $\begin{array}{l}11.4 \\
(10.2-12.6)\end{array}$ & $\begin{array}{l}19.0 \\
(17.6-20.5)\end{array}$ & $\begin{array}{l}16.4 \\
(15.1-17.8)\end{array}$ & \\
\hline \multirow[t]{4}{*}{$\mathrm{BP}$} & \multirow{4}{*}{$\begin{array}{l}\text { Hartvigsen [68] } \\
2004 \text { Denmark }\end{array}$} & \multirow{4}{*}{$\begin{array}{l}\text { 1995,1997,1999, } \\
\text { 2001, (70-102 yo), } \\
\text { twins from the } \\
\text { populations-based } \\
\text { twin study (LSADT). } \\
\text { Interview + } \\
\text { questionnaire } \\
\text { (home). }\end{array}$} & \multirow[t]{4}{*}{4484} & \multirow[t]{4}{*}{$?$} & \multirow[t]{4}{*}{$?$} & \multirow[t]{4}{*}{100} & \multirow{4}{*}{$\begin{array}{l}\text { "Have you during } \\
\text { the past month } \\
\text { suffered from pain } \\
\text { or stiffness in the } \\
\text { neck or shoulders?" + } \\
\text { diagnosis had } \\
\text { been made by a } \\
\text { physician }\end{array}$} & \multirow{4}{*}{$\begin{array}{l}\text { Self } \\
\text { report }\end{array}$} & \multirow{4}{*}{$\begin{array}{l}\text { One } \\
\text { month }\end{array}$} & $70-74$ & 14 & 18 & & \multirow[t]{4}{*}{ 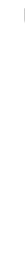 } \\
\hline & & & & & & & & & & 75-79 & 12 & 17 & & \\
\hline & & & & & & & & & & $80-84$ & 10 & 15 & & \\
\hline & & & & & & & & & & $85+$ & 11 & 16 & & \\
\hline $\mathrm{BP}$ & $\begin{array}{l}\text { Hartvigsen [70] } \\
2008 \text { Denmark }\end{array}$ & $\begin{array}{l}\text { 2005, (100 yo), all } \\
\text { Danes born in } 1905 \\
\text { were located } \\
\text { through the Danish } \\
\text { Civil Registration } \\
\text { System. } \\
\text { Interview + } \\
\text { questionnaire } \\
\text { (home). }\end{array}$ & 256 & $?$ & $?$ & 56 & $\begin{array}{l}\text { "During the past } \\
\text { month, have you } \\
\text { been suffering } \\
\text { from back pain, } \\
\text { acute back pain, } \\
\text { or lumbago?" }\end{array}$ & $\begin{array}{l}\text { Self } \\
\text { report }\end{array}$ & $\begin{array}{l}\text { One } \\
\text { month }\end{array}$ & 100 & 16.7 & 29.4 & 27.3 & U \\
\hline \multirow[t]{4}{*}{$\mathrm{BP}$} & \multirow{4}{*}{$\begin{array}{l}\text { Docking [63] } \\
2011 \text { UK }\end{array}$} & \multirow{4}{*}{$\begin{array}{l}\text { 1988-90, }(75+\text { yo), } \\
\text { original cohort from } \\
\text { the } 1985 \text { Cambridge } \\
\text { City over } 75 \mathrm{~s} \\
\text { Cohort Study } \\
\text { randomly chosen } \\
\text { from a selection of } \\
\text { geographically and } \\
\text { socially representative } \\
\text { general practices in } \\
\text { Cambridge. } \\
\text { Interview + } \\
\text { questionnaire } \\
\text { (home). }\end{array}$} & \multirow[t]{4}{*}{1174} & \multirow[t]{4}{*}{$35 \%$} & \multirow[t]{4}{*}{$65 \%$} & \multirow[t]{4}{*}{$45 \%$} & \multirow{4}{*}{$\begin{array}{l}\text { Have you recently } \\
\text { had an } \\
\text { illness or condition } \\
\text { which prevented you } \\
\text { carrying out normal } \\
\text { day to day routine? } \\
\text { [accompanied by a list } \\
\text { of conditions including } \\
\text { back pain]. (=Any } \\
\text { back pain) }\end{array}$} & \multirow[t]{4}{*}{ Self report } & \multirow[t]{4}{*}{ Point } & $77-79$ & & & 27.0 & \multirow[t]{4}{*}{$u$} \\
\hline & & & & & & & & & & 80-84 & & & 31.1 & \\
\hline & & & & & & & & & & 85-89 & & & 27.0 & \\
\hline & & & & & & & & & & $90-100$ & & & 29.1 & \\
\hline
\end{tabular}


Table 7 Description of back pain (BP) and neck pain (NP) (Continued)

\begin{tabular}{|c|c|c|c|c|c|c|c|c|c|c|c|c|c|c|}
\hline \multirow{2}{*}{ BP } & \multirow{2}{*}{$\begin{array}{l}\text { Jacobs [72] } \\
2006 \text { Jerusalem }\end{array}$} & \multirow[b]{2}{*}{$\begin{array}{l}1990 \text { \& 1998-9, } \\
\text { ( } 70 \& 77 \text { yo), recruited } \\
\text { from the electoral } \\
\text { register of the } \\
\text { Israeli Ministry of } \\
\text { Interior by their serial } \\
\text { number's last digit, } \\
\text { West Jerusalem. } \\
\text { Questionnaire } \\
\text { (home) + } \\
\text { examination } \\
\text { (hospital). }\end{array}$} & \multirow[t]{2}{*}{277} & \multirow[t]{2}{*}{$?$} & \multirow[t]{2}{*}{$?$} & \multirow[t]{2}{*}{60} & \multirow[b]{2}{*}{$\begin{array}{l}\text { Subjects were asked } \\
\text { if they have back } \\
\text { pain. Further } \\
\text { questions on the } \\
\text { duration, frequency, } \\
\text { site, and severity of } \\
\text { their pain. Chronic } \\
\text { BP was defined as } \\
\text { reporting pain on } \\
\text { a frequent basis }\end{array}$} & \multirow[t]{2}{*}{ Self report } & \multirow[t]{2}{*}{ Point } & \multicolumn{3}{|l|}{70} & 44 & \multirow[t]{2}{*}{ U } \\
\hline & & & & & & & & & & 77 & & & 58 & \\
\hline \multirow[t]{2}{*}{ NP } & \multirow{2}{*}{$\begin{array}{l}\text { Andrianakos [41] } \\
2006 \text { Greece }\end{array}$} & \multirow[b]{2}{*}{$\begin{array}{l}\text { 1966-99, (19+ yo), } \\
\text { the total population } \\
\text { in } 7 \text { mixed } \\
\text { communities + } \\
\text { random sample in } \\
\text { another } 2 \text { mixed } \\
\text { communities. } \\
\text { Interview, } \\
\text { questionnaire + } \\
\text { examination } \\
\text { (home visit, } \\
\text { rheumatologist) }\end{array}$} & \multirow[t]{2}{*}{8740} & \multirow[t]{2}{*}{4269} & \multirow[t]{2}{*}{4471} & \multirow[t]{2}{*}{82} & \multirow{2}{*}{$\begin{array}{l}\text { NP localized in the } \\
\text { neck either radiating } \\
\text { or not along an } \\
\text { upper extremity }\end{array}$} & \multirow[t]{2}{*}{ Self report } & \multirow[t]{2}{*}{ Life time } & \multicolumn{3}{|l|}{ 59-64 } & 9 & $\mathrm{~L}$ \\
\hline & & & & & & & & & & \multicolumn{3}{|l|}{$69+$} & 8 & \\
\hline NP & $\begin{array}{l}\text { Chiu [61] } \\
2006 \\
\text { HongKong }\end{array}$ & $\begin{array}{l}2001,(15+\text { yo), } \\
\text { residents selected } \\
\text { through a two-stage } \\
\text { randomization } \\
\text { process. } \\
\text { Interview }+ \\
\text { questionnaire } \\
\text { (phone). }\end{array}$ & 664 & 295 & 364 & 66 & $\begin{array}{l}\text { "Up to the } \\
\text { present time, } \\
\text { have you ever had } \\
\text { neck pain?" + "at } \\
\text { least once in the } \\
\text { past } 12 \text { months" + } \\
\text { "within the past } \\
7 \text { days" }\end{array}$ & Self report & One year & \multicolumn{3}{|l|}{$65+$} & \multirow[t]{3}{*}{9.3} & U \\
\hline NP & $\begin{array}{l}\text { Hartvigsen [69] } \\
2006 \\
\text { Denmark }\end{array}$ & $\begin{array}{l}\text { 2003, (70-102 yo), } \\
\text { twins from the } \\
\text { populations-based } \\
\text { twin study (LSADT). } \\
\text { Interview + } \\
\text { questionnaire } \\
\text { (home). }\end{array}$ & 1844 & $?$ & $?$ & 84 & $\begin{array}{l}\text { Modified version of } \\
\text { the standardised } \\
\text { Nordic } \\
\text { Questionnaire } \\
\text { (SNQ) on } \\
\text { Musculoskeletal } \\
\text { Pain }\end{array}$ & Self report & One year & \multirow[t]{2}{*}{$72-102$} & $\begin{array}{l}16 \\
(13-19)\end{array}$ & \multirow[t]{2}{*}{$\begin{array}{l}20 \\
(18-22)\end{array}$} & & \multirow[t]{2}{*}{ U } \\
\hline NP & $\begin{array}{l}\text { Vogt [84] } \\
2003 \text { USA }\end{array}$ & $\begin{array}{l}\text { 1997-8, (70-79yo), a } \\
\text { random sample of } \\
\text { age-eligible white } \\
\text { Medicare } \\
\text { beneficiaries from } \\
\text { lists provided by } \\
\text { the Health Care } \\
\text { Financing } \\
\text { Administra-tion and } \\
\text { all age-eligible black }\end{array}$ & & & & & & & & & & & & \\
\hline
\end{tabular}


community residents

in designated zip

code areas close to

the Pittsburgh, PA,

and Memphis, TN,

field centers (the

Health $A B C$ study).

Interview +

examination

(home). 3075

1491

$\begin{array}{lll}\text { Neck or } & \text { Self } & \text { One year } \\ \text { shoulder } & \text { report } & \\ \text { pain lasting } & \\ \text { at least } & \\ 1 \text { month } & \\ \text { during the } & \\ \text { previous } & \\ \text { year } & \end{array}$

NP

Keenan [73]

1993, (55+ yo), a

two-stage random

sample from the

North Yorkshire

Services Authority.

Postal

questionnaire

$(65+$ yo), stratified

random sample

taken from the

population census

obtained from the

Catalan Statistics

Institute, Catalonia.

Interview +

questionnaire

(local primary

care centre)

$\begin{array}{ll}\text { Parsons [77] } & \text { 2001-3, (18+ yo), } \\ 2007 \text { UK } & \text { random samples } \\ & \text { from 16 Medical } \\ & \text { Research Council } \\ & \text { General Practice } \\ & \text { Research Framework } \\ & \text { practices, South } \\ & \text { East quadrant } \\ & \text { of the UK. } \\ & \text { Postal } \\ & \text { questionnaire }\end{array}$

86

Any swelling, pain, or stiffness in any of

lasted $>6$ weeks in

the previous 3

months. (identified on a manikin)

\begin{tabular}{|c|c|c|c|c|}
\hline 592 & 274 & 318 & 99 & $\begin{array}{l}\text { The Chronic Pain } \\
\text { Grade: "In the } \\
\text { past } 3 \text { months have } \\
\text { you had pain } \\
\text { that has lasted for } \\
\text { one day or longer } \\
\text { in any part of } \\
\text { your body?" }\end{array}$ \\
\hline 2501 & 1347 & 1154 & 47 & $\begin{array}{l}\text { The Chronic Pain } \\
\text { Grade: Any 'pain } \\
\text { which has lasted for } \\
3 \text { months or longer } \\
\text { and currently } \\
\text { troubles respondents } \\
\text { either all of the } \\
\text { time or on } \\
\text { and off' }\end{array}$ \\
\hline
\end{tabular}

70-79

11.9
$(10.8-13.0)$

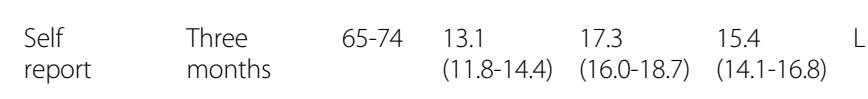

Self report Three months

$75+\quad 10.6$

$\begin{array}{lll}16.7 & 14.6\end{array}$

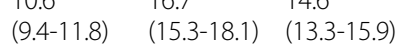

65-74

75-84

52.6

56.4

$85+$

53.5

Self report Three 
Table 7 Description of back pain (BP) and neck pain (NP) (Continued)

NP Strine [82] 2002, (18+ yo),

Multistage cluster

sample of random

all 50 states and

DC (the NHIS).

Interview +

questionnaire

(home).

Hartvigsen [68] 1995,1997,1999,

2004 Denmark 2001, (70-102 yo)

twins from the

populations-based

twin study (LSADT).

Interview +

questionnaire

(home).

$29828 ? ?$
$4484 \quad ? \quad ?$

"During the past 3

months did you have

Self report

Three

65

$+$

4.8

neck pain [lasting a

months

$(4.4-5.2)$

whole day or more

and not fleeting or

minor]?" [NB. NP

only, LBP not

included]

"Have you during the past month suffered

from pain or

stiffness in the

neck or shoulders?" +

diagnosis had

been made by

a physician

twins from the

populations-based

twin study (LSADT)

Interview +

questionnaire

(home).

Hartvigsen [70]

2005, (100 yo), all

2008 Denmark Danes born in 1905

were located

through the

Danish Civil

Registration

System.

Interview +

questionnaire

(home).

Thomas [87] (50+ yo), all patients

2004 UK

(50+ yo), all patients 7878 ? ?

from three GP

from the North

Staffordshire

Primary Care

Research

Consortium (the

NorStOP).

Postal

questionnaire

$1844 ?$ ?

$256 ?$ ?

"In the past 4 weeks

have you had pain

that has lasted for

one day or longer

in any part of

your body?"

[supplemented by

a full body
manikin]
Self

One month 60-69 
Table 7 Description of back pain (BP) and neck pain (NP) (Continued)

\begin{tabular}{|c|c|c|c|c|c|c|c|c|c|c|c|c|c|c|}
\hline \multirow[t]{2}{*}{ NP } & $\begin{array}{l}\text { Webb [85] } \\
2003 \text { UK }\end{array}$ & $\begin{array}{l}(16+\text { yo), stratified } \\
\text { sample of patients } \\
\text { from three GP in } \\
\text { West Pennine, } \\
\text { East of Manchester. } \\
\text { Questionnaire. }\end{array}$ & 4515 & $?$ & $?$ & 78 & $\begin{array}{l}\text { Pain lasting for more } \\
\text { than } 1 \text { week, over } \\
\text { the last month, in } \\
\text { any of seven areas } \\
\text { (back, neck, shoulder, } \\
\text { elbow, hand, hip, } \\
\text { knee) or in } \\
\text { multiple joints }\end{array}$ & $\begin{array}{l}\text { Self } \\
\text { report }\end{array}$ & $\begin{array}{l}\text { One } \\
\text { month }\end{array}$ & $65-74$ & 16.7 & 23.9 & & U \\
\hline & & & & & & & & & & $75+$ & 17.8 & 21.3 & & \\
\hline \multirow[t]{2}{*}{ NP } & $\begin{array}{l}\text { Natvig [76] } \\
2004 \text { Norway }\end{array}$ & $\begin{array}{l}\text { 1994, (24-76 yo), all } \\
\text { inhabitants in six } \\
\text { birth cohorts in } \\
\text { Ullensaker } \\
\text { municipality, } \\
\text { northeast of Oslo. } \\
\text { Postal } \\
\text { questionnaire }\end{array}$ & 3325 & 1501 & 1824 & 54 & $\begin{array}{l}\text { Standardised Nordic } \\
\text { Questionnaire: Any } \\
\text { pain or discomfort from } \\
\text { the neck during the } \\
\text { previous week } \\
\text { (illustrated on a } \\
\text { body mannequin) }\end{array}$ & $\begin{array}{l}\text { Self } \\
\text { report }\end{array}$ & $\begin{array}{l}\text { One } \\
\text { week }\end{array}$ & $64-66$ & & & 32.3 & U \\
\hline & & & & & & & & & & $\begin{array}{l}74-76 / \\
84-86\end{array}$ & & & 24.1 & \\
\hline NP & $\begin{array}{l}\text { Yaron [86] } \\
2011 \text { Israel }\end{array}$ & $\begin{array}{l}2002,2006,2008, \\
(20+\text { yo), stratified } \\
\text { sample drawn from } \\
\text { a telephone } \\
\text { database } \\
\text { on different } \\
\text { population sectors. } \\
\text { Telephone } \\
\text { interview + } \\
\text { questionnaire }\end{array}$ & 2520 & $47 \%$ & $53 \%$ & $59-66$ & $\begin{array}{l}\text { The Community } \\
\text { Oriented Program } \\
\text { for the Control of } \\
\text { Rheumatic Diseases } \\
\text { core questionnaire } \\
\text { (CCQ): "In the past } \\
7 \text { days have you } \\
\text { experienced pain } \\
\text { in any of the } \\
\text { following sites: } \\
\text { [ankles]?" }\end{array}$ & $\begin{array}{l}\text { Self } \\
\text { report }\end{array}$ & $\begin{array}{l}\text { One } \\
\text { week }\end{array}$ & $61+$ & & & 53.3 & U \\
\hline NP & $\begin{array}{l}\text { Goode [65] } \\
2010 \text { USA }\end{array}$ & $\begin{array}{l}\text { 2006, (21+ yo), } \\
\text { stratified random } \\
\text { probability sample } \\
\text { of North Carolina } \\
\text { telephone } \\
\text { numbers, } \\
\text { USA. } \\
\text { Interview + } \\
\text { questionnaire } \\
\text { (phone). }\end{array}$ & 2809 & $?$ & $?$ & 86 & $\begin{array}{l}\text { "Neck discomfort or } \\
\text { pain. Neck pain starts } \\
\text { in the neck area; it } \\
\text { may spread to the } \\
\text { shoulder or arm." } \\
\text { Chronic, impairing NP } \\
\text { 1) pain and activity } \\
\text { limitations nearly } \\
\text { every day for the } \\
\text { past } 3 \text { months or } \\
\text { 2) greater than } 24 \\
\text { episodes of pain in } \\
\text { the previous year, } \\
\text { with each episode } \\
\text { limiting activity for } \\
1 \text { day or more }\end{array}$ & $\begin{array}{l}\text { Self } \\
\text { report }\end{array}$ & Point & $65+$ & & & 1.2 & U \\
\hline NP & $\begin{array}{l}\text { Guez [67] } \\
2002 \text { Sweden }\end{array}$ & $\begin{array}{l}\text { 1999, }(25-74 \text { yo), } \\
\text { stratified randomised }\end{array}$ & 6000 & $?$ & $?$ & 72 & $\begin{array}{l}\text { "Have you visited a } \\
\text { doctor because of }\end{array}$ & Self report & Point & $65-74$ & 18 & 20 & & U \\
\hline
\end{tabular}


Table 7 Description of back pain (BP) and neck pain (NP) (Continued)

\begin{tabular}{|c|c|c|c|c|c|c|c|c|c|c|c|c|c|c|}
\hline & & $\begin{array}{l}\text { sample of inhabitants, } \\
\text { mainly along the } \\
\text { coastal area, } \\
\text { northern Sweden } \\
\text { (WHO MONICA } \\
\text { Study). } \\
\text { Questionnaire + } \\
\text { examination } \\
\text { (medical center) }\end{array}$ & & & & & $\begin{array}{l}\text { a neck or head } \\
\text { injury?", chronic NP } \\
\text { defined as } \\
\text { continuous } \\
\text { neck complaints for } \\
\text { more than } \\
6 \text { months }\end{array}$ & & & & & & & \\
\hline NP & $\begin{array}{l}\text { Picavet [78] } \\
2003 \text { The } \\
\text { Netherlands }\end{array}$ & $\begin{array}{l}\text { 1998, }(25+\text { yo), } \\
\text { stratified random } \\
\text { sample taken from } \\
\text { the population } \\
\text { register (the DMC3- } \\
\text { study). Postal } \\
\text { questionnaire }\end{array}$ & 3664 & $45 \%$ & $55 \%$ & 46 & $\begin{array}{l}\text { [Neck] pain during } \\
\text { the survey }\end{array}$ & $\begin{array}{l}\text { Self } \\
\text { report }\end{array}$ & Point & $65+$ & $\begin{array}{l}17.3 \\
(14.2-20.4)\end{array}$ & $\begin{array}{l}25.0 \\
(21.5-28.5)\end{array}$ & & L \\
\hline \multirow{3}{*}{$\begin{array}{l}\text { Thoracic } \\
\text { pain }\end{array}$} & \multirow{3}{*}{$\begin{array}{l}\text { Miro [75] } \\
2007 \text { Spain }\end{array}$} & \multirow{3}{*}{$\begin{array}{l}\text { ( } 65+\text { yo), stratified } \\
\text { random sample } \\
\text { taken from the } \\
\text { population census } \\
\text { obtained from the } \\
\text { Catalan Statistics } \\
\text { Institute, Catalonia. } \\
\text { Interview + } \\
\text { questionnaire } \\
\text { (local primary } \\
\text { care centre) }\end{array}$} & \multirow[t]{3}{*}{592} & \multirow[t]{3}{*}{274} & \multirow[t]{3}{*}{318} & \multirow[t]{3}{*}{99} & \multirow{3}{*}{$\begin{array}{l}\text { The Chronic Pain } \\
\text { Grade: "In the past } \\
3 \text { months have you } \\
\text { had pain that has } \\
\text { lasted for one day } \\
\text { or longer in any } \\
\text { part of your } \\
\text { body?" }\end{array}$} & \multirow{3}{*}{$\begin{array}{l}\text { Self } \\
\text { report }\end{array}$} & \multirow{3}{*}{$\begin{array}{l}\text { Three } \\
\text { months }\end{array}$} & $65-74$ & & & 15.0 & \multirow[t]{3}{*}{ L } \\
\hline & & & & & & & & & & $75-84$ & & & 12.9 & \\
\hline & & & & & & & & & & $85+$ & & & 11.6 & \\
\hline \multirow[t]{2}{*}{$\begin{array}{l}\text { Thoracic } \\
\text { pain }\end{array}$} & \multirow[t]{2}{*}{$\begin{array}{l}\text { Parsons [77] } \\
2007 \text { UK }\end{array}$} & \multirow[t]{2}{*}{$\begin{array}{l}\text { 2001-3, (18+ yo), } \\
\text { random samples } \\
\text { from } 16 \text { Medical } \\
\text { Research Council } \\
\text { General Practice } \\
\text { Research } \\
\text { Framework } \\
\text { practices, South } \\
\text { East quadrant } \\
\text { of the UK. } \\
\text { Postal } \\
\text { questionnaire }\end{array}$} & \multirow[t]{2}{*}{2501} & \multirow[t]{2}{*}{1347} & \multirow[t]{2}{*}{1154} & \multirow[t]{2}{*}{47} & \multirow[t]{2}{*}{$\begin{array}{l}\text { The Chronic Pain } \\
\text { Grade: Any 'pain } \\
\text { which has lasted for } \\
3 \text { months or longer } \\
\text { and currently } \\
\text { troubles } \\
\text { respondents } \\
\text { either all of the } \\
\text { time or on } \\
\text { and off' }\end{array}$} & \multirow[t]{2}{*}{$\begin{array}{l}\text { Self } \\
\text { report }\end{array}$} & \multirow[t]{2}{*}{$\begin{array}{l}\text { Three } \\
\text { months }\end{array}$} & $65-74$ & & & 2 & U \\
\hline & & & & & & & & & & 75-101 & & & 2 & \\
\hline $\begin{array}{l}\text { Higher } \\
\text { back }\end{array}$ & $\begin{array}{l}\text { Picavet [78] } \\
2003 \text { The } \\
\text { Netherlands }\end{array}$ & $\begin{array}{l}\text { 1998, (25+yo), } \\
\text { stratified random } \\
\text { sample taken } \\
\text { from the } \\
\text { population } \\
\text { register (the } \\
\text { DMC3-study). } \\
\text { Postal } \\
\text { questionnaire }\end{array}$ & 3664 & $45 \%$ & $55 \%$ & 46 & $\begin{array}{l}\text { [Higher part of } \\
\text { the back] pain } \\
\text { during the } \\
\text { survey }\end{array}$ & $\begin{array}{l}\text { Self } \\
\text { report }\end{array}$ & Point & $65+$ & $\begin{array}{l}2.8 \\
(1.4-4.2)\end{array}$ & $\begin{array}{l}11.9 \\
(9.2-14.6)\end{array}$ & & L \\
\hline
\end{tabular}


Overall, the one year prevalence ranged between 9\% and $12 \%[41,61,71,84]$. Greater variations were noted for the three-month prevalence, ranging between 5\% [77] and 56\% [75] in 65-74 year olds. Of the four one-month prevalence estimates using fairly similar NP definitions, about 23\% reported NP [70,76,85,87]. Men reported NP less often than women and in all studies there was a decrease in NP with increasing age, albeit small in some studies.

\section{Mid back pain}

Finally, MBP (i.e. thoracic or higher back pain) was reported in three studies $[75,77,78]$. The three-month prevalence was used in two studies, but with different MBP definitions and thus, the prevalence ranged between 2\% [77] and 15\% [75]. One study showed that pain in the "higher back" was four times more prevalent among women [78].

\section{Prevalence of shoulder pain}

Six studies reported five different prevalence periods on shoulder pain $[73,77,78,84,86,88]$ and two studies also included upper arm pain using two different prevalence periods $[87,89]$ (Table 8). Two studies (25\%) were rated as having low risk of bias $[73,77,78]$ and the rest as having an "unclear" risk of bias (Table 8 and Additional file 4).

All studies used different shoulder pain definition and/ or different prevalence periods. Nevertheless, in some of the studies with different prevalence periods, the estimates varied only slightly (3-5\%) (65-74 year olds, men: 10\%-13\%; women: $18 \%-23 \%)$ [73,78,89]. In three studies where gender estimates were provided, women reported more pain than men $[73,78,89]$. Only one study provided different age intervals, which showed that shoulder pain increased slightly with age.

\section{Prevalence of elbow pain}

Elbow pain was reported in four studies $[73,77,78,86]$ and elbow/forearm pain in one study [89], of which three different prevalence periods were used (Table 9). Two studies (40\%) were of low risk of bias [73,77,78], and the rest being unclear (Table 9 and Additional file 4).

Different elbow pain definitions were used in each study. Nevertheless, similar estimates were reported for both point and three-month prevalences [73,78]. Thus, approximately $5 \%$ of men and $6 \%-8 \%$ of women reported elbow pain. Elbow pain increased with age [73,77]. Fewer men reported elbow pain compared to women $[73,78]$.

\section{Prevalence of hand/wrist pain}

Two studies reported hand pain only $[73,87]$, one study wrist pain only [77], and three studies on combined wrist/hand pain $[78,86,89]$ (Table 10). Two studies (33\%) were of low risk of bias $[73,77,78]$, and the rest were unclear (Table 10 and Additional file 4).

Wrist and/or hand pain prevalence estimates varied greatly among the different studies. For example, as few as $14 \%$ of men aged $75+$ [73] and as many as $26 \%$ of women aged 60-69 [87] reported hand pain. Also, 2\% of men between 65-74 [89] and $22.5 \%$ of women (65+) [78] reported wrist/hand pain. Women reported more often wrist and/or hand pain than men $[73,78,89]$. Hand pain increased slightly with age in one study [73], but decreased in the other study [87].

\section{Prevalence of hip pain}

Five different prevalence periods on hip pain were reported in nine studies [73,75,77,78,83,87,90-92] (Table 11). Three studies (33\%) were considered to be of low risk of bias $[73,75,78]$ and only one study $(11 \%)$ of high risk of bias [83] (Table 11 and Additional file 4).

All nine studies used different hip pain definitions, resulting in a wide prevalence range. For example, the three-month prevalence ranged between $5 \%$ and $30 \%$ in the elderly aged 65-74 [73,75,77]. Six studies reported gender specific prevalence estimates, all of which reported a higher prevalence in women [73,78,83,90-92]. Age related changes were somewhat unclear and only showed small (2-4\%) differences across age groups.

\section{Prevalence of knee pain}

Eleven studies reported five different prevalence periods on knee pain $[27,73,77,78,83,86,87,91-94]$ (Table 12). Three studies $(27 \%)$ were of low risk of bias $[73,78,94]$ and one study being of high risk of bias [83] (Table 12 and Additional file 4).

All 11 studies used different pain definitions which resulted in great variations in prevalence estimates. For example, in the 65-74 year olds, the one-year prevalence varied between $26 \%$ and $70 \%$ in men and between $36 \%$ and $71 \%$ [91,92]. Generally, there was an increase in knee pain with increasing age, ranging between $3 \%$ and $8 \%[27,73,92,94]$. Some studies reported a slight decrease $[91,93]$ whereas others found no change with increasing age $[77,87]$. Five studies included gender specific prevalences and all showed that more women than men reported knee pain $[73,78,83,91,92]$.

\section{Prevalence of ankle/foot pain}

Nine studies included information on foot pain $[73,75,78,87,92,95-98]$, three studies on ankle pain $[78,86,99]$, and one study on both ankle/foot pain [77] (Table 13). Of these 12 studies in total, five (42\%) were of low risk of bias $[73,75,78,96,98]$ and only one study was considered being of high risk of bias [97] (Table 13 and Additional file 4). 
Table 8 Description of studies on shoulder pain

\begin{tabular}{|c|c|c|c|c|c|c|c|c|c|c|c|c|c|c|}
\hline & \multirow{2}{*}{$\begin{array}{l}\text { First author } \\
\text { Publ. year } \\
\text { Country }\end{array}$} & \multirow{2}{*}{$\begin{array}{l}\text { Study design / } \\
\text { Population /Method } \\
\text { of collection }\end{array}$} & \multicolumn{3}{|c|}{ Sample size } & \multirow{2}{*}{$\begin{array}{l}\text { Crude } \\
\text { response } \\
\text { rate }(\%)\end{array}$} & \multirow{2}{*}{$\begin{array}{l}\text { Outcome } \\
\text { definition }\end{array}$} & \multirow{2}{*}{$\begin{array}{l}\text { Outcome } \\
\text { assessment } \\
\text { method }\end{array}$} & \multirow{2}{*}{$\begin{array}{l}\text { Prevalence } \\
\text { period }\end{array}$} & \multirow[t]{2}{*}{ Age } & \multicolumn{3}{|c|}{ Prevalence* $(95 \% \mathrm{Cl})$} & \multirow{2}{*}{$\begin{array}{l}\text { Risk } \\
\text { of } \\
\text { bias }\end{array}$} \\
\hline & & & Total & $M$ & $F$ & & & & & & $M$ & $F$ & Total & \\
\hline \multirow{2}{*}{$\begin{array}{l}\text { Shoulder } \\
\text { pain }\end{array}$} & \multirow{2}{*}{$\begin{array}{l}\text { Hill [88] } \\
2010 \text { Australia }\end{array}$} & \multirow[b]{2}{*}{$\begin{array}{l}\text { 2004-6, (18+ yo), } \\
\text { recruited randomly } \\
\text { from the electronic } \\
\text { White Pages } \\
\text { telephone listings } \\
\text { (the NWAH Study). } \\
\text { Phone interview + } \\
\text { questionnaire }\end{array}$} & \multirow[t]{2}{*}{3488} & \multirow[t]{2}{*}{1712} & \multirow[t]{2}{*}{1776} & \multirow[t]{2}{*}{81} & \multirow{2}{*}{$\begin{array}{l}\text { Ever had pain or } \\
\text { aching in their } \\
\text { shoulder at rest } \\
\text { or when moving, } \\
\text { on most days for } \\
\text { at least a month }\end{array}$} & \multirow[t]{2}{*}{ Self report } & \multirow[t]{2}{*}{ Life time } & $65-74$ & & & 23.7 & \multirow[t]{2}{*}{ U } \\
\hline & & & & & & & & & & $75+$ & & & 26.5 & \\
\hline $\begin{array}{l}\text { Shoulder } \\
\text { pain }\end{array}$ & $\begin{array}{l}\text { Vogt [84] } \\
2003 \text { USA }\end{array}$ & $\begin{array}{l}\text { 1997-8, (70-79yo), a } \\
\text { random sample of } \\
\text { age-eligible white } \\
\text { Medicare beneficiaries } \\
\text { from lists provided } \\
\text { by the Health Care } \\
\text { Financing } \\
\text { Administration and } \\
\text { all age-eligible black } \\
\text { community residents } \\
\text { in designated zip } \\
\text { code areas close to } \\
\text { the Pittsburgh, PA, } \\
\text { and Memphis, TN, } \\
\text { field centers (the } \\
\text { Health ABC study). } \\
\text { Interview + } \\
\text { examination } \\
\text { (home) }\end{array}$ & 3075 & 1491 & 1584 & $?$ & $\begin{array}{l}\text { neck or shoulder } \\
\text { pain lasting at least } \\
1 \text { month during } \\
\text { the previous year }\end{array}$ & Self report & One year & $70-79$ & & & $\begin{array}{l}18.9 \\
(17.5-20.3)\end{array}$ & $U$ \\
\hline \multirow[t]{2}{*}{$\begin{array}{l}\text { Shoulder } \\
\text { pain }\end{array}$} & \multirow[t]{2}{*}{$\begin{array}{l}\text { Keenan [73] } \\
2006 \text { UK }\end{array}$} & \multirow{2}{*}{$\begin{array}{l}\text { 1993, (55+ yo), a } \\
\text { two-stage random } \\
\text { sample from the } \\
\text { North Yorkshire } \\
\text { Family Health } \\
\text { Services Authority. } \\
\text { Postal } \\
\text { questionnaire }\end{array}$} & \multirow[t]{2}{*}{16222} & \multirow[t]{2}{*}{$?$} & \multirow[t]{2}{*}{$?$} & \multirow[t]{2}{*}{86} & \multirow{2}{*}{$\begin{array}{l}\text { Any swelling, pain, } \\
\text { or stiffness in any } \\
\text { of their joints that } \\
\text { lasted }>6 \text { weeks } \\
\text { in the previous } \\
3 \text { months. } \\
\text { (identified on } \\
\text { a manikin) }\end{array}$} & \multirow[t]{2}{*}{ Self report } & \multirow[t]{2}{*}{ Three months } & $65-74$ & $\begin{array}{l}12.6 \\
(11.3-13.8)\end{array}$ & $\begin{array}{l}17.9 \\
(16.5-19.4)\end{array}$ & $\begin{array}{l}15.5 \\
(14.2-16.8)\end{array}$ & \multirow[t]{2}{*}{ L } \\
\hline & & & & & & & & & & $75+$ & $\begin{array}{l}13.1 \\
(11.2-14.3)\end{array}$ & $\begin{array}{l}21.0 \\
(19.5-22.4)\end{array}$ & $\begin{array}{l}18.3 \\
(16.8-19.7)\end{array}$ & \\
\hline \multirow{2}{*}{$\begin{array}{l}\text { Shoulder } \\
\text { pain }\end{array}$} & \multirow{2}{*}{$\begin{array}{l}\text { Parsons [77] } \\
2007 \text { UK }\end{array}$} & \multirow[b]{2}{*}{$\begin{array}{l}\text { 2001-3, (18+ yo), } \\
\text { random samples } \\
\text { from } 16 \text { Medical } \\
\text { Research Council } \\
\text { General Practice } \\
\text { Research } \\
\text { Framework } \\
\text { practices, South } \\
\text { East quadrant } \\
\text { of the UK. } \\
\text { Postal } \\
\text { questionnaire }\end{array}$} & \multirow[t]{2}{*}{2501} & \multirow[t]{2}{*}{1347} & 1154 & 47 & The Chronic Pain & Self report & Three months & $65-74$ & & & 4 & U \\
\hline & & & & & & & $\begin{array}{l}\text { Grade: Any 'pain } \\
\text { which has lasted for } \\
3 \text { months or longer } \\
\text { and currently } \\
\text { troubles respondents } \\
\text { either all of the } \\
\text { time or on } \\
\text { and off' }\end{array}$ & & & 75-101 & & & 3 & \\
\hline
\end{tabular}


Table 8 Description of studies on shoulder pain (Continued)

\begin{tabular}{|c|c|c|c|c|c|c|c|c|c|c|c|c|c|c|}
\hline $\begin{array}{l}\text { Shoulder } \\
\text { pain }\end{array}$ & $\begin{array}{l}\text { Yaron [86] } \\
2011 \text { Israel }\end{array}$ & $\begin{array}{l}\text { 2002, 2006, 2008, } \\
\text { (20+ yo), stratified } \\
\text { sample drawn } \\
\text { from a telephone } \\
\text { database on } \\
\text { different population } \\
\text { sectors. Telephone } \\
\text { Interview + } \\
\text { questionnaire }\end{array}$ & 2520 & $47 \%$ & $53 \%$ & $59-66$ & $\begin{array}{l}\text { The Community } \\
\text { Oriented Program } \\
\text { for the Control of } \\
\text { Rheumatic Diseases } \\
\text { core questionnaire } \\
\text { (CCQ): "In the past } \\
7 \text { days have you } \\
\text { experienced pain } \\
\text { in any of the } \\
\text { following sites: } \\
\text { [shoulders]?" }\end{array}$ & Self report & One week & $61+$ & & & 50.9 & U \\
\hline $\begin{array}{l}\text { Shoulder } \\
\text { pain }\end{array}$ & $\begin{array}{l}\text { Picavet [78] } \\
2003 \text { The } \\
\text { Netherlands }\end{array}$ & $\begin{array}{l}\text { 1998, (25+ yo), } \\
\text { stratified random } \\
\text { sample taken from } \\
\text { the population } \\
\text { register (the DMC3- } \\
\text { study). Postal } \\
\text { questionnaire }\end{array}$ & 3664 & $45 \%$ & $55 \%$ & 46 & $\begin{array}{l}\text { [Shoulder] pain } \\
\text { during the survey }\end{array}$ & Self report & Point & $65+$ & $\begin{array}{l}13.2 \\
(10.4-16.0)\end{array}$ & $\begin{array}{l}23.1 \\
(19.6-26.6)\end{array}$ & & L \\
\hline $\begin{array}{l}\text { Shoulder / } \\
\text { upper arm } \\
\text { pain }\end{array}$ & $\begin{array}{l}\text { Gummesson [89] } \\
2003 \text { Sweden }\end{array}$ & $\begin{array}{l}\text { 1997, (25-74 yo), } \\
\text { stratified randomised } \\
\text { sample from the } \\
\text { Swedish population } \\
\text { register in southern } \\
\text { Sweden. Postal } \\
\text { questionnaire }\end{array}$ & 2466 & $?$ & $?$ & 82 & $\begin{array}{l}\text { Chronic pain: } \\
\text { 'Where is the pain, } \\
\text { numbness, or } \\
\text { tingling located and } \\
\text { since when have } \\
\text { you had the } \\
\text { symptoms?' } \\
\text { [shoulder/upper } \\
\text { arm, since 3 } \\
\text { months] }\end{array}$ & Self report & Point & $65-74$ & 10.3 & 19.9 & & U \\
\hline \multirow{3}{*}{$\begin{array}{l}\text { Shoulder / } \\
\text { upper arm } \\
\text { pain }\end{array}$} & \multirow{3}{*}{$\begin{array}{l}\text { Thomas [87] } \\
2004 \text { UK }\end{array}$} & \multirow{3}{*}{$\begin{array}{l}(50+\text { yo), all patients } \\
\text { from three GPs from } \\
\text { the North } \\
\text { Staffordshire Primary } \\
\text { Care Research } \\
\text { Consortium (the } \\
\text { NorStOP). Postal } \\
\text { questionnaire }\end{array}$} & \multirow[t]{3}{*}{7878} & \multirow[t]{3}{*}{$?$} & \multirow[t]{3}{*}{$?$} & \multirow[t]{3}{*}{70} & \multirow{3}{*}{$\begin{array}{l}\text { "In the past } 4 \text { weeks } \\
\text { have you had pain } \\
\text { that has lasted } \\
\text { for one day or } \\
\text { longer in any part } \\
\text { of your body?" } \\
\text { [supplemented } \\
\text { by a full body } \\
\text { manikin] }\end{array}$} & \multirow[t]{3}{*}{ Self report } & \multirow[t]{3}{*}{ One month } & $60-69$ & & & 33.0 & \multirow[t]{3}{*}{ u } \\
\hline & & & & & & & & & & 70-79 & & & 28.0 & \\
\hline & & & & & & & & & & $80+$ & & & 24.9 & \\
\hline
\end{tabular}


Table 9 Description of studies on elbow pain

\begin{tabular}{|c|c|c|c|c|c|c|c|c|c|c|c|c|c|c|}
\hline & \multirow{2}{*}{$\begin{array}{l}\text { First author } \\
\text { Publ. year } \\
\text { Country }\end{array}$} & \multirow{2}{*}{$\begin{array}{l}\text { Study design / } \\
\text { Population /Method } \\
\text { of collection }\end{array}$} & \multicolumn{3}{|c|}{ Sample size } & \multirow{2}{*}{$\begin{array}{l}\text { Crude } \\
\text { response } \\
\text { rate }(\%)\end{array}$} & \multirow{2}{*}{$\begin{array}{l}\text { Outcome } \\
\text { definition }\end{array}$} & \multirow{2}{*}{$\begin{array}{l}\text { Outcome } \\
\text { assessment } \\
\text { method }\end{array}$} & \multirow{2}{*}{$\begin{array}{l}\text { Prevalence } \\
\text { period }\end{array}$} & \multirow[t]{2}{*}{ Age } & \multicolumn{3}{|c|}{ Prevalence* $(95 \% \mathrm{Cl})$} & \multirow{2}{*}{$\begin{array}{l}\text { Risk } \\
\text { of } \\
\text { bias }\end{array}$} \\
\hline & & & Total & $M$ & $F$ & & & & & & $M$ & $F$ & Total & \\
\hline \multirow{2}{*}{$\begin{array}{l}\text { Elbow } \\
\text { pain }\end{array}$} & \multirow{2}{*}{$\begin{array}{l}\text { Keenan [73] } \\
2006 \text { UK }\end{array}$} & \multirow[b]{2}{*}{$\begin{array}{l}\text { 1993, (55+ yo), a } \\
\text { two-stage random } \\
\text { sample from the } \\
\text { North Yorkshire } \\
\text { Family Health } \\
\text { Services Authority. } \\
\text { Postal } \\
\text { questionnaire }\end{array}$} & \multirow[t]{2}{*}{16222} & \multirow[t]{2}{*}{$?$} & \multirow[t]{2}{*}{$?$} & \multirow[t]{2}{*}{86} & \multirow{2}{*}{$\begin{array}{l}\text { any swelling, pain, } \\
\text { or stiffness in any } \\
\text { of their joints, that } \\
\text { lasted }>6 \text { weeks in } \\
\text { the previous } 3 \\
\text { months (identified } \\
\text { on a manikin) }\end{array}$} & \multirow[t]{2}{*}{ Self report } & \multirow{2}{*}{$\begin{array}{l}\text { Three } \\
\text { months }\end{array}$} & $65-74$ & $4.6(4.0-5.7)$ & $6.4(5.4-7.4)$ & $5.7(4.8-6.6)$ & $L$ \\
\hline & & & & & & & & & & $75+$ & $4.4(3.5-5.2)$ & $8.3(7.3-9.4)$ & $7.0(6.0-8.0)$ & \\
\hline \multirow{2}{*}{$\begin{array}{l}\text { Elbow } \\
\text { pain }\end{array}$} & \multirow{2}{*}{$\begin{array}{l}\text { Parsons [77] } \\
2007 \text { UK }\end{array}$} & \multirow[b]{2}{*}{$\begin{array}{l}\text { 2001-3, (18+ yo), } \\
\text { random samples } \\
\text { from } 16 \text { Medical } \\
\text { Research Council } \\
\text { General Practice } \\
\text { Research } \\
\text { Framework } \\
\text { practices, South } \\
\text { East quadrant } \\
\text { of the UK. } \\
\text { Postal } \\
\text { questionnaire }\end{array}$} & \multirow[t]{2}{*}{2501} & \multirow[t]{2}{*}{1347} & \multirow[t]{2}{*}{1154} & \multirow[t]{2}{*}{47} & \multirow[b]{2}{*}{$\begin{array}{l}\text { The Chronic Pain } \\
\text { Grade: Any 'pain } \\
\text { which has lasted } \\
\text { for } 3 \text { months or I } \\
\text { onger and currently } \\
\text { troubles respondents } \\
\text { either all of the } \\
\text { time or on } \\
\text { and off' }\end{array}$} & \multirow[t]{2}{*}{ Self report } & \multirow{2}{*}{$\begin{array}{l}\text { Three } \\
\text { months }\end{array}$} & $65-74$ & & 1 & $U$ & $U$ \\
\hline & & & & & & & & & & $75-101$ & & 2 & & \\
\hline $\begin{array}{l}\text { Elbow } \\
\text { pain }\end{array}$ & $\begin{array}{l}\text { Yaron [86] } \\
2011 \text { Israel }\end{array}$ & $\begin{array}{l}\text { 2002, 2006, 2008, } \\
\text { (20+ yo), stratified } \\
\text { sample drawn } \\
\text { from a telephone } \\
\text { database on } \\
\text { different population } \\
\text { sectors. Telephone } \\
\text { interview + } \\
\text { questionnaire }\end{array}$ & 2520 & $47 \%$ & $53 \%$ & $59-66$ & $\begin{array}{l}\text { The Community } \\
\text { Oriented Program } \\
\text { for the Control of } \\
\text { Rheumatic Diseases } \\
\text { core questionnaire } \\
\text { (CCQ): "In the past } 7 \\
\text { days have you } \\
\text { experienced pain } \\
\text { in any of the } \\
\text { following sites: } \\
\text { [elbow]?" }\end{array}$ & Self report & $\begin{array}{l}\text { One } \\
\text { week }\end{array}$ & $61+$ & & & 33.0 & $U$ \\
\hline $\begin{array}{l}\text { Elbow } \\
\text { pain }\end{array}$ & $\begin{array}{l}\text { Picavet [78] } \\
2003 \text { The } \\
\text { Netherlands }\end{array}$ & $\begin{array}{l}\text { 1998, ( } 25+\text { yo), } \\
\text { stratified random } \\
\text { sample taken from } \\
\text { the population } \\
\text { register (the DMC3- } \\
\text { study). Postal } \\
\text { questionnaire }\end{array}$ & 3664 & $45 \%$ & $55 \%$ & 46 & $\begin{array}{l}\text { [Elbow] pain during } \\
\text { the survey }\end{array}$ & Self report & Point & $65+$ & $4.9(3.1-6.7)$ & $8.0(5.8-10.2)$ & & L \\
\hline $\begin{array}{l}\text { Elbow/ } \\
\text { forearm } \\
\text { pain }\end{array}$ & $\begin{array}{l}\text { Gummesson [89] } \\
2003 \text { Sweden }\end{array}$ & $\begin{array}{l}\text { 1997, (25-74 yo), } \\
\text { stratified randomised } \\
\text { sample from the } \\
\text { Swedish population } \\
\text { register in southern } \\
\text { Sweden. Postal } \\
\text { questionnaire }\end{array}$ & 2466 & $?$ & $?$ & 82 & $\begin{array}{l}\text { Chronic pain: 'Where } \\
\text { is the pain, numbness, } \\
\text { or tingling located } \\
\text { and since when have } \\
\text { you had the symptoms?' } \\
\text { [elbow/forearm, } \\
\text { since } 3 \text { months] }\end{array}$ & Self report & Point & $65-74$ & 1.7 & 8.3 & & $U$ \\
\hline
\end{tabular}

*Prevalence estimates without decimals are obtained from figures/graphs in the article and should be interpreted with caution.

I: Interview, Q: Questionnaire; E: Examination, R: Register. L: Low, U: Unclear, H: High.

GP: General practitioner; ACR: The American College of Rheumatology (ACR clinical criteria for RA [22]). 
Table 10 Description of studies on wrist and hand pain

\begin{tabular}{|c|c|c|c|c|c|c|c|c|c|c|c|c|c|c|}
\hline & \multirow{2}{*}{$\begin{array}{l}\text { First author } \\
\text { Publ. year } \\
\text { Country }\end{array}$} & \multirow{2}{*}{$\begin{array}{l}\text { Study design / } \\
\text { Population / Method } \\
\text { of collection }\end{array}$} & \multicolumn{3}{|c|}{ Sample size } & \multirow{2}{*}{$\begin{array}{l}\text { Crude } \\
\text { response } \\
\text { rate (\%) }\end{array}$} & \multirow{2}{*}{$\begin{array}{l}\text { Outcome } \\
\text { definition }\end{array}$} & \multirow{2}{*}{$\begin{array}{l}\text { Outcome } \\
\text { assessment } \\
\text { method }\end{array}$} & \multirow{2}{*}{$\begin{array}{l}\text { Prevalence } \\
\text { period }\end{array}$} & \multirow[t]{2}{*}{ Age } & \multicolumn{3}{|c|}{ Prevalence* $(95 \% \mathrm{Cl})$} & \multirow{2}{*}{$\begin{array}{l}\text { Risk } \\
\text { of } \\
\text { bias }\end{array}$} \\
\hline & & & Total & M & $\mathrm{F}$ & & & & & & M & $\mathrm{F}$ & Total & \\
\hline \multirow{2}{*}{$\begin{array}{l}\text { Hand } \\
\text { pain }\end{array}$} & \multirow{2}{*}{$\begin{array}{l}\text { Keenan [73] } \\
2006 \text { UK }\end{array}$} & \multirow[b]{2}{*}{$\begin{array}{l}\text { 1993, (55+ yo), a } \\
\text { two-stage random } \\
\text { sample from the } \\
\text { North Yorkshire } \\
\text { Family Health } \\
\text { Services Authority. } \\
\text { Postal } \\
\text { questionnaire }\end{array}$} & \multirow[t]{2}{*}{16222} & \multirow[t]{2}{*}{$?$} & \multirow[t]{2}{*}{$?$} & \multirow[t]{2}{*}{86} & \multirow{2}{*}{$\begin{array}{l}\text { Any swelling, pain, } \\
\text { or stiffness in any } \\
\text { of their joints that } \\
\text { lasted }>6 \text { weeks in } \\
\text { the previous } 3 \\
\text { months. (identified } \\
\text { on a manikin) }\end{array}$} & \multirow[t]{2}{*}{ Self report } & \multirow[t]{2}{*}{3 months } & $65-74$ & $14.2(13.0-15.6)$ & $23.3(21.8-24.9)$ & $19.2(17.8-20.6)$ & L \\
\hline & & & & & & & & & & $75+$ & $11.6(10.4-12.8)$ & $25.3(23.7-26.8)$ & $20.6(19.1-22.1)$ & \\
\hline \multirow{3}{*}{$\begin{array}{l}\text { Hand } \\
\text { pain }\end{array}$} & \multirow{3}{*}{$\begin{array}{l}\text { Thomas [87] } \\
2004 \text { UK }\end{array}$} & \multirow{3}{*}{$\begin{array}{l}\text { ( } 50+\text { yo), all patients } \\
\text { from three GPs } \\
\text { from the North } \\
\text { Staffordshire } \\
\text { Primary Care } \\
\text { Research Consortium } \\
\text { (the NorStOP). } \\
\text { Postal } \\
\text { questionnaire }\end{array}$} & \multirow[t]{3}{*}{7878} & \multirow[t]{3}{*}{$?$} & \multirow[t]{3}{*}{$?$} & \multirow[t]{3}{*}{70} & \multirow{3}{*}{$\begin{array}{l}\text { "In the past } 4 \text { weeks } \\
\text { have you had pain } \\
\text { that has lasted for } \\
\text { one day or longer } \\
\text { in any part of your } \\
\text { body?" [supplemented } \\
\text { by a full body } \\
\text { manikin] }\end{array}$} & \multirow[t]{3}{*}{ Self report } & \multirow[t]{3}{*}{ One month } & $60-69$ & & & 25.6 & U \\
\hline & & & & & & & & & & $70-79$ & & & 20.2 & \\
\hline & & & & & & & & & & $80+$ & & & 16.9 & \\
\hline \multirow{2}{*}{$\begin{array}{l}\text { Wrist } \\
\text { pain }\end{array}$} & \multirow{2}{*}{$\begin{array}{l}\text { Parsons [77] } \\
2007 \text { UK }\end{array}$} & \multirow[b]{2}{*}{$\begin{array}{l}\text { 2001-3, (18+ yo), } \\
\text { random samples } \\
\text { from } 16 \text { Medical } \\
\text { Research Council } \\
\text { General Practice } \\
\text { Research } \\
\text { Framework } \\
\text { practices, South } \\
\text { East quadrant of } \\
\text { the UK. Postal } \\
\text { questionnaire }\end{array}$} & \multirow[t]{2}{*}{2501} & \multirow[t]{2}{*}{1347} & \multirow[t]{2}{*}{1154} & 47 & The Chronic Pain & Self report & 3 months & $65-74$ & & & 4 & $U$ \\
\hline & & & & & & & $\begin{array}{l}\text { Grade: Any pain } \\
\text { which has lasted } \\
\text { for } 3 \text { months or } \\
\text { longer and currently } \\
\text { troubles respondents } \\
\text { either all of the } \\
\text { time or on } \\
\text { and off' }\end{array}$ & & & 75-101 & & & 3 & \\
\hline $\begin{array}{l}\text { Wrist/ } \\
\text { hand } \\
\text { pain }\end{array}$ & $\begin{array}{l}\text { Yaron [86] } \\
2011 \text { Israel }\end{array}$ & $\begin{array}{l}\text { 2002, 2006, 2008, } \\
\text { (20+ yo), stratified } \\
\text { sample drawn from } \\
\text { a telephone } \\
\text { database on } \\
\text { different population } \\
\text { sectors. Telephone } \\
\text { interview + } \\
\text { questionnaire }\end{array}$ & 2520 & $47 \%$ & $53 \%$ & $59-66$ & $\begin{array}{l}\text { The Community } \\
\text { Oriented Program } \\
\text { for the Control of } \\
\text { Rheumatic Diseases } \\
\text { core questionnaire } \\
\text { (CCQ): "In the past } \\
7 \text { days have you } \\
\text { experienced pain } \\
\text { in any of the } \\
\text { following sites: } \\
\text { [hands/wrists]?" }\end{array}$ & Self report & One week & $61+$ & & & 33.0 & $U$ \\
\hline $\begin{array}{l}\text { Wrist/ } \\
\text { hand } \\
\text { pain }\end{array}$ & $\begin{array}{l}\text { Gummesson [89] } \\
2003 \text { Sweden }\end{array}$ & $\begin{array}{l}\text { 1997, (25-74 yo), } \\
\text { stratified randomised } \\
\text { sample from the } \\
\text { Swedish population } \\
\text { register in southern } \\
\text { Sweden. Postal } \\
\text { questionnaire }\end{array}$ & 2466 & $?$ & $?$ & 82 & $\begin{array}{l}\text { Chronic pain: 'Where is } \\
\text { the pain, numbness, } \\
\text { or tingling located } \\
\text { and since when have } \\
\text { you had the symptoms?' } \\
\text { [wrist/hand, since } \\
3 \text { months] }\end{array}$ & Self report & Point & $65-74$ & 2.1 & 14.9 & & U \\
\hline
\end{tabular}


Table 10 Description of studies on wrist and hand pain (Continued)

\begin{tabular}{|c|c|c|c|c|c|c|c|c|c|c|c|c|c|}
\hline $\begin{array}{l}\text { Wrist/ } \\
\text { hand } \\
\text { pain }\end{array}$ & $\begin{array}{l}\text { Picavet [78] } \\
2003 \text { The } \\
\text { Netherlands }\end{array}$ & $\begin{array}{l}\text { 1998, }(25+\text { yo), } \\
\text { stratified random } \\
\text { sample taken from } \\
\text { the population } \\
\text { register (the DMC3- } \\
\text { study). Postal } \\
\text { questionnaire }\end{array}$ & 3664 & $45 \%$ & $55 \%$ & 46 & $\begin{array}{l}\text { [Wrist/hand] pain } \\
\text { during the survey }\end{array}$ & Self report & Point & $65+$ & $9.7(7.3-12.1)$ & $22.5(19.1-25.9)$ & $\mathrm{L}$ \\
\hline
\end{tabular}

*Prevalence estimates without decimals are obtained from figures/graphs in the article and should be interpreted with caution

L: Low, U: Unclear, H: High.

GP: General practitioner; ACR: The American College of Rheumatology (ACR clinical criteria for RA [22]). 
Table 11 Description of studies on hip pain

\begin{tabular}{|c|c|c|c|c|c|c|c|c|c|c|c|c|c|}
\hline \multirow{2}{*}{$\begin{array}{l}\text { First author } \\
\text { Publ. year } \\
\text { Country }\end{array}$} & \multirow{2}{*}{$\begin{array}{l}\text { Study design / } \\
\text { Population /Method } \\
\text { of collection }\end{array}$} & \multicolumn{3}{|c|}{ Sample size } & \multirow{2}{*}{$\begin{array}{l}\text { Crude } \\
\text { response } \\
\text { rate }(\%)\end{array}$} & \multirow{2}{*}{$\begin{array}{l}\text { Outcome } \\
\text { definition }\end{array}$} & \multirow{2}{*}{$\begin{array}{l}\text { Outcome } \\
\text { assessment } \\
\text { method }\end{array}$} & \multirow{2}{*}{$\begin{array}{l}\text { Prevalence } \\
\text { period }\end{array}$} & \multirow[t]{2}{*}{ Age } & \multicolumn{3}{|c|}{ Prevalence* $(95 \% \mathrm{Cl})$} & \multirow{2}{*}{$\begin{array}{l}\text { Risk } \\
\text { of } \\
\text { bias }\end{array}$} \\
\hline & & Total & $M$ & $\mathrm{~F}$ & & & & & & $M$ & $\mathrm{~F}$ & Total & \\
\hline \multirow{2}{*}{$\begin{array}{l}\text { Peat [92] } \\
2006 \text { UK }\end{array}$} & \multirow[b]{2}{*}{$\begin{array}{l}2002,(50+\text { yo), all } \\
\text { community-dwelling } \\
\text { adults registered with } \\
3 \text { general practices in } \\
\text { North Staffordshire } \\
\text { (The NorStOP). } \\
\text { Postal } \\
\text { questionnaire }\end{array}$} & \multirow[t]{2}{*}{2429} & \multirow[t]{2}{*}{1005} & \multirow[t]{2}{*}{1424} & \multirow[t]{2}{*}{22} & \multirow[b]{2}{*}{$\begin{array}{l}\text { The Regional Pains } \\
\text { Survey, containing } \\
\text { the Western } \\
\text { Ontario } \\
\& \text { McMaster } \\
\text { Universities } \\
\text { Osteoarthritis Index } \\
\text { on hip pain } \\
\text { (the WOMAC-HIP) }\end{array}$} & \multirow[t]{2}{*}{ Self report } & \multirow[t]{2}{*}{ One year } & $65-74$ & 47 & 50 & & $U$ \\
\hline & & & & & & & & & $75+$ & 44 & 48 & & \\
\hline \multirow{3}{*}{$\begin{array}{l}\text { Dawson [91] } \\
2004 \text { UK }\end{array}$} & \multirow{3}{*}{$\begin{array}{l}2002,(65+) \text {, a random } \\
\text { sample from the } \\
\text { Oxfordshire Health } \\
\text { Authority register. } \\
\text { Postal } \\
\text { questionnaire }\end{array}$} & \multirow[t]{3}{*}{3341} & \multirow[t]{3}{*}{1557} & \multirow[t]{3}{*}{1784} & \multirow[t]{3}{*}{61} & \multirow{3}{*}{$\begin{array}{l}\text { "During the past } \\
12 \text { months, have } \\
\text { you had pain in } \\
\text { or around either } \\
\text { of your hips on } \\
\text { most days for } \\
\text { one month or } \\
\text { longer?" }\end{array}$} & \multirow[t]{3}{*}{ Self report } & \multirow[t]{3}{*}{ One year } & $65-74$ & 14.7 & 23.1 & & U \\
\hline & & & & & & & & & $75-84$ & 18.0 & 20.7 & & \\
\hline & & & & & & & & & $85+$ & 18.8 & 21.0 & & \\
\hline \multirow{2}{*}{$\begin{array}{l}\text { Keenan [73] } \\
2006 \text { UK }\end{array}$} & \multirow[b]{2}{*}{$\begin{array}{l}\text { 1993, (55+ yo), a } \\
\text { two-stage random } \\
\text { sample from the } \\
\text { North Yorkshire } \\
\text { Family Health } \\
\text { Services Authority. } \\
\text { Postal } \\
\text { questionnaire }\end{array}$} & \multirow[t]{2}{*}{16222} & \multirow[t]{2}{*}{$?$} & \multirow[t]{2}{*}{$?$} & \multirow[t]{2}{*}{86} & \multirow[b]{2}{*}{$\begin{array}{l}\text { Any swelling, pain, } \\
\text { or stiffness in any } \\
\text { of their joints that } \\
\text { lasted }>6 \text { weeks } \\
\text { in the previous } \\
3 \text { months } \\
\text { (identified on } \\
\text { a manikin) }\end{array}$} & Self report & 3 months & $65-74$ & $10.2(9.1-11.4)$ & $14.4(13.1-15.8)$ & $12.5(12.3-13.8)$ & $L$ \\
\hline & & & & & & & & & $75+$ & $7.3(6.3-8.4)$ & $17.2(15.8-18.6)$ & $13.8(12.6-15.1)$ & \\
\hline Miro [75] & $(65+$ yo), stratified & 592 & 274 & 318 & 99 & The Chronic Pain & Self report & 3 months & $65-74$ & & & 30.3 & L \\
\hline & $\begin{array}{l}\text { random sample taken } \\
\text { from the population }\end{array}$ & & & & & $\begin{array}{l}\text { Grade: In the past } \\
3 \text { months have }\end{array}$ & & & $75-84$ & & & 31.5 & \\
\hline & $\begin{array}{l}\text { census obtained from } \\
\text { the Catalan Statistics } \\
\text { Institute, Catalonia. } \\
\text { Interview + } \\
\text { questionnaire } \\
\text { (local primary } \\
\text { care centre) }\end{array}$ & & & & & $\begin{array}{l}\text { you had pain that } \\
\text { has lasted for one } \\
\text { day or longer in } \\
\text { any part of your } \\
\text { body?" }\end{array}$ & & & $85+$ & & & 30.2 & \\
\hline Parsons [77] & 2001-3, (18+ yo), & 2501 & 1347 & 1154 & 47 & The Chronic Pain & Self report & 3 months & $65-74$ & & & 5 & $U$ \\
\hline & $\begin{array}{l}\text { from } 16 \text { Medical } \\
\text { Research Council } \\
\text { General Practice } \\
\text { Research } \\
\text { Framework } \\
\text { practices, South } \\
\text { East quadrant of } \\
\text { the UK. Postal }\end{array}$ & & & & & $\begin{array}{l}\text { which has lasted } \\
\text { for } 3 \text { months or } \\
\text { longer and } \\
\text { currently troubles } \\
\text { respondents } \\
\text { either all of the } \\
\text { time or on } \\
\text { and off' }\end{array}$ & & & 75-101 & & & 4 & \\
\hline
\end{tabular}


Table 11 Description of studies on hip pain (Continued)

\begin{tabular}{|c|c|c|c|c|c|c|c|c|c|c|c|c|c|}
\hline \multirow{3}{*}{$\begin{array}{l}\text { Christmas [90] } \\
2002 \text { USA }\end{array}$} & \multirow[b]{3}{*}{$\begin{array}{l}1988-92 \text { \& 1991-4, } \\
(60+\text { yo), a } \\
\text { multistage, } \\
\text { cluster and } \\
\text { stratified } \\
\text { representative } \\
\text { sample of US } \\
\text { civilians } \\
\text { (NHANES III). Home } \\
\text { Questionnaire } \\
\text { and Interview, } \\
\text { Examination in } \\
\text { mobile } \\
\text { examination } \\
\text { centre }\end{array}$} & \multirow[t]{3}{*}{6596} & \multirow[t]{3}{*}{$?$} & \multirow[t]{3}{*}{$?$} & \multirow[t]{3}{*}{ ? } & \multirow{3}{*}{$\begin{array}{l}\text { Significant hip } \\
\text { pain on most } \\
\text { days over the } \\
\text { preceding } \\
6 \text { weeks }\end{array}$} & \multirow{3}{*}{$\begin{array}{l}\text { Self report } \\
\& \text { clinical } \\
\text { examination }\end{array}$} & \multirow[t]{3}{*}{6 week } & $60-69$ & 11 & \multicolumn{2}{|l|}{14} & \multirow[t]{3}{*}{ U } \\
\hline & & & & & & & & & 70-79 & 12 & 17 & & \\
\hline & & & & & & & & & $80+$ & 11 & 16 & & \\
\hline $\begin{array}{l}\text { Suka [83] } \\
2009 \text { Japan }\end{array}$ & $\begin{array}{l}\text { 2005, about } 1000 \\
\text { persons from five } \\
\text { different healthcare } \\
\text { facilities were asked } \\
\text { to participate. } \\
\text { Questionnaire } \\
\text { (Health care } \\
\text { facility) }\end{array}$ & 5652 & $?$ & $?$ & $?$ & $\begin{array}{l}\text { Musculoskeletal } \\
\text { pain (marked on } \\
\text { a drawing with } \\
\text { predefined body } \\
\text { regions) for more } \\
\text { than } 1 \text { week } \\
\text { during the } \\
\text { last month }\end{array}$ & Self report & One month & $60-69$ & 2.4 & 5.6 & & $\mathrm{H}$ \\
\hline \multirow{3}{*}{$\begin{array}{l}\text { Thomas [87] } \\
2004 \text { UK }\end{array}$} & \multirow{3}{*}{$\begin{array}{l}\text { (50+ yo), all patients } \\
\text { from three GPs from } \\
\text { the North } \\
\text { Staffordshire } \\
\text { Primary Care } \\
\text { Research } \\
\text { Consortium (the } \\
\text { NorStOP). Postal } \\
\text { questionnaire }\end{array}$} & \multirow[t]{3}{*}{7878} & \multirow[t]{3}{*}{$?$} & \multirow[t]{3}{*}{$?$} & \multirow[t]{3}{*}{70} & \multirow{3}{*}{$\begin{array}{l}\text { "In the past } 4 \text { weeks } \\
\text { have you had pain } \\
\text { that has lasted for } \\
\text { one day or longer } \\
\text { in any part } \\
\text { of your body?" } \\
\text { [supplemented } \\
\text { by a full body } \\
\text { manikin] }\end{array}$} & \multirow[t]{3}{*}{ Self report } & \multirow[t]{3}{*}{ One month } & $60-69$ & & & 28.3 & \multirow[t]{3}{*}{ U } \\
\hline & & & & & & & & & 70-79 & & & 27.0 & \\
\hline & & & & & & & & & $80+$ & & & 25.6 & \\
\hline $\begin{array}{l}\text { Picavet [78] } \\
2003 \text { The } \\
\text { Netherlands }\end{array}$ & $\begin{array}{l}\text { 1998, ( } 25+\text { yo), } \\
\text { stratified random } \\
\text { sample taken } \\
\text { from the population } \\
\text { register (the DMC3- } \\
\text { study). Postal } \\
\text { questionnaire }\end{array}$ & 3664 & $44.8 \%$ & $55.2 \%$ & 46 & $\begin{array}{l}\text { [Hip] pain during } \\
\text { the survey }\end{array}$ & Self report & Point & $65+$ & $11.1(8.5-13.7)$ & $21.2(17.8-24.5)$ & & L \\
\hline
\end{tabular}


Table 12 Description of studies on knee pain

\begin{tabular}{|c|c|c|c|c|c|c|c|c|c|c|c|c|c|}
\hline \multirow{2}{*}{$\begin{array}{l}\text { First author } \\
\text { Publ. year } \\
\text { Country }\end{array}$} & \multirow{2}{*}{$\begin{array}{l}\text { Study design / } \\
\text { Population / Method } \\
\text { of collection }\end{array}$} & \multicolumn{3}{|c|}{ Sample size } & \multirow{2}{*}{$\begin{array}{l}\text { Crude } \\
\text { response } \\
\text { rate }(\%)\end{array}$} & \multirow{2}{*}{$\begin{array}{l}\text { Outcome } \\
\text { definition }\end{array}$} & \multirow{2}{*}{$\begin{array}{l}\text { Outcome } \\
\text { assessment } \\
\text { method }\end{array}$} & \multirow{2}{*}{$\begin{array}{l}\text { Prevalence } \\
\text { period }\end{array}$} & \multirow[t]{2}{*}{ Age } & \multicolumn{3}{|c|}{ Prevalence* $(95 \% \mathrm{Cl})$} & \multirow{2}{*}{$\begin{array}{l}\text { Risk } \\
\text { of } \\
\text { bias }\end{array}$} \\
\hline & & Total & $M$ & $F$ & & & & & & $M$ & $\mathrm{~F}$ & Total & \\
\hline \multirow{3}{*}{$\begin{array}{l}\text { Dawson [91] } \\
2004 \text { UK }\end{array}$} & \multirow{3}{*}{$\begin{array}{l}2002,(65+), \text { a } \\
\text { random sample } \\
\text { from the } \\
\text { Oxfordshire } \\
\text { Health Authority } \\
\text { register. Postal } \\
\text { questionnaire }\end{array}$} & \multirow[t]{3}{*}{3341} & \multirow[t]{3}{*}{1557} & \multirow[t]{3}{*}{1784} & \multirow[t]{3}{*}{61} & \multirow{3}{*}{$\begin{array}{l}\text { "During the past } 12 \\
\text { months, have you } \\
\text { had pain in or around } \\
\text { either of your hips } \\
\text { on most days for } \\
\text { one month or } \\
\text { longer?" }\end{array}$} & \multirow[t]{3}{*}{ Self report } & \multirow[t]{3}{*}{ One year } & $65-74$ & 26.1 & 36.2 & & $U$ \\
\hline & & & & & & & & & $75-84$ & 31.0 & 37.4 & & \\
\hline & & & & & & & & & $85+$ & 32.3 & 35.5 & & \\
\hline \multirow{2}{*}{$\begin{array}{l}\text { Jinks [94] } \\
2008 \text { UK }\end{array}$} & \multirow{2}{*}{$\begin{array}{l}(50+) \text {, all pxatients } \\
\text { registered at } \\
\text { three general } \\
\text { practices in North } \\
\text { Staffordshire. } \\
\text { Postal } \\
\text { questionnaire }\end{array}$} & \multirow[t]{2}{*}{2059} & \multirow[t]{2}{*}{$?$} & \multirow[t]{2}{*}{$?$} & \multirow[t]{2}{*}{56} & \multirow{2}{*}{$\begin{array}{l}\text { Have had pain in or } \\
\text { around either knee } \\
\text { in the last } 12 \text { months } \\
\text { (NB. Only 'severe' } \\
\text { pain can be extracted } \\
\text { from "new onset" } \\
\text { of knee pain) }\end{array}$} & \multirow[t]{2}{*}{ Self report } & \multirow[t]{2}{*}{ One year } & $65-74$ & & & 8 & L \\
\hline & & & & & & & & & $75+$ & & & 12 & \\
\hline \multirow{2}{*}{$\begin{array}{l}\text { Peat [92] } \\
2006 \text { UK }\end{array}$} & \multirow[b]{2}{*}{$\begin{array}{l}\text { 2002, (50+ yo), } \\
\text { all community- } \\
\text { dwelling adults } \\
\text { registered with } 3 \\
\text { general practices } \\
\text { in North Staffordshire } \\
\text { (The NorStOP). } \\
\text { Postal } \\
\text { questionnaire }\end{array}$} & \multirow[t]{2}{*}{2429} & \multirow[t]{2}{*}{1005} & \multirow[t]{2}{*}{1424} & \multirow[t]{2}{*}{22} & \multirow[b]{2}{*}{$\begin{array}{l}\text { The Regional Pains } \\
\text { Survey, containing } \\
\text { the Western } \\
\text { Ontario \& } \\
\text { McMaster } \\
\text { Universities } \\
\text { Osteoarthritis Index } \\
\text { on hip pain (the } \\
\text { WOMAC-KNEE) }\end{array}$} & \multirow[t]{2}{*}{ Self report } & One year & $65-74$ & 70 & 71 & & $U$ \\
\hline & & & & & & & & & $75+$ & 62 & 74 & & \\
\hline Keenan [73] & 1993, (55+ yo), a & 16222 & $?$ & $?$ & $q$ & Any swelling, pain, & Self & Three & $65-74$ & $18.7(17.3-?)$ & $24.2(22.6-25.7)$ & $21.7(20.2-23.2)$ & $\mathrm{L}$ \\
\hline & $\begin{array}{l}\text { two-stage random } \\
\text { sample from the } \\
\text { North Yorkshire } \\
\text { Family Health } \\
\text { Services Authority. } \\
\text { Postal } \\
\text { questionnaire }\end{array}$ & & & & & $\begin{array}{l}\text { or stiffness in any of } \\
\text { their joints that lasted } \\
>6 \text { weeks in the } \\
\text { previous } 3 \text { months. } \\
\text { (identified on a } \\
\text { manikin) }\end{array}$ & & months & $75+$ & $17.4(16.0-18.8)$ & $31.2(29.5-32.8)$ & $26.4(24.9-28.0)$ & \\
\hline Parsons [77] & 2001-3, (18+yo), & 2501 & 1347 & 1154 & 47 & The Chronic Pain & Self & Three & $65-74$ & & & 6 & $U$ \\
\hline & $\begin{array}{l}\text { random samples } \\
\text { from } 16 \text { Medical } \\
\text { Research Council } \\
\text { General Practice } \\
\text { Research } \\
\text { Framework } \\
\text { practices, South } \\
\text { East quadrant of } \\
\text { the UK. Postal } \\
\text { questionnaire }\end{array}$ & & & & & $\begin{array}{l}\text { Grade: Any 'pain } \\
\text { which has lasted for } 3 \\
\text { months or longer and } \\
\text { currently troubles } \\
\text { respondents either } \\
\text { all of the time or on } \\
\text { and off' }\end{array}$ & & months & $75-101$ & & & 6 & \\
\hline
\end{tabular}


Table 12 Description of studies on knee pain (Continued)

\begin{tabular}{|c|c|c|c|c|c|c|c|c|c|c|c|c|c|}
\hline \multirow{2}{*}{$\begin{array}{l}\text { Croft [93] } \\
2005 \text { UK }\end{array}$} & \multirow{2}{*}{$\begin{array}{l}(50+) \text {, all patients } \\
\text { registered at three } \\
\text { general practices } \\
\text { in North } \\
\text { Staffordshire }\end{array}$} & \multirow[t]{2}{*}{5346} & \multirow[t]{2}{*}{$45 \%$} & \multirow[t]{2}{*}{$55 \%$} & \multirow[t]{2}{*}{59} & \multirow{2}{*}{$\begin{array}{l}\text { 'Draw on a blank } \\
\text { body manikin any } \\
\text { pain or ache that had } \\
\text { lasted for } \geq 1 \text { day } \\
\text { in the last } \\
\text { month' }\end{array}$} & \multirow{2}{*}{$\begin{array}{l}\text { Self } \\
\text { report }\end{array}$} & \multirow{2}{*}{$\begin{array}{l}\text { One } \\
\text { month }\end{array}$} & \multicolumn{3}{|l|}{$65-74$} & 63.4 & \multirow[t]{2}{*}{ U } \\
\hline & & & & & & & & & $75+$ & & & 60.4 & \\
\hline $\begin{array}{l}\text { Suka [83] } \\
2009 \text { Japan }\end{array}$ & $\begin{array}{l}\text { 2005, about } 1000 \\
\text { persons from } \\
\text { five different } \\
\text { healthcare facilities } \\
\text { were asked to } \\
\text { participate. } \\
\text { Interview }+ \\
\text { questionnaire } \\
\text { (Health care } \\
\text { facility) }\end{array}$ & 5652 & $?$ & $?$ & $?$ & $\begin{array}{l}\text { Musculoskeletal } \\
\text { pain (marked on } \\
\text { a drawing with } \\
\text { predefined body } \\
\text { regions) for more } \\
\text { than } 1 \text { week during } \\
\text { the last month }\end{array}$ & $\begin{array}{l}\text { Self } \\
\text { report }\end{array}$ & $\begin{array}{l}\text { One } \\
\text { month }\end{array}$ & $60-69$ & 8.8 & 15.7 & & $\mathrm{H}$ \\
\hline \multirow{3}{*}{$\begin{array}{l}\text { Thomas [87] } \\
2004 \text { UK }\end{array}$} & \multirow{3}{*}{$\begin{array}{l}\text { (50+ yo), all patients } \\
\text { from three GPs } \\
\text { from the North } \\
\text { Staffordshire } \\
\text { Primary Care } \\
\text { Research Consortium } \\
\text { (the NorStOP). } \\
\text { Postal } \\
\text { questionnaire }\end{array}$} & \multirow[t]{3}{*}{7878} & \multirow[t]{3}{*}{$?$} & \multirow[t]{3}{*}{$?$} & \multirow[t]{3}{*}{70} & \multirow{3}{*}{$\begin{array}{l}\text { "In the past } 4 \text { weeks } \\
\text { have you had pain that } \\
\text { has lasted for one day } \\
\text { or longer in any } \\
\text { part of your body?" } \\
\text { [supplemented by } \\
\text { a full body } \\
\text { manikin] }\end{array}$} & \multirow{3}{*}{$\begin{array}{l}\text { Self } \\
\text { report }\end{array}$} & \multirow{3}{*}{$\begin{array}{l}\text { One } \\
\text { month }\end{array}$} & $60-69$ & & & 37.7 & \multirow[t]{3}{*}{$u$} \\
\hline & & & & & & & & & $70-79$ & & & 35.4 & \\
\hline & & & & & & & & & \multicolumn{3}{|l|}{$80+$} & 37.6 & \\
\hline $\begin{array}{l}\text { Yaron [86] } \\
2011 \text { Israel }\end{array}$ & $\begin{array}{l}\text { 2002, 2006, 2008, } \\
\text { (20+ yo), stratified } \\
\text { sample drawn from } \\
\text { a telephone database } \\
\text { on different } \\
\text { population sectors. } \\
\text { Telephone } \\
\text { interview + } \\
\text { questionnaire }\end{array}$ & 2520 & $47.2 \%$ & $52.8 \%$ & $59-66$ & $\begin{array}{l}\text { The Community } \\
\text { Oriented Program } \\
\text { for the Control of } \\
\text { Rheumatic } \\
\text { Diseases } \\
\text { core } \\
\text { questionnaire } \\
\text { (CCQ): "In the } \\
\text { past } 7 \text { days have } \\
\text { you experienced pain } \\
\text { in any of the } \\
\text { following sites: } \\
\text { [knees]?" }\end{array}$ & $\begin{array}{l}\text { Self } \\
\text { report }\end{array}$ & $\begin{array}{l}\text { One } \\
\text { week }\end{array}$ & $61+$ & & & 63.9 & U \\
\hline \multirow{2}{*}{$\begin{array}{l}\text { Jordan [27] } \\
2007 \text { USA }\end{array}$} & \multirow[b]{2}{*}{$\begin{array}{l}\text { 1991-7, (45+ yo), } \\
\text { stratified simple } \\
\text { random sampling } \\
\text { of streets as primary } \\
\text { sampling units and } \\
\text { stratified subsampling } \\
\text { of Caucasian women } \\
\text { age } 65 \text { years or } \\
\text { older residents of } \\
\text { one of } 6 \text { townships } \\
\text { (the Johnston }\end{array}$} & \multirow[t]{2}{*}{3690} & \multirow[t]{2}{*}{$?$} & \multirow[t]{2}{*}{$?$} & \multirow[t]{2}{*}{72} & \multirow{2}{*}{$\begin{array}{l}\text { "On most days, } \\
\text { do you have pain, } \\
\text { aching, or stiffness } \\
\text { in your knee?" }\end{array}$} & \multirow{2}{*}{$\begin{array}{l}\text { Self } \\
\text { report }\end{array}$} & \multirow[t]{2}{*}{ Point } & \multirow{2}{*}{\multicolumn{3}{|c|}{$65-74$}} & $49(46.1-51.9)$ & U \\
\hline & & & & & & & & & & & & $56.6(52.7-60.4)$ & \\
\hline
\end{tabular}


Table 12 Description of studies on knee pain (Continued)

\begin{tabular}{|c|c|c|c|c|c|c|c|c|c|c|c|c|}
\hline & $\begin{array}{l}\text { County } \\
\text { Osteoarthritis } \\
\text { Project). Home } \\
\text { interview + clinical } \\
\text { examination } \\
\text { (local clinic) }\end{array}$ & & & & & & & & & & & \\
\hline $\begin{array}{l}\text { Picavet [78] } \\
2003 \text { The } \\
\text { Netherlands }\end{array}$ & $\begin{array}{l}\text { 1998, }(25+\text { yo), } \\
\text { stratified random } \\
\text { sample taken from } \\
\text { the population } \\
\text { register (the DMC3- } \\
\text { study). Postal } \\
\text { questionnaire }\end{array}$ & 3664 & $44.8 \%$ & $55.2 \%$ & 46 & $\begin{array}{l}\text { [Knee] pain } \\
\text { during the } \\
\text { survey }\end{array}$ & Self report & Point & $65+$ & $16.2(13.2-19.2)$ & $27.6(23.9-31.3)$ & L \\
\hline
\end{tabular}

Osteoarthritis

during the

qudy). Postal

Prevalence estimates without decimals are obtained from figures/graphs in the article and should be interpreted with caution.

I: Interview, Q: Questionnaire; E: Examination, R: Register. L: Low, U: Unclear, H: High. 
Table 13 Description of studies on ankle and foot pain

\begin{tabular}{|c|c|c|c|c|c|c|c|c|c|c|c|c|c|c|}
\hline & \multirow{2}{*}{$\begin{array}{l}\text { First author } \\
\text { Publ. year } \\
\text { Country }\end{array}$} & \multirow{2}{*}{$\begin{array}{l}\text { Study design / } \\
\text { Population / Method } \\
\text { of collection }\end{array}$} & \multicolumn{3}{|c|}{ Sample size } & \multirow{2}{*}{$\begin{array}{l}\text { Crude } \\
\text { response } \\
\text { rate }(\%)\end{array}$} & \multirow{2}{*}{$\begin{array}{l}\text { Outcome } \\
\text { definition }\end{array}$} & \multirow{2}{*}{$\begin{array}{l}\text { Outcome } \\
\text { assessment } \\
\text { method }\end{array}$} & \multirow{2}{*}{$\begin{array}{l}\text { Prevalence } \\
\text { period }\end{array}$} & \multirow{2}{*}{ Age } & \multicolumn{3}{|c|}{ Prevalence* $(95 \% \mathrm{Cl})$} & \multirow{2}{*}{$\begin{array}{l}\text { Risk } \\
\text { of } \\
\text { bias }\end{array}$} \\
\hline & & & Total & $M$ & $F$ & & & & & & $M$ & $F$ & Total & \\
\hline $\begin{array}{l}\text { Ankle } \\
\text { pain }\end{array}$ & $\begin{array}{l}\text { Dunn [99] } \\
2004 \text { UK }\end{array}$ & $\begin{array}{l}2001-2,(65+\text { yo), } \\
\text { individuals born on } \\
\text { or before } \\
\text { July } 31,1935 \text { and } \\
\text { residing in } \\
\text { Springfield, } \\
\text { identified by } \\
\text { Medicare } \\
\text { beneficiary files } \\
\text { and the } \\
\text { Springfield town } \\
\text { census. } \\
\text { Interview + } \\
\text { examination } \\
\text { (home) }\end{array}$ & 784 & 339 & 445 & 10 & $\begin{array}{l}\text { pain or discomfort in } \\
\text { any of their joints } \\
\text { on most days } \\
\text { during the } \\
\text { past } 4 \text { weeks }\end{array}$ & $\begin{array}{l}\text { Self } \\
\text { report }\end{array}$ & $\begin{array}{l}\text { One } \\
\text { month }\end{array}$ & $75+$ & 14.1 & 15.3 & 14.9 & $U$ \\
\hline $\begin{array}{l}\text { Ankle } \\
\text { pain }\end{array}$ & $\begin{array}{l}\text { Yaron [86] } \\
2011 \text { Israel }\end{array}$ & $\begin{array}{l}\text { 2002, 2006, 2008, } \\
\text { (20+ yo), stratified } \\
\text { sample drawn from } \\
\text { a telephone } \\
\text { database on } \\
\text { different population } \\
\text { sectors. Telephone } \\
\text { interview + } \\
\text { questionnaire }\end{array}$ & 2520 & $47.2 \%$ & $52.8 \%$ & $59-66$ & $\begin{array}{l}\text { The Community } \\
\text { Oriented Program } \\
\text { for the Control of } \\
\text { Rheumatic Diseases } \\
\text { core questionnaire } \\
\text { (CCQ): "In the past } \\
7 \text { days have you } \\
\text { experienced pain } \\
\text { in any of the } \\
\text { following sites: } \\
\text { [ankles]?" }\end{array}$ & $\begin{array}{l}\text { Self } \\
\text { report }\end{array}$ & $\begin{array}{l}\text { One } \\
\text { week }\end{array}$ & $61+$ & & & 35.9 & U \\
\hline $\begin{array}{l}\text { Ankle } \\
\text { pain }\end{array}$ & $\begin{array}{l}\text { Picavet [78] } \\
2003 \text { The } \\
\text { Netherlands }\end{array}$ & $\begin{array}{l}\text { 1998, }(25+\text { yo), } \\
\text { stratified random } \\
\text { sample taken from } \\
\text { the population } \\
\text { register (the DMC3- } \\
\text { study). Postal } \\
\text { questionnaire }\end{array}$ & 3664 & $44.8 \%$ & $55.2 \%$ & 46 & $\begin{array}{l}\text { [Ankle] pain } \\
\text { during the } \\
\text { survey }\end{array}$ & $\begin{array}{l}\text { Self } \\
\text { report }\end{array}$ & Point & $65+$ & $4.6(2.9-6.3)$ & $9.8(7.4-12.2)$ & & L \\
\hline \multirow{2}{*}{$\begin{array}{l}\text { Ankle/ } \\
\text { foot } \\
\text { pain }\end{array}$} & \multirow{2}{*}{$\begin{array}{l}\text { Parsons [77] } \\
2007 \text { UK }\end{array}$} & \multirow[b]{2}{*}{$\begin{array}{l}\text { 2001-3, (18+ yo), } \\
\text { random samples } \\
\text { from } 16 \text { Medical } \\
\text { Research Council } \\
\text { General Practice } \\
\text { Research Framework } \\
\text { practices, South } \\
\text { East quadrant of } \\
\text { the UK. Postal } \\
\text { questionnaire }\end{array}$} & \multirow[t]{2}{*}{2501} & \multirow[t]{2}{*}{1347} & \multirow[t]{2}{*}{1154} & \multirow[t]{2}{*}{47} & \multirow[b]{2}{*}{$\begin{array}{l}\text { The Chronic Pain } \\
\text { Grade: Any 'pain } \\
\text { which has lasted } \\
\text { for } 3 \text { months or } \\
\text { longer and } \\
\text { currently } \\
\text { troubles } \\
\text { respondents either } \\
\text { all of the time } \\
\text { or on and off' }\end{array}$} & \multirow{2}{*}{$\begin{array}{l}\text { Self } \\
\text { report }\end{array}$} & \multirow{2}{*}{$\begin{array}{l}\text { Three } \\
\text { months }\end{array}$} & $65-74$ & & & 4 & \multirow[t]{2}{*}{ U } \\
\hline & & & & & & & & & & $75-101$ & & & 5 & \\
\hline
\end{tabular}


Table 13 Description of studies on ankle and foot pain (Continued)

\begin{tabular}{|c|c|c|c|c|c|c|c|c|c|c|c|c|c|c|}
\hline \multirow{2}{*}{$\begin{array}{l}\text { Foot } \\
\text { pain }\end{array}$} & \multirow{2}{*}{$\begin{array}{l}\text { Peat [92] } \\
2006 \text { UK }\end{array}$} & \multirow[b]{2}{*}{$\begin{array}{l}2002,(50+\text { yo), all } \\
\text { community-dwelling } \\
\text { adults registered } \\
\text { with } 3 \text { general } \\
\text { practices in North } \\
\text { Staffordshire (The } \\
\text { NorStOP). Postal } \\
\text { questionnaire }\end{array}$} & \multirow[t]{2}{*}{2429} & \multirow[t]{2}{*}{1005} & \multirow[t]{2}{*}{1424} & \multirow[t]{2}{*}{22} & \multirow{2}{*}{$\begin{array}{l}\text { The Regional } \\
\text { Pains Survey, } \\
\text { containing the Foot } \\
\text { Disability Index } \\
\text { (the FDI- } \\
\text { FOOT) }\end{array}$} & \multirow{2}{*}{$\begin{array}{l}\text { Self } \\
\text { report }\end{array}$} & \multirow{2}{*}{$\begin{array}{l}\text { One } \\
\text { year }\end{array}$} & $65-74$ & 45 & \multicolumn{2}{|l|}{58} & \multirow[t]{2}{*}{ U } \\
\hline & & & & & & & & & & $75+$ & 51 & 55 & & \\
\hline \multirow{2}{*}{$\begin{array}{l}\text { Foot } \\
\text { pain }\end{array}$} & \multirow{2}{*}{$\begin{array}{l}\text { Keenan [73] } \\
2006 \text { UK }\end{array}$} & \multirow[b]{2}{*}{$\begin{array}{l}\text { 1993, (55+ yo), a } \\
\text { two-stage random } \\
\text { sample from the } \\
\text { North Yorkshire } \\
\text { Family Health } \\
\text { Services Authority. } \\
\text { Postal } \\
\text { questionnaire }\end{array}$} & \multirow[t]{2}{*}{16222} & \multirow[t]{2}{*}{$?$} & \multirow[t]{2}{*}{$?$} & \multirow[t]{2}{*}{86} & \multirow[b]{2}{*}{$\begin{array}{l}\text { Any swelling, pain, } \\
\text { or stiffness in any } \\
\text { of their joints that } \\
\text { lasted }>6 \text { weeks in } \\
\text { the previous } 3 \\
\text { months (identified } \\
\text { on a manikin) }\end{array}$} & \multirow{2}{*}{$\begin{array}{l}\text { Self } \\
\text { report }\end{array}$} & \multirow{2}{*}{$\begin{array}{l}\text { Three } \\
\text { months }\end{array}$} & $65-74$ & $14.1(12.8-15.4)$ & $20.7(19.2-22.2)$ & $17.7(16.3-19.1)$ & 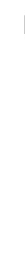 \\
\hline & & & & & & & & & & $75+$ & $14.0(12.7-15.3)$ & $26.9(25.3-28.3)$ & $22.5(21.0-24.0)$ & \\
\hline \multirow{3}{*}{$\begin{array}{l}\text { Foot } \\
\text { pain }\end{array}$} & \multirow{3}{*}{$\begin{array}{l}\text { Miro [75] } \\
2007 \text { Spain }\end{array}$} & \multirow{3}{*}{$\begin{array}{l}\text { (65+ yo), stratified } \\
\text { random sample } \\
\text { taken from the } \\
\text { population census } \\
\text { obtained from the } \\
\text { Catalan Statistics } \\
\text { Institute, Catalonia. } \\
\text { Interview + } \\
\text { questionnaire } \\
\text { (local primary } \\
\text { care centre) }\end{array}$} & \multirow[t]{3}{*}{592} & \multirow[t]{3}{*}{274} & \multirow[t]{3}{*}{318} & \multirow[t]{3}{*}{99} & \multirow{3}{*}{$\begin{array}{l}\text { The Chronic Pain } \\
\text { Grade: "In the past } \\
3 \text { months have you } \\
\text { had pain that has } \\
\text { lasted for one day } \\
\text { or longer in any part } \\
\text { of your body?" }\end{array}$} & \multirow{3}{*}{$\begin{array}{l}\text { Self } \\
\text { report }\end{array}$} & \multirow{3}{*}{$\begin{array}{l}\text { Three } \\
\text { months }\end{array}$} & $65-74$ & & & 37.4 & \multirow[t]{2}{*}{ 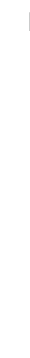 } \\
\hline & & & & & & & & & & $75-84$ & & & 44.1 & \\
\hline & & & & & & & & & & $85+$ & & & 55.8 & \\
\hline $\begin{array}{l}\text { Foot } \\
\text { pain }\end{array}$ & $\begin{array}{l}\text { Mickle [97] } \\
2010 \text { Australia }\end{array}$ & $\begin{array}{l}(60+\text { yo), from } 16 \\
\text { randomly selected } \\
\text { federal electorates } \\
\text { in Sydney and } \\
\text { Illawarra statistical } \\
\text { regions, New } \\
\text { South Wales. } \\
\text { Questionnaire }\end{array}$ & 312 & 158 & 154 & 16 & $\begin{array}{l}\text { The Manchester } \\
\text { Foot Pain and } \\
\text { Disability Index } \\
\text { (MFPDI) } \geq 1\end{array}$ & $\begin{array}{l}\text { Self } \\
\text { report }\end{array}$ & $\begin{array}{l}\text { One } \\
\text { month }\end{array}$ & $60+$ & & & 50 & $\mathrm{H}$ \\
\hline $\begin{array}{l}\text { Foot } \\
\text { pain }\end{array}$ & $\begin{array}{l}\text { Mølgaard [98] } \\
2010 \text { Denmark }\end{array}$ & $\begin{array}{l}\text { 2005, (18-80 yo), } \\
\text { random sample from } \\
\text { the Danish Civil } \\
\text { Registration System } \\
\text { of the Aalborg } \\
\text { municipality. } \\
\text { Postal } \\
\text { questionnaire }\end{array}$ & 1671 & 807 & 864 & 80 & $\begin{array}{l}\text { "Have you within the } \\
\text { last month had pain } \\
\text { in your feet which } \\
\text { lasted more than } \\
\text { one day?" }\end{array}$ & $\begin{array}{l}\text { Self } \\
\text { report }\end{array}$ & $\begin{array}{l}\text { One } \\
\text { month }\end{array}$ & $60-80$ & & & 28.6 & L \\
\hline \multirow{3}{*}{$\begin{array}{l}\text { Foot } \\
\text { pain }\end{array}$} & \multirow{3}{*}{$\begin{array}{l}\text { Thomas [87] } \\
2004 \text { UK }\end{array}$} & (50+ yo), all & 7878 & $?$ & $?$ & 70 & "In the past 4 weeks & Self & One & $60-69$ & & & 23.5 & $U$ \\
\hline & & $\begin{array}{l}\text { patients from } \\
\text { three GPs from }\end{array}$ & & & & & $\begin{array}{l}\text { have you had pain } \\
\text { that has lasted for }\end{array}$ & report & month & 70-79 & & & 22.5 & \\
\hline & & $\begin{array}{l}\text { the North } \\
\text { Staffordshire } \\
\text { Primary Care }\end{array}$ & & & & & $\begin{array}{l}\text { one day or longer } \\
\text { in any part of your } \\
\text { body?" [supplemented }\end{array}$ & & & $80+$ & & & 19.5 & \\
\hline
\end{tabular}

body?" [supplemented 
Table 13 Description of studies on ankle and foot pain (Continued)

\begin{tabular}{|c|c|c|c|c|c|c|c|c|c|c|c|c|c|c|}
\hline & & $\begin{array}{l}\text { Research } \\
\text { Consortium (the } \\
\text { NorStOP). Postal } \\
\text { questionnaire }\end{array}$ & & & & & $\begin{array}{l}\text { by a full body } \\
\text { manikin] }\end{array}$ & & & & & & & \\
\hline $\begin{array}{l}\text { Foot } \\
\text { pain }\end{array}$ & $\begin{array}{l}\text { Badlissi [95] } \\
2005 \text { USA }\end{array}$ & $\begin{array}{l}2001-2,(65+\text { yo), } \\
\text { individuals born } \\
\text { on or before } \\
\text { July } 31,1935 \text { and } \\
\text { residing in } \\
\text { Springfield, } \\
\text { identified by } \\
\text { Medicare } \\
\text { beneficiary files } \\
\text { and the Springfield } \\
\text { town census. } \\
\text { Interview + } \\
\text { questionnaire } \\
\text { (telephone) + } \\
\text { examination } \\
\text { (home visit) }\end{array}$ & 784 & 339 & 445 & 10 & $\begin{array}{l}\text { Aches or pains in your } \\
\text { feet past week or any } \\
\text { foot pain or } \\
\text { discomfort on } \\
\text { most days during } \\
\text { the past four } \\
\text { weeks }\end{array}$ & $\begin{array}{l}\text { Self } \\
\text { report }\end{array}$ & $\begin{array}{l}\text { One } \\
\text { month }\end{array}$ & $65+$ & & & 41.6 & $\mathrm{H}$ \\
\hline $\begin{array}{l}\text { Foot } \\
\text { pain }\end{array}$ & $\begin{array}{l}\text { Menz [96] } \\
2005 \text { Australia }\end{array}$ & $\begin{array}{l}\text { (62-92 yo), combined } \\
\text { independent units } \\
\text { and serviced } \\
\text { apartments in } \\
\text { retirement village. } \\
\text { Questionnaire + } \\
\text { examination } \\
\text { (home) }\end{array}$ & 176 & 56 & 120 & $?$ & $\begin{array}{l}\text { Subjects were } \\
\text { asked whether they } \\
\text { suffered from } \\
\text { painful feet }\end{array}$ & $\begin{array}{l}\text { Self } \\
\text { report }\end{array}$ & Point & $62-92$ & 14 & 28 & 24 & L \\
\hline $\begin{array}{l}\text { Foot } \\
\text { pain }\end{array}$ & $\begin{array}{l}\text { Picavet [78] } \\
2003 \text { The } \\
\text { Netherlands }\end{array}$ & $\begin{array}{l}\text { 1998, }(25+\text { yo), } \\
\text { stratified random } \\
\text { sample taken from } \\
\text { the population } \\
\text { register (the DMC3- } \\
\text { study). Postal } \\
\text { questionnaire }\end{array}$ & 3664 & $44.8 \%$ & $55.2 \%$ & 46 & $\begin{array}{l}\text { [Foot] pain during } \\
\text { the survey }\end{array}$ & $\begin{array}{l}\text { Self } \\
\text { report }\end{array}$ & Point & $65+$ & $8.9(6.3-11.2)$ & $11.8(9.2-14.4)$ & & L \\
\hline
\end{tabular}

*Prevalence estimates without decimals are obtained from figures/graphs in the article and should be interpreted with caution.

I: Interview, Q: Questionnaire; E: Examination, R: Register. L: Low, U: Unclear, H: High. 
Two studies with similar designs and definitions reported that $23 \%-29 \%$ of $60-80$ year olds had pain in their feet during the past month $[87,98]$. In contrast, two other similar studies on point prevalence showed greater variations (65+ men: 9\%-14\%; women: $12 \%-28 \%)$ [78,96]. Otherwise, great variations in prevalence were found, for the same reasons as described under the wrist/hand pain section. In all the studies reporting gender prevalences, women suffered more from ankle and/or foot pain than men $[73,78,92,96,99]$. In two studies, foot pain increased with age $[73,75]$, but dropped in another study [87].

\section{Musculoskeletal co-morbidity}

Information on multiple/widespread MSK conditions in the elderly population was extracted from 15 studies [30,59,68,72,75,78,82,84,86,87,89,91-93,100].

In a Danish elderly population (70-120 year olds), concurrent neck and BP was found in 13\% of women and $8 \%$ of men [68]. The same findings were reported in the USA, where $9 \%$ of $65+$ year olds had both NP and LBP [82]. Jacobs et al. reported an almost twofold increase in concurrent joint pain among older people (70 and 77 year olds) with chronic BP (59\% and $74 \%$ respectively) compared to those without chronic BP [72].

Widespread pain was reported in the study by Natvig et al., where $14-15 \%$ of Norwegian people aged 6486 years had additional MSK pain (from either shoulders, elbows, hands/wrists, upper back, lower back, hips, knees, or ankles/feet) [100]. In Sweden, between $4 \%$ and $6 \%$ of men aged $65-74$ with upper extremity pain also reported either NP, LBP, or lower extremity pain, whereas in women the reported prevalence was about three times higher (15\%-17\%) [89]. According to Vogt et al., $14 \%$ of 70 to 79 year old Americans reported concurrent MSK pain in at least four sites [84]. In the UK, three studies on multiple pain sites showed varying results among 65+ year olds, which may be due to different definitions [91-93]. According to Dawson et al., 11\% of the older adults had both hip and knee pain [91]. Croft et al. reported slightly higher estimates (26\%-33\%) but included the whole body [93]. In the study by Peat et al., 40\% had more than one painful joint in the lower extremity [92]. More widespread pain (up to 44 pain sites) was reported by $12 \%-16 \%$ of women and by $7 \%$ $13 \%$ of men aged 60 and over [87]. In Italy, "polyarticular peripheral joint pain" was reported in $28 \%$ in the same age group (65+) [30]. In a Dutch study, multiple MSK pain sites were present in roughly $28 \%$ of men and in $46 \%$ of women aged 65 and over [78].

Other studies report several MSK pain sites in more than half of the elderly people, which indicates overlapping MSK symptoms $[59,75,86]$. In a South Korean elderly population $(65+)$, more than half reported both upper extremity pain as well as LBP and/or lower extremity pain [59]. Similarly, in an Israeli population of elderly people aged 61 and over, more than half reported LBP, NP, knee and shoulder pain [86]. Furthermore, at least a third of these people also reported other peripheral joint pain sites. Finally, in a Spanish study, people aged 65 and over had on average four MSK pain sites [75]. Unfortunately, it is not possible to determine how many of these suffered from multiple pain sites. Thus, based on these three studies, a high degree of overlapping/concurrent MSK pain sites must be present $[59,75,86]$.

\section{In summary}

- The prevalence of MSK conditions remains high even in old age regardless of the type of complaint.

- Women typically report problems more often than men, regardless of the MSK condition.

- The prevalence of MSK complaints typically drops slightly in the oldest age group (i.e. 80+ year olds), except for OP where all studies report an age related increase.

- Widespread/concurrent MSK pain is very common among elderly people, affecting every second or third elderly person.

\section{Discussion}

\section{Summary of evidence}

In this review a great variation in prevalence of MSK disorders in older people were found. The most likely reasons for these differences are: 1) different pain definitions, 2) different prevalence periods, 3) different age intervals, and 4) the prevalence estimates were either divided by gender or only reported as a total prevalence estimate. Thus, it is impossible to determine any overall estimates on the prevalence of MSK problems in the elderly population.

Nevertheless, some general observations can be drawn from this review that needs to be discussed. Musculoskeletal disorders remain prevalent in the elderly population. Especially, OA is very common among elderly people, followed by knee pain, BP, and for women also OP. Pain mechanisms in the older population are poorly understood, but it is generally believed that pain at younger ages continues in the older ages [101]. Thus, pain in the elderly should be regarded as a continuum of pain from earlier years [101].

Women tend to report MSK pain significantly more often than men in almost all studies. This gender difference in pain reporting is well known, but the reason for this is probably multifactorial with both biological and psychosocial underlying mechanisms. These different pain mechanisms are beyond the scope of this paper to 
discuss in detail, but are presented in a review by Fillinghim et al. [102].

There is a general trend that prevalence estimates either remain fairly constant with increasing age or that they drop slightly in the oldest people, typically from 80 years of age and onwards. An exception from this is OP, where a steady increase is reported with increasing age.

There are several potential explanations for this decline in pain reporting with age. It may simply be a general birth cohort effect which may reflect both cultural and public health related differences between for example 40 year olds and 80 year olds [103]. This potential cohort effect may be more pronounced in crosssectional studies, which were the only included studies in this review. A parallel to this may be that pain is accepted by the elderly as part of becoming old [104]. In other words, pain becomes a natural part of their life and therefore become less disturbing or simply ignored. It is also known that pressure pain decreases with age [105]. Finally, a decline in pain prevalences in the oldest old could be explained by a "survival of the fittest" phenomenon [103]. However, MSK pain itself does not lead to premature mortality per se [106-108]. Furthermore, this "biological elite" phenomenon is probably slowly diminishing as health and living standards in the World is generally improving and thus, more people are living longer and generally at better health

Finally, there is a considerable degree of overlapping MSK symptoms as approximately every second or third elderly have widespread MSK pain. This trend is most likely part of a continuum from widespread pain at younger ages as previously mentioned [101].

\section{Comparisons with other reviews}

To our knowledge, no previous systematic literature reviews on a broader range of MSK conditions in elderly populations exist. However, a few reviews on some of our MSK conditions in the elderly populations were identified. Woolf and Pfleger reported high prevalence estimates in the elderly people for OA, RA, OP, and LBP in the developed countries [4]. In all four MSK diseases, the same age related increase in prevalence was found in their review, except for LBP where it remained fairly constant.

A literature review on LBP before 2000 found only 12 prevalence estimates specifically on elderly populations, but the authors were unable to make any general estimates mainly because of the different (or lack of) LBP definitions as well as the varying age intervals [109]. In a more recent LBP review published in 2006 on age related changes, concluded that "benign" LBP decreased with age, but that more severe LBP increased with age [110]. Due to the heterogeneity of these studies and the aim of their review, no attempt was made to provide any general LBP prevalence estimates.

Luime et al. published a review in 2004 on shoulder pain [111]. The point prevalence on subjects $<70$ ranging 7\%-27\% was very similar for subjects older than 70 (12-26\%), but this may be due to the varying pain definitions.

Dagenais et al. found a steady increase in hip OA with increasing age, ranging from 5\% (60-64 year olds) to $14 \%$ (85+ year olds), and being more prevalent in women [112].

It is impossible to compare our results with the abovementioned reviews, as they too fail to provide pooled estimates due to the high degree of heterogeneity across the included studies. Nevertheless, a general increase in prevalence with age and a gender difference were reported in all reviews, which is in accordance with our own findings.

\section{Methodological issues}

The heterogeneity of pain definitions is already a well known problem, but undoubtedly, researchers have many good reasons for why they use a specific and perhaps unique pain definition. Unfortunately, this makes it impossible to draw any general conclusions based on the currently available literature. However, it would be recommendable if authors would at least report one or two additional standardised measures, such as the questions from the standardised Nordic questionnaire on musculoskeletal pain [113]. Although, journals restrict the sizes of their papers by limiting the number of words or tables and hence, decreasing the amount of information available from the studies, it is becoming more and more common to have supplementary tables published via the publishing journal's website. Such tables could include valuable information on gender specific and total prevalence estimates for future reviews to calculate pooled prevalence estimates.

It also needs mentioning that nearly twice as many prevalence estimates could have been obtained from 82 additional studies, if only authors had reported age specific estimates. So, just like the standardisation of pain definitions is warranted, standardisation of age interval reporting would also be preferable. This way, more information on age related changes from the current literature could easily have been obtained.

In this review, we found that many authors state that their results are representative of the general population. However, only few actually document this. While many do their best at obtaining a random and representative target sample from the background population, an actual non-response analysis is rarely performed. For this reason, the risk of bias of the majority of the studies (65\%) was deemed unclear. Studies were generally judged as 
having an "unclear" risk of bias because information was missing in the study description. In other words, the external validity of these studies is questionable, which is essential in epidemiological studies. It is therefore important to either report and/or adjust for non-response bias in future studies.

\section{Strengths and limitations of this review}

Just like our included studies, our review has also some limitations that need to be addressed. We only included one electronic database (Pubmed) and thus, may have missed some relevant articles. Based on other reviews on similar MSK conditions, who have included other electronic databases (i.e. EMBASE, CINAHL, etc.), we may have missed between zero and $12 \%$ potentially relevant articles [109-112]. However, given the large heterogeneity and therefore lack of proper summary prevalence estimates, we doubt any missed articles would have had any major impact on our results. Our search strategy was also limited to the elderly population through $\mathrm{MeSH}$ terms. This may have lead to exclusion of some studies if for some reason they were not properly indexed in Pubmed. As only English language articles were included, any articles published in national non-English medical journals are missing in our literature review. Finally, the selection of articles was only conducted by one author, thus, there is a risk of missing potentially relevant articles. According to Edwards et al., an average of 9\% of relevant articles may be missed (ranging between 0 and 32\%) [114]. Thus, on average we may have missed approximately 8 articles.

The results from the included epidemiological studies must be viewed in light of the quality of these studies which depends on both the internal validity and if the results can be extrapolated to the background population (i.e. the external validity). In this review, the risk of bias rather than the quality of the studies were used as we wished to determine if the results were "believable" and not just if the "reporting" was satisfactory. The risk of bias assessment on randomised clinical trials is also recommended by the Cochrane Collaboration [8] and recently a set of risk of bias items were developed by Viswanathan et al. [9] which allowed us to design an assessment sheet well suited for our needs. However, assessing the risk of bias demands a high degree of judgement, is more time consuming, and may result in greater variability of interpretations of the studies $[9,115]$. Therefore, no attempt at adjusting the prevalence estimates based on the risk of bias judgment was made. Instead, we leave it up to the readers to decide on how to utilise our risk of bias judgments.

Because MSK pain may be reported as part of a larger health related publication and because a wide set of MSK conditions were included in our review, it was necessary to have rather broad search strategy. This in turn, resulted in a very large number of hits that had to be perused to seek for any potentially relevant articles. While the search may have been fairly sensitive in catching relevant articles it cannot be considered to be very specific. This becomes clear as less than $4 \%$ of the initial search results were retrieved and only $46 \%$ of those included. We did not attempt to specify the literature search any further as some of the included articles would have been missed, especially those articles where the reporting of MSK conditions are "secondary" findings.

Another limitation is the choice of only investigating the prevalence of MSK disorders among elderly people and, hence, excluding information on burden and costof-illness of these MSK conditions. Clearly, the presence of pain does not reflect how MSK problems affect older people on a daily basis. However, in the 2003 WHO report, Woolf and Pfleger reported that MSK conditions have a major societal impact in terms of reduced work disability, which would affect the "younger" elderly people aged 60-65, and result in an increased use of health care services [4]. Finally, with increasing OP, there is a high risk of fracture incidences. As most MSK conditions remain fairly common in the elderly populations and as the number of elderly people increases in the future, the socioeconomic burden of MSK in the elderly population will also increase. Thus, there will be a further need for health care professionals to deal with chronic MSK conditions among the elderly people.

\section{Future perspectives}

This review has looked at the prevalence of a series of musculoskeletal conditions in the elderly population and will serve not only as a reference for future studies, but also as a guide for clinicians in general. Firstly, a larger population of geriatric patients must be expected in the future and thus calls for more attention on developing optimal geriatric patient management protocols. Secondly, it is important for a person to maintain a sufficient functional capacity in order to maintain an active life at older age [3]. In other words, political programmes as well as primary and secondary health care programmes accommodated to the future needs are necessary in order to maintain (or ideally improve) the quality of life in the elderly population.

\section{Conclusions}

No overall estimate on the prevalence of MSK problems in the elderly population can be determined due to the heterogeneity of the studies. However, MSK disorders are common in the elderly population and women have more often MSK problems than men. There is a general trend that prevalence estimates either remain fairly 
constant or increase slightly with increasing age. However, for many MSK conditions, there is a slight decrease among the oldest $(80+)$ people. Finally, many elderly people report multiple MSK pain sites.

\section{Additional files}

\section{Additional file 1: List of developed countries included in this} literature review. Included countries in this review based on advanced economies according to the International Monetary Foundation.

\section{Additional file 2: Search strategy - Pubmed.org.}

Additional file 3: Overview of excluded articles. All retrieved articles that were initially considered of relevance, but subsequently excluded because inclusion/exclusion criteria were not fulfilled.

Additional file 4: Risk of bias for all included studies. All included studies were assessed for potential risk of bias.

\section{Competing interests}

The authors declare that they have no competing interests.

\section{Authors' contributions}

RF planned the design of the study, conducted the literature search and wrote the initial draft of the manuscript. AR cross checked the extracted data including the risk of bias assessments. Both authors participated in writing the final manuscript.

\section{Acknowledgements}

RF was partially supported by the Danish Foundation for Post-graduate Chiropractic Education and Research.

\section{Author details}

'The Research Department, the Spine Centre of Southern Denmark, Hospital Lillebaelt, Middelfart, Denmark. ${ }^{2}$ Private practice, Praxis fuer Chiropraktik Wolfsburg, Wolfsburg, Germany.

Received: 1 July 2012 Accepted: 18 September 2012 Published: 24 September 2012

\section{References}

1. United Nations DoEaSA: World Population Ageing 2009. New York: 2010.

2. The Disease Control Priorities Project: Global Burden of Disease and Risk Factors. Washington: 2006.

3. World Health Organization: Active Ageing - A Policy Framework. Geneva: 2002.

4. Woolf AD, Pfleger B: Burden of major musculoskeletal conditions. Bulletin of the World Health Organization 2003, 81:646-656.

5. Woolf AD: The bone and joint decade 2000-2010. Ann Rheum Dis 2000, 59:81-82.

6. The Bone and Joint Decade's Musculoskeletal Portal. http://www.boneandjointdecade.org/.

7. The International Monetary Fund - Database - WEO Groups and Aggregate Information. www.imf.org/external/pubs/ft/weo/2011/02/weodata/groups. $\mathrm{htm}$.

8. Higgins JPT, Green S (Eds): Cochrane Handbook for Systematic Reviews of Interventions Version 5.1.0 [updated March 2011]. The Cochrane Collaboration; 2011.

9. Viswanathan M, Berkman ND: Development of the RTI item bank on risk of bias and precision of observational studies. J Clin Epidemiol 2011, 65:163-178.

10. Andrianakos A, Trontzas P, Christoyannis F, Kaskani E, Nikolia Z, Tavaniotou E, Georgountzos A, Krachtis P: Prevalence and management of rheumatoid arthritis in the general population of Greece-the ESORDIG study. Rheumatology (Oxford) 2006, 45:1549-1554.

11. Carmona L, Ballina J, Gabriel R, Laffon A: The burden of musculoskeletal diseases in the general population of Spain: results from a national survey. Ann Rheum Dis 2001, 60:1040-1045.
12. Collerton J, Davies $K$, Jagger C, Kingston A, Bond J, Eccles MP, Robinson LA, Martin-Ruiz C, von ZT, James OF: Health and disease in 85 year olds: baseline findings from the Newcastle 85+ cohort study. BMJ 2009, 339:b4904.

13. Englund M, Joud A, Geborek P, Felson DT, Jacobsson LT, Petersson IF: Prevalence and incidence of rheumatoid arthritis in southern Sweden 2008 and their relation to prescribed biologics. Rheumatology (Oxford) 2010, 49:1563-1569.

14. Hanova P, Pavelka K, Dostal C, Holcatova I, Pikhart H: Epidemiology of rheumatoid arthritis, juvenile idiopathic arthritis and gout in two regions of the Czech Republic in a descriptive population-based survey in 2002-2003. Clin Exp Rheumatol 2006, 24:499-507.

15. Laiho K, Tuomilehto J, Tilvis R: Prevalence of rheumatoid arthritis and musculoskeletal diseases in the elderly population. Rheumatol Int 2001, 20:85-87.

16. Neovius M, Simard JF, Askling J: Nationwide prevalence of rheumatoid arthritis and penetration of disease-modifying drugs in Sweden. Ann Rheum Dis 2011, 70:624-629.

17. Ollivier Y, Saraux A, Le GP: Prevalences of rheumatoid arthritis in Roman Catholic nuns and the general female population in Brittany, France: a pilot study. Clin Exp Rheumatol 2004, 22:759-762.

18. Picavet HS, Schouten JS: Musculoskeletal pain in the Netherlands: prevalences, consequences and risk groups, the DMC(3)-study. Pain 2003, 102:167-178.

19. Rasch EK, Hirsch R, Paulose-Ram R, Hochberg MC: Prevalence of rheumatoid arthritis in persons 60 years of age and older in the United States: effect of different methods of case classification. Arthritis Rheum 2003, 48:917-926.

20. Riise T, Jacobsen BK, Gran JT: Incidence and prevalence of rheumatoid arthritis in the county of Troms, northern Norway. J Rheumatol 2000, 27:1386-1389

21. Symmons $D$, Turner $G$, Webb R, Asten P, Barrett E, Lunt M, Scott D, Silman $A$ : The prevalence of rheumatoid arthritis in the United Kingdom: new estimates for a new century. Rheumatology (Oxford) 2002, 41:793-800.

22. Arnett FC, Edworthy SM, Bloch DA, McShane DJ, Fries JF, Cooper NS, Healey LA, Kaplan SR, Liang MH, Luthra HS, et al: The American Rheumatism Association 1987 revised criteria for the classification of rheumatoid arthritis. Arthritis Rheum 1988, 31:315-324.

23. Andrianakos AA, Kontelis LK, Karamitsos DG, Aslanidis SI, Georgountzos Al, Kaziolas GO, Pantelidou KV, Vafiadou EV, Dantis PC: Prevalence of symptomatic knee, hand, and hip osteoarthritis in Greece. The ESORDIG study. J Rheumatol 2006, 33:2507-2513.

24. Dillon CF, Hirsch R, Rasch EK, Gu Q: Symptomatic hand osteoarthritis in the United States: prevalence and functional impairment estimates from the third U.S. National Health and Nutrition Examination Survey, 1991-1994. Am J Phys Med Rehabil 2007, 86:12-21.

25. Fernandez-Lopez JC, Laffon A, Blanco FJ, Carmona L: Prevalence, risk factors, and impact of knee pain suggesting osteoarthritis in Spain. Clin Exp Rheumatol 2008, 26:324-332.

26. Haugen IK, Englund M, Aliabadi P, Niu J, Clancy M, Kvien TK, Felson DT: Prevalence, incidence and progression of hand osteoarthritis in the general population: the Framingham Osteoarthritis Study. Ann Rheum Dis 2011, 70:1581-1586

27. Jordan KP, Kadam UT, Hayward R, Porcheret M, Young C, Croft P: Annual consultation prevalence of regional musculoskeletal problems in primary care: an observational study. BMC Musculoskelet Disord 2010, 11:144.

28. Kim I, Kim HA, Seo YI, Song YW, Jeong JY, Kim DH: The prevalence of knee osteoarthritis in elderly community residents in Korea. J Korean Med Sci 2010, 25:293-298.

29. Kwok WY, Kloppenburg M, Rosendaal FR, van Meurs JB, Hofman A, Bierma-Zeinstra SM: Erosive hand osteoarthritis: its prevalence and clinical impact in the general population and symptomatic hand osteoarthritis. Ann Rheum Dis 2011, 70:1238-1242.

30. Mannoni A, Briganti MP, Di BM, Ferrucci L, Costanzo S, Serni U, Masotti G, Marchionni N: Epidemiological profile of symptomatic osteoarthritis in older adults: a population based study in Dicomano, Italy. Ann Rheum Dis 2003, 62:576-578.

31. Muraki S, Oka H, Akune T, Mabuchi A, En-Yo Y, Yoshida M, Saika A, Suzuki T, Yoshida $\mathrm{H}$, Ishibashi $\mathrm{H}$, et al: Prevalence of radiographic knee osteoarthritis and its association with knee pain in the elderly of 
Japanese population-based cohorts: the ROAD study. Osteoarthr Cartil 2009, 17:1137-1143.

32. Sudo A, Miyamoto N, Horikawa K, Urawa M, Yamakawa T, Yamada T, Uchida A: Prevalence and risk factors for knee osteoarthritis in elderly Japanese men and women. J Orthop Sci 2008, 13:413-418.

33. Yoshimura N, Muraki S, Oka H, Mabuchi A, En-Yo Y, Yoshida M, Saika A, Yoshida H, Suzuki T, Yamamoto S, et al: Prevalence of knee osteoarthritis, lumbar spondylosis, and osteoporosis in Japanese men and women: the research on osteoarthritis/osteoporosis against disability study. J Bone Miner Metab 2009, 27:620-628.

34. Yoshida S, Aoyagi K, Felson DT, Aliabadi P, Shindo H, Takemoto T: Comparison of the prevalence of radiographic osteoarthritis of the knee and hand between Japan and the United States. J Rheumatol 2002, 29:1454-1458

35. Yoshimura N, Muraki S, Oka H, Mabuchi A, Kinoshita H, Yosihda M, Kawaguchi H, Nakamura K, Akune T: Epidemiology of lumbar osteoporosis and osteoarthritis and their causal relationship-is osteoarthritis a predictor for osteoporosis or vice versa?: the Miyama study. Osteoporos Int 2009, 20:999-1008.

36. Zhang Y, Niu J, Kelly-Hayes M, Chaisson CE, Aliabadi P, Felson DT: Prevalence of symptomatic hand osteoarthritis and its impact on functional status among the elderly: The Framingham Study. Am J Epidemiol 2002, 156:1021-1027.

37. Mannoni A, Briganti MP, Di BM, Ferrucci L, Serni U, Masotti G, Marchionni N: Prevalence of symptomatic hand osteoarthritis in community-dwelling older persons: the ICARe Dicomano study. Insufficienza Cardiaca negli Anzizni Residenti a Dicomano. Osteoarthr Cartil 2000, 8(Suppl A):S11-S13.

38. Altman RD, Gold GE: Atlas of individual radiographic features in osteoarthritis, revised. Osteoarthr Cartil 2007, 15(Suppl A):A1-A56.

39. Kellgrenn JH, LAWRENCE JS: Radiological assessment of osteo-arthrosis. Ann Rheum Dis 1957, 16:494-502.

40. [No authors listed]: The Atlas of Standard Radiographs of Arthritis. Rheumatology (Oxford) 2005, 44(Suppl 4):iv46-iv72.

41. Andrianakos A, Trontzas P, Christoyannis F, Dantis P, Voudouris C, Georgountzos A, Kaziolas G, Vafiadou E, Pantelidou K, Karamitsos D, et al: Prevalence of rheumatic diseases in Greece: a cross-sectional population based epidemiological study. The ESORDIG Study. J Rheumatol 2003, 30:1589-1601

42. Biino G, Casula L, de TF, Adamo M, Vaccargiu S, Francavilla M, Loi D, Casti A, Atzori M, Pirastu M: Epidemiology of osteoporosis in an isolated Sardinian population by using quantitative ultrasound. Am J Epidemiol 2011, 174:432-439.

43. Bleicher K, Naganathan V, Cumming RG, Seibel MJ, Sambrook PN, Blyth FM, Le Couteur DG, Handelsman DJ, Waite LM, Creasey HM: Prevalence and treatment of osteoporosis in older Australian men: findings from the CHAMP study. Med J Aust 2010, 193:387-391.

44. Cui LH, Choi JS, Shin MH, Kweon SS, Park KS, Lee YH, Nam HS, Jeong SK, Im $J S$ : Prevalence of osteoporosis and reference data for lumbar spine and hip bone mineral density in a Korean population. J Bone Miner Metab 2008, 26:609-617.

45. Henry MJ, Pasco JA, Nicholson GC, Seeman E, Kotowicz MA: Prevalence of osteoporosis in Australian women: Geelong Osteoporosis Study. J Clin Densitom 2000, 3:261-268.

46. Holt G, Khaw KT, Reid DM, Compston JE, Bhalla A, Woolf AD, Crabtree NJ, Dalzell N, Wardley-Smith B, Lunt M, et al: Prevalence of osteoporotic bone mineral density at the hip in Britain differs substantially from the US over 50 years of age: implications for clinical densitometry. BrJ Radiol 2002, 75:736-742.

47. Kenny AM, Smith J, Noteroglu E, Waynik IY, Ellis C, Kleppinger A, Annis K, Dauser D, Walsh S: Osteoporosis risk in frail older adults in assisted living. J Am Geriatr Soc 2009, 57:76-81.

48. Naves M, Diaz-Lopez JB, Gomez C, Rodriguez-Rebollar A, Serrano-Arias M, Cannata-Andia JB: Prevalence of osteoporosis in men and determinants of changes in bone mass in a non-selected Spanish population. Osteoporos Int 2005, 16:603-609.

49. Sanfelix-Genoves J, Reig-Molla B, Sanfelix-Gimeno G, Peiro S, Graells-Ferrer M, Vega-Martinez M, Giner V: The population-based prevalence of osteoporotic vertebral fracture and densitometric osteoporosis in postmenopausal women over 50 in Valencia, Spain (the FRAVO study). Bone 2010, 47:610-616.
50. Shin CS, Choi HJ, Kim MJ, Kim JT, Yu SH, Koo BK, Cho HY, Cho SW, Kim SW, Park YJ, et al: Prevalence and risk factors of osteoporosis in Korea: a community-based cohort study with lumbar spine and hip bone mineral density. Bone 2010, 47:378-387.

51. Yang TS, Chen YR, Chen YJ, Chang CY, Ng HT: Osteoporosis: prevalence in Taiwanese women. Osteoporos Int 2004, 15:345-347.

52. Vestergaard P, Rejnmark L, Mosekilde L: Osteoporosis is markedly underdiagnosed: a nationwide study from Denmark. Osteoporos Int 2005, 16:134-141.

53. Cheng H, Gary LC, Curtis JR, Saag KG, Kilgore ML, Morrisey MA, Matthews R, Smith W, Yun H, Delzell E: Estimated prevalence and patterns of presumed osteoporosis among older Americans based on Medicare data. Osteoporos Int 2009, 20:1507-1515.

54. Kotz K, Deleger S, Cohen R, Kamigaki A, Kurata J: Osteoporosis and health-related quality-of-life outcomes in the Alameda County Study population. Prev Chronic Dis 2004, 1:A05

55. Lespessailles E, Cotte FE, Roux C, Fardellone P, Mercier F, Gaudin AF: Prevalence and features of osteoporosis in the French general population: the Instant study. Joint Bone Spine 2009, 76:394-400.

56. Saks K, Kolk H, Allev R, Soots A, Koiv K, Paju I, Jaanson K, Schneider G: Health status of the older population in Estonia. Croat Med J 2001, 42:663-668.

57. Werner P: Self-reported prevalence and correlates of osteoporosis: results from a representative study in Israel. Arch Gerontol Geriatr 2003, 37:277-292

58. World Health Organization: Assessment of fracture risk and its application to screening for postmenopausal osteoporosis. Geneva: 1994.

59. Baek SR, Lim JY, Lim JY, Park JH, Lee JJ, Lee SB, Kim KW, Paik NJ: Prevalence of musculoskeletal pain in an elderly Korean population: results from the Korean Longitudinal Study on Health and Aging (KLoSHA). Arch Gerontol Geriatr 2010, 51:e46-e51.

60. Cecchi F, Debolini P, Lova RM, Macchi C, Bandinelli S, Bartali B, Lauretani F, Benvenuti $E_{1}$ Hicks G, Ferrucci L: Epidemiology of back pain in a representative cohort of Italian persons 65 years of age and older: the InCHIANTI study. Spine (Phila Pa 1976) 2006, 31:1149-1155.

61. Chiu TT, Leung AS: Neck pain in Hong Kong: a telephone survey on prevalence, consequences, and risk groups. Spine (Phila Pa 1976) 2006, 31:E540-E544

62. Denard PJ, Holton KF, Miller J, Fink HA, Kado DM, Marshall LM, Yoo JU: Back pain, neurogenic symptoms, and physical function in relation to spondylolisthesis among elderly men. Spine J 2010, 10:865-873.

63. Docking RE, Fleming J, Brayne C, Zhao J, Macfarlane GJ, Jones GT: Epidemiology of back pain in older adults: prevalence and risk factors for back pain onset. Rheumatology (Oxford) 2011, 50:1645-1653.

64. Freburger JK, Holmes GM, Agans RP, Jackman AM, Darter JD, Wallace AS, Castel LD, Kalsbeek WD, Carey TS: The rising prevalence of chronic low back pain. Arch Intern Med 2009, 169:251-258.

65. Goode AP, Freburger J, Carey T: Prevalence, practice patterns, and evidence for chronic neck pain. Arthritis Care Res (Hoboken) 2010, 62:1594-1601.

66. Goubert L, Crombez G, De B: I: Low back pain, disability and back pain myths in a community sample: prevalence and interrelationships. Eur J Pain 2004, 8:385-394.

67. Guez M, Hildingsson C, Nilsson M, Toolanen G: The prevalence of neck pain: a population-based study from northern Sweden. Acta Orthop Scand 2002, 73:455-459.

68. Hartvigsen J, Christensen K, Frederiksen H: Back and neck pain exhibit many common features in old age: a population-based study of 4,486 Danish twins 70-102 years of age. Spine (Phila Pa 1976) 2004, 29:576-580

69. Hartvigsen J, Frederiksen $\mathrm{H}$, Christensen K: Back and neck pain in seniorsprevalence and impact. Eur Spine J 2006, 15:802-806.

70. Hartvigsen J, Christensen K: Pain in the back and neck are with us until the end: a nationwide interview-based survey of Danish 100-year-olds. Spine (Phila Pa 1976) 2008, 33:909-913.

71. Hicks GE, Gaines JM, Shardell M, Simonsick EM: Associations of back and leg pain with health status and functional capacity of older adults: findings from the retirement community back pain study. Arthritis Rheum 2008, 59:1306-1313.

72. Jacobs JM, Hammerman-Rozenberg R, Cohen A, Stessman J: Chronic back pain among the elderly: prevalence, associations, and predictors. Spine (Phila Pa 1976) 2006, 31:E203-E207. 
73. Keenan AM, Tennant A, Fear J, Emery P, Conaghan PG: Impact of multiple joint problems on daily living tasks in people in the community over age fifty-five. Arthritis Rheum 2006, 55:757-764.

74. Meyer T, Cooper J, Raspe H: Disabling low back pain and depressive symptoms in the community-dwelling elderly: a prospective study. Spine (Phila Pa 1976) 2007, 32:2380-2386.

75. Miro J, Paredes S, Rull M, Queral R, Miralles R, Nieto R, Huguet A, Baos J: Pain in older adults: a prevalence study in the Mediterranean region of Catalonia. Eur J Pain 2007, 11:83-92.

76. Natvig B, Ihlebaek C, Grotle M, Brage S, Bruusgaard D: Neck pain is often a part of widespread pain and is associated with reduced functioning. Spine (Phila Pa 1976) 2010, 35:E1285-E1289.

77. Parsons S, Breen A, Foster NE, Letley L, Pincus T, Vogel S, Underwood M: Prevalence and comparative troublesomeness by age of musculoskeletal pain in different body locations. Fam Pract 2007, 24:308-316.

78. Picavet HS, Hazes JM: Prevalence of self reported musculoskeletal diseases is high. Ann Rheum Dis 2003, 62:644-650

79. Salaffi F, De AR, Grassi W: Prevalence of musculoskeletal conditions in an Italian population sample: results of a regional community-based study. 1. The MAPPING study. Clin Exp Rheumatol 2005, 23:819-828.

80. Santos-Eggimann B, Wietlisbach V, Rickenbach M, Paccaud F, Gutzwiller F: One-year prevalence of low back pain in two Swiss regions: estimates from the population participating in the 1992-1993 MONICA project. Spine (Phila Pa 1976) 2000, 25:2473-2479.

81. Stranjalis G, Tsamandouraki K, Sakas DE, Alamanos Y: Low back pain in a representative sample of Greek population: analysis according to personal and socioeconomic characteristics. Spine (Phila Pa 1976) 2004, 29:1355-1360.

82. Strine TW, Hootman JM: US national prevalence and correlates of low back and neck pain among adults. Arthritis Rheum 2007, 57:656-665.

83. Suka M, Yoshida K: The national burden of musculoskeletal pain in Japan: Projections to the year 2055. Clin J Pain 2009, 25:313-319.

84. Vogt MT, Simonsick EM, Harris TB, Nevitt MC, Kang JD, Rubin SM, Kritchevsky SB, Newman AB: Neck and shoulder pain in 70- to 79-year-old men and women: findings from the Health, Aging and Body Composition Study. Spine J 2003, 3:435-441.

85. Webb R, Brammah T, Lunt M, Urwin M, Allison T, Symmons D: Prevalence and predictors of intense, chronic, and disabling neck and back pain in the UK general population. Spine (Phila Pa 1976) 2003, 28:1195-1202.

86. Yaron M, Caspi D, Kaufman I, Zemach M, Grotto I, Altman R: Estimation of the prevalence of rheumatic diseases in Israel. Semin Arthritis Rheum 2011, 40:473-478.

87. Thomas E, Peat G, Harris L, Wilkie R, Croft PR: The prevalence of pain and pain interference in a general population of older adults: cross-sectional findings from the North Staffordshire Osteoarthritis Project (NorStOP). Pain 2004, 110:361-368.

88. Hill CL, Gill TK, Shanahan EM, Taylor AW: Prevalence and correlates of shoulder pain and stiffness in a population-based study: the North West Adelaide Health Study. Int J Rheum Dis 2010, 13:215-222.

89. Gummesson C, Atroshi I, Ekdahl C, Johnsson R, Ornstein E: Chronic upper extremity pain and co-occurring symptoms in a general population. Arthritis Rheum 2003, 49:697-702.

90. Christmas C, Crespo CJ, Franckowiak SC, Bathon JM, Bartlett SJ, Andersen RE: How common is hip pain among older adults? Results from the Third National Health and Nutrition Examination Survey. J Fam Pract 2002, 51:345-348.

91. Dawson J, Linsell L, Zondervan K, Rose P, Randall T, Carr A, Fitzpatrick R: Epidemiology of hip and knee pain and its impact on overall health status in older adults. Rheumatology (Oxford) 2004, 43:497-504.

92. Peat $G$, Thomas E, Wilkie R, Croft P: Multiple joint pain and lower extremity disability in middle and old age. Disabil Rehabil 2006, 28:1543-1549.

93. Croft P, Jordan K, Jinks C: "Pain elsewhere" and the impact of knee pain in older people. Arthritis Rheum 2005, 52:2350-2354.

94. Jinks C, Jordan KP, Blagojevic M, Croft P: Predictors of onset and progression of knee pain in adults living in the community. A prospective study. Rheumatology (Oxford) 2008, 47:368-374.

95. Badlissi F, Dunn JE, Link CL, Keysor JJ, McKinlay JB, Felson DT: Foot musculoskeletal disorders, pain, and foot-related functional limitation in older persons. J Am Geriatr Soc 2005, 53:1029-1033.
96. Menz HB, Morris ME: Footwear characteristics and foot problems in older people. Gerontology 2005, 51:346-351.

97. Mickle KJ, Munro BJ, Lord SR, Menz HB, Steele JR: Foot pain, plantar pressures, and falls in older people: a prospective study. J Am Geriatr Soc 2010, 58:1936-1940.

98. Molgaard C, Lundbye-Christensen S, Simonsen O: High prevalence of foot problems in the Danish population: a survey of causes and associations. Foot (Edinb) 2010, 20:7-11.

99. Dunn JE, Link CL, Felson DT, Crincoli MG, Keysor JJ, McKinlay JB: Prevalence of foot and ankle conditions in a multiethnic community sample of older adults. Am J Epidemio/ 2004, 159:491-498.

100. Natvig B, Bruusgaard D, Eriksen W: Localized low back pain and low back pain as part of widespread musculoskeletal pain: two different disorders? A cross-sectional population study. J Rehabil Med 2001, $33: 21-25$.

101. Leveille SG, Zhang Y, McMullen W, Kelly-Hayes M, Felson DT: Sex differences in musculoskeletal pain in older adults. Pain 2005 , 116:332-338.

102. Fillingim RB, King CD, Ribeiro-Dasilva MC, Rahim-Williams B, Riley JL III: Sex gender, and pain: a review of recent clinical and experimental findings. J Pain 2009, 10:447-485.

103. Exton-Smith AN: Epidemiological studies in the elderly: methodological considerations. Am J Clin Nutr 1982, 35:1273-1279.

104. Sjogren P, Ekholm O, Peuckmann V, Gronbaek M: Epidemiology of chronic pain in Denmark: an update. Eur J Pain 2009, 13:287-292.

105. Pickering G, Jourdan D, Eschalier A, Dubray C: Impact of age, gender and cognitive functioning on pain perception. Gerontology 2002, 48:112-118

106. Macfarlane GJ, Jones GT, Knekt P, Aromaa A, McBeth J, Mikkelsson M, Heliovaara M: Is the report of widespread body pain associated with long-term increased mortality? Data from the Mini-Finland Health Survey. Rheumatology (Oxford) 2007, 46:805-807

107. Kroot EJ, van Leeuwen MA, van Rijswijk MH, Prevoo ML, Hof MA V't, van De Putte LB, van Riel PL: No increased mortality in patients with rheumatoid arthritis: up to 10 years of follow up from disease onset. Ann Rheum Dis 2000, 59:954-958.

108. Kulminski AM, Kulminskaya IV, Ukraintseva SV, Land K, Yashin Al: An inverse association between self-reported arthritis and mortality in the elderly: findings from the national long-term care survey. Rejuvenation Res 2008, 11:251-257.

109. Bressler HB, Keyes WJ, Rochon PA, Badley E: The prevalence of low back pain in the elderly. A systematic review of the literature. Spine (Phila Pa 1976) 1999, 24:1813-1819.

110. Dionne CE, Dunn KM, Croft PR: Does back pain prevalence really decrease with increasing age? A systematic review. Age Ageing 2006, 35:229-234.

111. Luime JJ, Koes BW, Hendriksen IJ, Burdorf A, Verhagen AP, Miedema HS, Verhaar JA: Prevalence and incidence of shoulder pain in the general population; a systematic review. Scand J Rheumatol 2004, 33:73-81.

112. Dagenais S, Garbedian S, Wai EK: Systematic review of the prevalence of radiographic primary hip osteoarthritis. Clin Orthop Relat Res 2009, 467:623-637.

113. Kuorinka I, Jonsson B, Kilbom A, Vinterberg H, Sorensen FB, Andersson G, Jorgensen K: Standardised Nordic Questionnaires for the Analysis of Musculoskeletal Symptoms. App/ Ergon 1987, 18:233-237.

114. Edwards P, Clarke M, Diguiseppi C, Pratap S, Roberts I, Wentz R: Identification of randomized controlled trials in systematic reviews: accuracy and reliability of screening records. Stat Med 2002, 21:1635-1640

115. Hartling L, Ospina M, Liang Y, Dryden DM, Hooton N, Krebs SJ, Klassen TP: Risk of bias versus quality assessment of randomised controlled trials: cross sectional study. BMJ 2009, 339:b4012.

doi:10.1186/2045-709X-20-31

Cite this article as: Fejer and Ruhe: What is the prevalence of musculoskeletal problems in the elderly population in developed countries? A systematic critical literature review. Chiropractic \& Manual Therapies 2012 20:31 